\title{
Functional analysis of the CDPK-Related Kinase (CRK) family in Arabidopsis thaliana
}

\author{
PhD thesis
}

Abu Imran Baba

Supervisors:

Dr. Ágnes Cséplő

Dr. Gábor Rigó

Laboratory of Arabidopsis Molecular Genetics Institute of Plant Biology

Biological Research Centre of the Hungarian Academy of Sciences

Doctoral School of Biology, Faculty of Science and Informatics University of Szeged

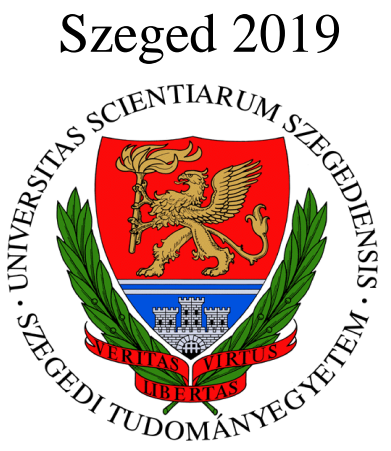




\section{Table of Contents}

LIST OF ABBREVIATIONS .............................................................................. 1

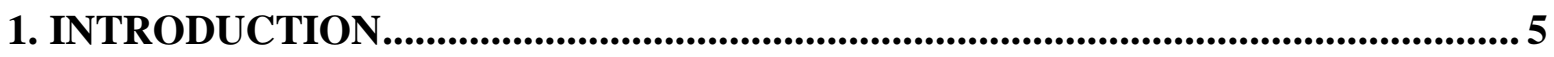

1.1. Arabidopsis thaliana, as a dicot genetical model plant .................................................. 5

1.2. Calcium-Dependent Protein Kinase (CDPKs) superfamily .................................. 6

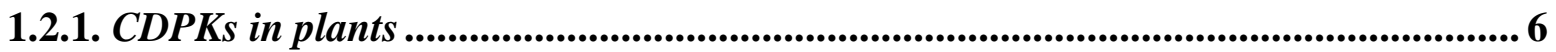

1.2.2. CDPK Related Kinase (CRK) subfamily ....................................................8

1.3. Auxin transport and its signaling ........................................................................... 10

1.3.1. Auxin an important hormone in plant development ........................................... 10

1.3.2. Mechanism of directional auxin transport ........................................................ 11

1.3.3. Auxin transporters ................................................................................................. 12

1.3.3.1. Auxin efflux PIN transporters ......................................................................... 14

1.3.3.2. Auxin influx protein transporters .................................................................... 15

1.3.3.3. Polar localization and trafficking of PINs ....................................................... 16

1.3.4. Embryogenesis in Arabidopsis and the role of auxin transporters ..................... 18

1.3.4.1. Embryo development and its specification .................................................... 18

1.3.4.2. Expression and polarity of auxin infux/efflux transporters in embryos .............. 19

1.3.5. Auxin signaling................................................................................................... 20

1.3.6. Hormonal crosstalk during embryogenesis .............................................................. 22

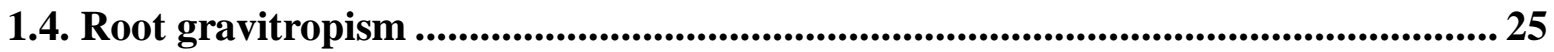

1.5. Photosynthesis and light-induced damage in plants ................................................. 27

1.5.1. Reactive oxygen species $(\mathrm{ROS})$................................................................. 27

1.5.2. Singlet oxygen $\left({ }^{1} \mathrm{O}_{2}\right)$ as one of the potent $\mathrm{ROS}$ species in cell death ...................... 28

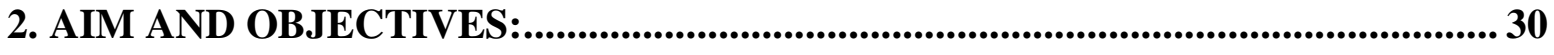

3. MATERIALS AND METHODS: .......................................................................... 31

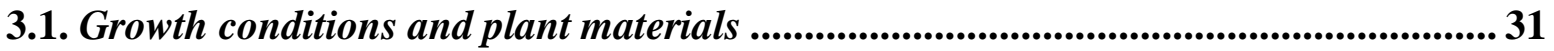

3.1.1. Seed/embryo size determination and embryo isolation ........................................ 31

3.2. Genotyping and transcript analysis of the T-DNA insertion mutants .................... 32

3.3. Phenotyping of the T-DNA insertion mutants ...................................................... 32

3.3.1. Germination and root growth assay ............................................................................. 32

3.3.2. Root and hypocotyl gravitropic bending assay ........................................................ 33

3.3.3. Fresh weight, chlorophyll and carotenoid content measurement .......................... 33 
3.4. Plant transformation and GFP localization studies ................................................ 33

3.4.1. Gene cloning and plant transformation ......................................................... 33

3.4.2. Protoplast transformation studies................................................................ 34

3.4.3. Hairy root transformation for the 35S::cCRK1-GFP ..................................... 35

3.5. Physiological characterization of the Arabidopsis CRKs.................................... 35

3.5.1. PSII photochemical activity measurements ............................................................. 35

3.5.2. Detection of ${ }^{1} \mathrm{O}_{2}$ production ........................................................................ 36

3.5.3. Determination of cell death .................................................................................................. 36

3.6. Microsomal membrane preparation, differential centrifugation and

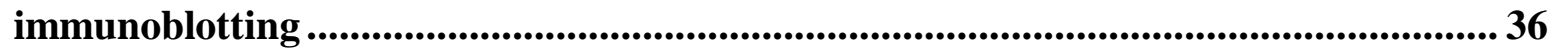

3.7. RNA isolation and quantitative real time PCR (qRT-PCR).................................. 38

3.7.1. RNA isolation and $q R T-P C R$ from seedlings ..................................................... 38

3.7.2. RNA isolation and $q R T-P C R$ from embryos............................................................... 38

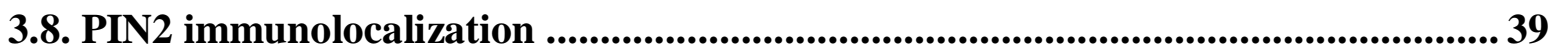

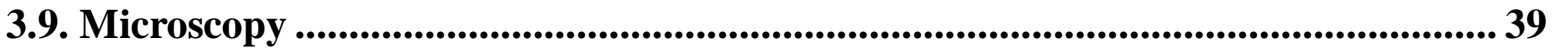

3.9.1. Intracellular GFP localization of roots by confocal laser scanning microscope .... 39

3.9.2. Abundance of GFP signal in embryos by LSM microscopy ................................. 39

3.9.3. Embryo morphology monitoring by Cell-R microscope ...................................... 40

3.10. Total gibberellic acid (GA) measurement by Competitive GA ELISA assay ...... 40

3.11. Cloning of the PIN4 and PIN7 hydrophilic loop (HL) ................................... 40

3.12. Protein expression and purification .................................................................... 41

3.13. In vitro kinase assay .................................................................................................. 42

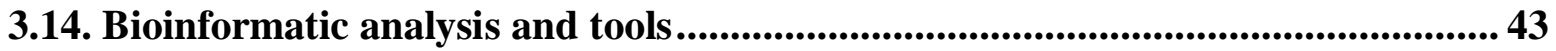

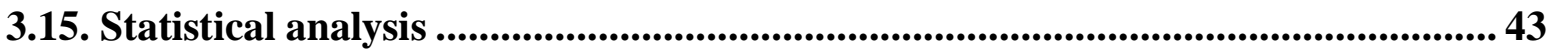

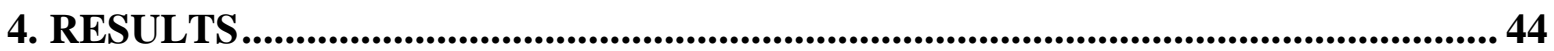

4.1. Phylogenetic analysis of CDPK-Related Kinase (AtCRK) family in Arabidopsis

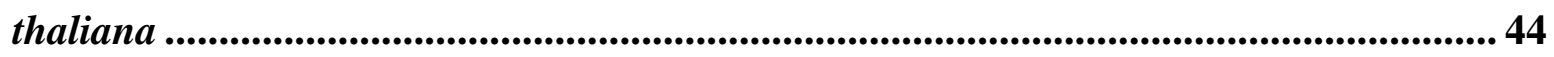

4.2. Expression analysis of AtCRK genes in variable light conditions ............................ 44

4.3. Intracellular protein localization of AtCRK protein kinase family ........................ 45

4.3.1. Protoplast transient expression assay ................................................................................ 46

4.3.2. In planta subcellular localization 35S::cCRK-eGFP fusion proteins..................... 46

4.3.3. Differential centrifugation and western hybridization of AtCRK-eGFPs.............. 50

4.4. Functional analysis of the AtCRK genes...............................................................51

4.4.1. T-DNA insertion mutants of AtCRK family ....................................................... 51 
4.4.2. Expression levels of Atcrk mutants 52

4.5. Phenotypic characterization of the Atcrk mutants ..........................................5 53

4.6. Immunolocalization of PIN2 in roots of Atcrk mutants ........................................5 56

4.7. Light sensitive phenotype of Atcrk1-1 T-DNA insertion mutant..............................57

4.8. Singlet oxygen $\left({ }^{1} \mathrm{O}_{2}\right)$ accumulation and cell death in Atcrk1-1 mutant ................... 60

4.9. Role of AtCRK5 protein kinase in embryogenesis of Arabidopsis thaliana............ 62

4.9.1. Decreased seed size and delayed embryo bending phenotype in Atcrk5-1 ............. 62

4.9.2. Delayed embryo development and morphological changes in the late Atcrk5-1 mutant embryos 63

4.9.3. Role of gibberellic acid and auxin cross talk in the development of embryos in Atcrk5-1 mutant ...................................................................................................................66 66

4.9.4. Distribution of auxin and its transporters in the Atcrk5-1 mutant ......................... 70

5. DISCUSSION ........................................................................................................ 78

5.1. Functional analysis and subcellular localization of AtCRK protein kinase family... 78

5.2. Sensitivity of Atcrk1-1 mutant under continuous illumination ...............................80

5.3. Role of AtCRK5 protein kinase in embryo development ........................................... 82

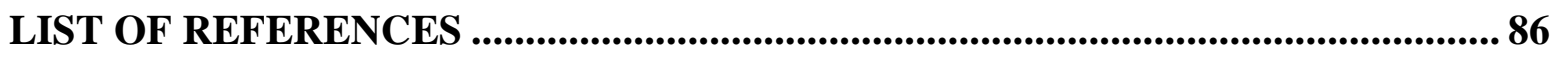

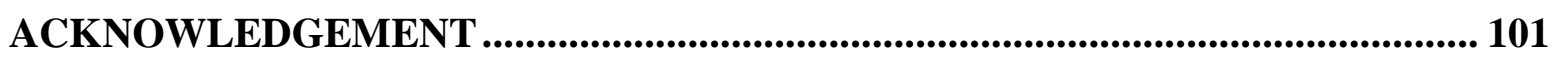

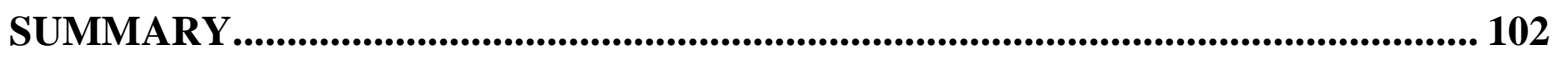

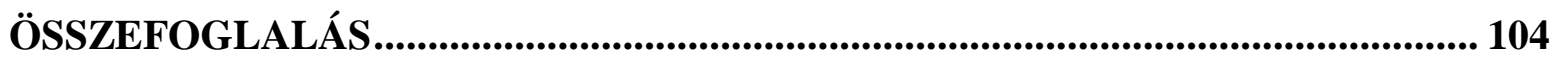

PUBLICATION LIST (MTMT ID: 10053163)................................................... 107

SUPPLEMENTARY DATA ................................................................................. 109 


\section{LIST OF FIGURES}

Figure 1: Domain structure of CDPKs, CRKs and two other related kinases.

Figure 2: Chemical structure of auxin.

Figure 3: Chemiosmosis hypothesis for polar auxin transport.

Figure 4: The predicted structure of PIN protein.

Figure 5: Polar localization of PIN proteins in the Arabidopsis root tip.

Figure 6: Formation of apical-basal polarity during Arabidopsis embryogenesis.

Figure 7: Components in auxin perception and signaling.

Figure 8: Schematic trend of hormone accumulation during seed development.

Figure 9: Auxin transport during root gravitropic response in root.

Figure 10: Schematic map of 35S::cCRKs-GFP vector construct.

Figure11: Phylogenetic tree of AtCRK proteins.

Figure12: AtCRK gene expression in wild type Arabidopsis plants.

Figure 13: Sequence Alignment of N-terminal myristoylation/palmitoylation.

Figure 14: Transient expression of pCaMV35S::CRKs-GFP in Arabidopsis protoplasts.

Figure 15: Intracellular localization of AtCRK1-eGFP fusion protein.

Figure 16: Intracellular localization of the AtCRK1-eGFP fusion protein in dark grown seedlings.

Figure 17: Intracellular localization of the AtCRK2-eGFP, AtCRK3-eGFP and AtCRK4-eGFP proteins.

Figure 18: Intracellular localization of the AtCRK5-eGFP, AtCRK7-eGFP and AtCRK8-eGFP proteins.

Figure 19: Distribution of AtCRK-eGFP proteins in subcellular fractions.

Figure 20: Schematic map of the gene with positions of the T-DNA insertions of various Atcrk mutants.

Figure 21: Transcript levels of the Atcrk mutants.

Figure 22: Germination assay of Atcrk mutants.

Figure 23: Relative root growth of Atcrk mutants.

Figure 24: Root gravitropic test.

Figure 25: Hypocotyl gravitropic test.

Figure 26: Immunolocalization of PIN2 in wild type Col-0 and the Atcrk mutants.

Figure 27: Light sensitivity of the Atcrk1-1 mutant.

Figure 28: Fresh weight and chlorophyll $a+b$ contents of three weeks old plantlets.

Figure 29: Carotenoid, Chlorophyll $b$ and Chlorophyll $a$ contents of three weeks old Atcrk $1-1$ plantlets.

Figure 30: Photosynthetic parameters of Atcrk1-1 mutant under continuous light.

Figure 31: ROS accumulation in Atcrk1-1 mutant. 
Figure 32: Cell death in Atcrk1-1 mutant.

Figure 33: Seed and embryo phenotype of wild type (Col-0) and mutant (Atcrk5-1).

Figure 34: Embryo developmental stages in Atcrk5-1 mutant compared with wild type Col-0.

Figure 35: Quantitative analysis of embryogenesis in wild type Arabidopsis thaliana (Col-0) and Atcrk5-1 mutant correlated to siliques development.

Figure 36: Comparison of SAM-RAM axis size in bent cotyledon embryos within the siliques derived seeds after precleared in Hoyer's solution.

Figure 37: Role of gibberellic acid in development of embryo.

Figure 38: Expression of various genes during embryo development.

Figure 39: DR5::GFP as an auxin sensor for measurement of auxin levels in wild type Arabidopsis and mutant Atcrk5-1 embryos at various developmental stages.

Figure 40: Abundance of PIN1-GFP transporter in wild type and in Atcrk5-1 mutant embryos during embryogenesis.

Figure 41: Abundance of the PIN7-GFP transporter in Arabidopsis embryos at various developmental stages.

Figure 42: Abundance of PIN4-GFP transporter in wild type Arabidopsis and mutant Atcrk5-1 embryos at several developmental stages.

Figure 43: Expression of AUX1::YFP from heart till walking stick stages of embryo development.

Figure 44: Analysis of the purified MBP-PIN4HL and MBP-PIN7HL proteins.

Figure 45: Non-radioactive kinase assay.

Figure 46: The identified CRK5 specific phospho-peptide on MBP-PIN4HL and MBP-PIN7HL.

Figure S1: Primer pairs used in this overall study designed by Primer3plus software.

Figure S2: Genomic region of PIN7 containing a small intron sequence.

Figure S3: Comparison of expression studies for public transcript analysis profiling data from eFP Browser.

Figure S4: Expression data from a high resolution map of Arabidopsis thaliana developmental transcriptome AtCRK1 and AtCRK5 based on RNA-seq profiling from eFP Browser.

Figure S5: Subcellular protein localization database of AtCRK proteins.

Figure S6: Ruling out the mitochondrial specific marker localization for AtCRK1-eGFP.

Figure S7: Ruling out the Golgi specific m-cherry marker localization of AtCRK1-eGFP.

Figure S8: Photosynthetic activities of five Atcrk mutants in continuous light conditions.

Figure S9: Heat maps of the PIN1-GFP (A) in Col-0 and (B) Atcrk5-1 mutant.

Figure S10: PIN3-GFP abundance during several stages of embryo development.

Figure S11: Localization of the AtCRK5-GFP in embryos at several developmental stages. 


\section{LIST OF ABBREVIATIONS}

1-NAA 1-Naphthylacetic acid

${ }^{1} \mathrm{O}_{2} \quad$ Singlet oxygen

2,4-D 2,4-dichlorophenoxyacetic acid

4-Cl-IAA 4-chloroindole-3-acetic acid

ABCB ATP-binding cassette family B

ABI3

ABSCISIC ACID INSENSITIVE 3

ABP

Auxin binding protein 1

ARF

Auxin response factor

Arqua-1

Agrobacterium rhizogenes strain-1

AUX1

AUXIN-RESISTANT 1

BFA

Brefeldin A

CaM Calmodulin

CaMV35S Cauliflower Mosaic Virus 35S promoter

CCaMKs Calcium and Calmodulin-dependent protein kinases

cDNA Complementary DNA

CDPK Calcium Dependent Protein Kinases

Chl Chlorina

CIB5 Cryptochrome-interacting basic helix-loop-helix5

CL Continuous light

CLSM Confocal Laser Scanning Microscope

Col-0 Arabidopsis wild type, Columbia ecotype

CRK CDPK Related Kinase

CTL1 Choline transporter like 1

Cy Cytosolic

DAP Days after pollination

DTT Dithiothreitol

EDTA Ethylenediaminetetraacetic acid

ELISA Enzyme-linked immunosorbent assay 
ERF 13 Ethylene response factor 13

ERF Ethylene response factor

ETR Electron transport rate

Fm Maximum fluorescence in dark

Fv/Fm Maximum quantum yield

GA Gibberellic acid

GA20ox Gibberellin 20-oxidase

GA20x Gibberellin 2-oxidase

GA3ox Gibberellin 3-oxidase

GAI GA INSENSITIVE

GAPDH2 Glyceraldehyde-3-phosphate dehydrogenase

GFP Green fluorescent protein

$\mathbf{H}_{2} \mathrm{O}_{2} \quad$ Hydrogen peroxide

HRP Horseradish peroxidase

HSE Heat-shock element

HSF Heat shock transcription factor

IAA Indole-3-acetic acid

IBA Indole-3-butyric acid

IPTG Isopropyl $\beta$-D-1-thiogalactopyranoside

KD Knock-down

KO Knock-out

LAX Like AUX

LB Left border

LD Laser diode

LEC1 LEAFY COTYLEDON1

MDA Malondialdehyde

Mi Microsomal

MP-BDL MONOPTEROS-BODENLOS

MS Murashige and Skoog medium

NaCl Sodium chloride 


$\begin{array}{ll}\text { Nm } & \text { Nanometer } \\ \text { NPQ } & \text { Non photochemical quenching } \\ \text { OD } & \text { Optical density } \\ \text { P- Value } & \text { Probability value } \\ \text { PAM } & \text { Pulse amplitude modulation } \\ \text { PAR } & \text { Photosynthetic active radiation } \\ \text { PAT } & \text { Polar auxin transport } \\ \text { Pchlide } & \text { Protochlorophyllide } \\ \text { PEG } & \text { Polyethylene glycol } \\ \text { PEPRKS } & \text { Phosphoenolpyruvate carboxylase kinase-related kinases } \\ \text { PGP } & \text { P-glycoproteins } \\ \text { PIC } & \text { Protease inhibitor cocktail } \\ \text { PILS } & \text { PIN-like transporters } \\ \text { PIN } & \text { PIN FORMED } \\ \text { pK7FWG2.0 } & \text { GATEWAY based CaMV 35S promoter vector } \\ \text { PM } & \text { Plasma membrane } \\ \text { PMSF } & \text { Phenylmethylsulfonyl fluoride } \\ \text { PPCK } & \text { Phosphoenolpyruvate carboxylase kinases } \\ \text { PS } & \text { Photosystem } \\ \text { PVDF } & \text { Polyvinylidene fluoride } \\ \text { qRT-PCR } & \text { Real-time quantitative polymerase chain reaction } \\ \text { RAM } & \text { Root apical meristem } \\ \text { RB } & \text { Right border } \\ \text { RGA } & \text { REPRESSOR OF gal-3 } \\ \text { RGL } & \text { REPRESSOR OF gal-3-LIKE protein } \\ \text { ROS } & \text { Reactive oxygen species } \\ \text { SALK } & \text { Salk Institute Genomic Analysis Laboratory } \\ \text { SAM } & \text { Shoot apical meristem } \\ \text { SCF } & \text { Skp1-cullin-Fbox protein } \\ \text { SD } & \text { Short-day } \\ & \\ \text { PAM } & \end{array}$


SDS-PAGE Sodium dodecyl sulfate polyacrylamide gel electrophoresis

SE Standard error

SnRKs Sucrose non-fermenting-1 (SNF1)-related protein kinases

SOSG Singlet oxygen sensor green

T1 Fist generation

T3 Third generation

TBB $\quad \beta$-tubulin

TBST Tris Buffered Saline with Tween

T-DNA Transfer DNA

TF Transcription factor

TIR1 Transport Inhibitor Response 1

TPL TOPLESS

WAT1 WALLS ARE THIN 1

WRKY Zinc finger transcription factor

Y (NO) Non-regulated non photochemical quenching

YFP Yellow fluorescent protein

$\boldsymbol{\Phi}_{\text {PSII }} \quad$ Effective PSII quantum yield 


\section{INTRODUCTION}

\subsection{Arabidopsis thaliana, as a dicot genetical model plant}

Arabidopsis thaliana is a medium-sized flowering plant of the mustard family (Cruciferae or Brassicaceae) distributed throughout Europe, Asia, and North America (Meyerowitz and Somerville 1994). It is an important plant of dicotyledonous weed species from Brassicaceae family, relatives of the economically important crop plants like turnip, cabbage and broccoli. Many different ecotypes have been collected from natural populations and are available for experimental analysis (David et al., 1998; Weigel, 2012). The entire life cycle, including seed germination, formation of a rosette plant, flowering and maturation of the first seeds, is completed in six weeks (David et al., 1998). Almost everything in this plant, ranging from its genomic size to its life cycle is short. One of the most widespread natural varieties of Arabidopsis thaliana is Columbia ecotype (Col-0). Arabidopsis is associated with classical genetic research and it has been in the focus of intense genetic, biochemical and physiological study for many years due to its several desirable laboratory traits as a model plant. Full genomic sequence analysis of Arabidopsis thaliana 5 pairs of chromosomes was completed in the year 2000 (The Arabidopsis Genome Initiative, 2000).

Arabidopsis has a fast life cycle; it is self-compatible and produces increased yield of seeds per generation. It has a small genome of approximately 135 megabase pairs (Mbp) and chromosome number $(2 \mathrm{n}=10)$. Information obtained from Arabidopsis genetical and molecular works can be exploited for other dicot gene functional analysis in the near future. Thousands of Arabidopsis T-DNA insertion mutants have been created so far and the induction of such mutations greatly facilitates the investigation of the functions of various genes in Arabidopsis (Koncz et al., 1992; Alvarado et al., 2004; Coego et al., 2014). The complete sequencing of Arabidopsis genome lead to the identification of all the protein kinases present in a model plant. The Arabidopsis genome encodes 1,085 typical protein kinases which constitute about $4 \%$ of the predicted 25,500 genes (Hrabak et al., 2003). The data from the functional analysis of Arabidopsis thaliana genes can be used to study the corresponding genes of other dicotyledonous plants, which in the near future can greatly facilitate plant nutrition and research. 


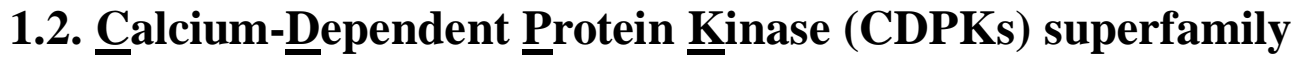 \\ 1.2.1. CDPKs in plants}

The calcium-dependent protein kinase $(\mathrm{CDPK})$ is a Serine/Threonine protein kinase that plays vital roles in plant growth, development, and responses to multiple stresses. CDPKs also named CPKs, have been identified throughout the plant kingdom as well as in several protists, but are absent in animals (Harper and Harmon, 2005). CDPKs constitute one of the largest subfamilies of plant protein kinase family. They possess a characteristic structure with an N-terminal Ser/Thr protein kinase domain fused to a carboxy-terminal calmodulin-like domain containing EF-hand calcium-binding sites (Harmon et al., 2000; Cheng et al., 2002) (Figure 1). In plants, the multigene family of CDPKs have been shown to encode structurally conserved, unimolecular calcium sensor/protein kinase effector proteins and CDPKs are ideal kinase proteins for perceiving intracellular changes in $\mathrm{Ca}^{2+}$ concentration and translating them into specific phosphorylation events to initiate further downstream signaling cascade (Schulz et al., 2013; Simeunovic et al., 2016). Therefore, CDPKs do not depend on the interaction with exogenous calmodulin but can be activated by $\mathrm{Ca}^{2+}$ binding directly. A junction domain between the kinase and calmodulin-like domain functions as a pseudo-substrate autoinhibitor that inhibits phosphorylation in the absence of $\mathrm{Ca}^{2+}$ and keeps the CDPK in a state of low activity (Harmon et al., 1994).

The genome analysis of Arabidopsis thaliana identified 34 calcium-dependent protein kinase (CDPKs), eight CDPK-related kinases (CRKs), two PPCKs, two phosphoenolpyruvate carboxylase kinases (PEPRKs), and 38 Sucrose non-fermenting-1 (SNF1)-related protein kinases (SnRKs) (Hrabak et al., 2003). CDPKs are present in plants and a specific subgroup of protists, but CRKs, PPCKs, PEPRKs, and two of the SnRK subgroups have been found only in plants (Hrabak et al., 2003). The majority of CDPKs have predicted myristoylation sites at their $\mathrm{N}$ terminal assuming that these proteins are membrane localized, and these CDPKs also have a nearby Cys residue that could serve as a site for palmitoylation (Hrabak et al., 2003; Harper et al., 2004; Hegemann et al., 2006). Furthermore the comparative syntenic analysis between Cucumis melo L. and Arabidopsis thaliana revealed that $13 \mathrm{CmCDPKs}$ and $19 \mathrm{AtCDPKs}$ existed in 20 corresponding syntenic blocks (Zhang et al., 2017). Genes like the CmCDPK and CmCRK were found differentially expressed in response to various stimuli like in biotic stress (Podosphaera xanthii) or abiotic stresses (salt and cold), and hormone (abscisic acid) treatment. This first 
comparative report on $C D P K$ and $C R K$ gene families in case of melon became a base for the functional characterizations of $\mathrm{CmCDPK}$ and $\mathrm{CmCRK}$ genes for future study approach (Zhang et al., 2017).

CDPKs are at the forefront of decoding transient $\mathrm{Ca}^{2+}$ signals into physiological responses in plants playing a very important role in many aspects of plant life from pollen tube growth, plant development, and in stress response to senescence and cell death (Simeunovic et al., 2016). Accordingly, most CDPKs have a distinct subcellular distribution which enables them to sense local $\mathrm{Ca}^{2+}$ concentration and to interact with its specific targets (Simeunovic et al., 2016). The CDPK family has been reported to be important not only in the higher plants but also in unicellular protists and green algae (Hamel et al., 2013; Baba et al., 2019).

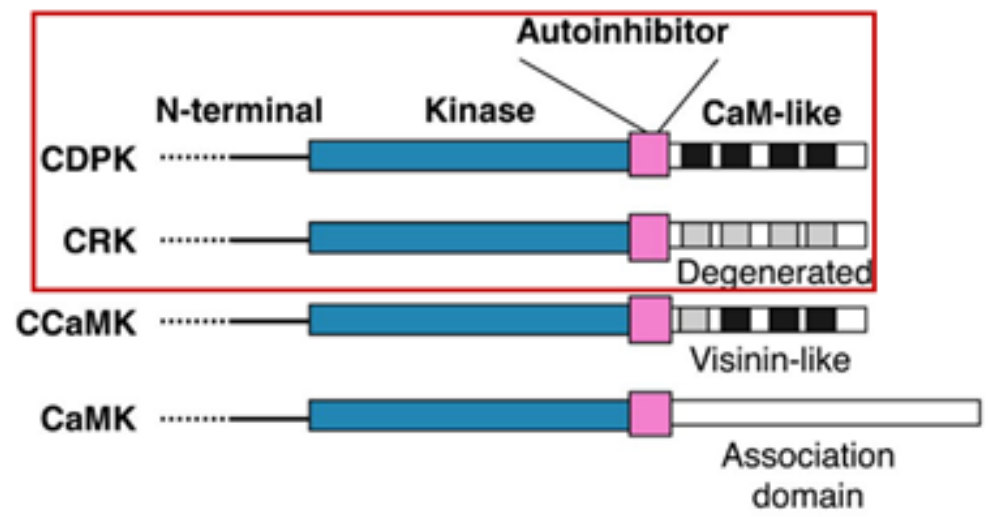

Figure 1: Domain structure of CDPKs, CRKs and two other related kinases. The N-terminal region is highly variable in both length and sequence of the domain. Autoinhibitor is predicted in the region immediately near the kinase domain. A distinguishing feature of CDPKs, CDPK-related protein kinases (CRKs) and calcium and calmodulin-dependent protein kinases (CCaMKs) is the number of functional EFhands in a C-terminal regulatory domain: EF-hands that can bind calcium are denoted by black boxes, whereas degenerated EF-hands are denoted by gray boxes (Harmon et al., 2000).

CDPK functions have been predominantly characterized in abiotic stress and immune signaling responses, with little focus upon their function in long-term adaptive processes or plant development has been reported. The identification of in vivo/in planta CDPK targets, including their respective phosphorylation sites, leads to elucidation of CDPKs function on a molecular and biochemical level. Moreover, the resolution of kinasespecific phosphorylation patterns within a target protein provides mechanistic evidence suggesting that CDPKs act as signaling hubs in plant stress signaling and development (Schulz et al., 2013). Another important factor of CDPKs is their subcellular localization, which ranges from their broad distribution throughout the cell. Confinement to a specific compartment of cell can add considerable specificity to them as it limits the pool of 
accessible target proteins and determines which calcium signals can be sensed. Finally, CDPKs also show different $\mathrm{Ca}^{2+}$ affinities, calcium dependency, as well as their regulation by (auto) phosphorylation (Boudsocq and Sheen, 2012). Together, with all these factors will determine where, when, and which kind of calcium signal will activate a specific CDPK for a potential response.

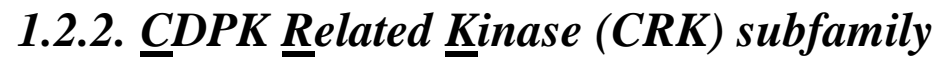

In Arabidopsis sp., CRK subfamily is structurally closely related to CDPKs and consists of eight members (Harper et al., 2004; Reddy et al., 2011). Unlike CDPKs and SnRKs, limited information is available about the functional role of plant CRKs in vivo in Arabidopsis plants. For a decade, only predictions of their membrane localization have been reported (Podell and Gribskov, 2004). All so far known CRKs, including AtCRK5, share a conserved C-terminal calmodulin $(\mathrm{CaM})$ binding domain, which overlaps with the kinase auto inhibitory domain (Zhang et al., 2002). The N-terminal region of most CRKs is predicted to be myristoylated and palmitoylated (Renna et al., 2013; Rigó et al., 2013). Biochemical studies with recombinant CRKs from carrot (Lindzen and Choi, 1995; Farmer and Choi, 1999) and maize (Furumoto et al., 1996) have shown that these representative enzymes do not bind, and are not activated by calcium. Rice CRK binds calmodulin in a calcium-dependent manner, although its enzymatic activity is independent of calcium and calmodulin (Zhang et al., 2002). In tomato (Lycopersicon esculentum) LeCRK1 was reported to encode a novel isoform of calcium-dependent protein kinase (CDPK) which was also isolated by screening a tomato cDNA library (Leclercq et al., 2005). The derived protein from this full-length sequence showed that it belongs to the family of CDPKrelated kinases (CRKs) and its predicted amino acid sequence display a modular organization of the protein consisting of different characteristic domains (Leclercq et al., 2005).

Previously, a detailed description of the whole CRK protein kinase family consisting of six members was reported in tomato, and they reported the first tomato CRK gene (SlCRK6) carrying disease resistance against plant pathogen (Wang et al., 2016). CRKs solely have a Ser/Thr kinase domain at their N-terminal region. Their C-terminal only contains degenerated EF-hand-like sequences. While, CDPKs function in a calciumdependent way, the CRKs are thought to act in a calcium-independent way (Harmon et al., 2000). No further study is available about majority of the AtCRKs function in vivo or its potential physiological substrates, except the few members like AtCRK5 protein kinase 
which was shown to be involved in the regulation of the root gravitropic response (Rigó et al., 2013). In the T-DNA insertion mutant of the AtCRK5 protein kinase the asymmetric auxin gradient was disturbed, causing the delayed gravitropic bending of Atcrk5 mutant and this consequence was reported due to improper phosphorylation of PIN2 protein (Rigó et al., 2013). Since the AtCRK5 kinase phosphorylates the hydrophilic loop region of the PIN2 molecule, the inactivation of the AtCRK5 protein kinase results in the lack of phosphorylation of the PIN2 protein in the Atcrk5 mutant resulting in improper polar localization of the PIN2 protein and as a result the auxin distribution is also altered in the root. However, no collapse in the root tip was observed in the seedlings and also the transcription of various genes for auxin biosynthesis, auxin influx, and PIN-FORMED (PIN) efflux carriers was found unaffected by the mutation in the AtCRK5 (Rigó et al., 2013).

Moreover the other member of AtCRKs like AtCRK1 (also mentioned as AtCBK3) was reported to bind calmodulin in a $\mathrm{Ca}^{2+}$-dependent manner, while autophosphorylation and substrate phosphorylation activity was found to be independent of $\mathrm{Ca}^{2+}$ (Wang et al., 2004). The Atcrkl mutant had impaired basal thermotolerance and the overexpression of AtCRK1 gene resulted in the increased basal thermotolerance in plants. AtCRK1 was shown to regulate transcription of heat shock protein (HSP) genes and synthesis of HSPs. The binding activity of HSF to the heat-shock element (HSE) and the mRNA level of HSP genes and synthesis of HSPs were upregulated in AtCRK1 overexpressing lines after heat shock, but was found to be downregulated in AtCRK1 null lines indicating that AtCRK1 controls the binding activity of HSFs to HSEs by phosphorylating the transcription factor AtHSFA1a, which is an important member of the HS signal transduction pathway (Liu et al., 2008). AtCRK1 was also shown to be involved in the tolerance to salt stress by changing both malondialdehyde (MDA) and proline levels in Arabidopsis, and might act as a positive regulator in the induction of stress-response gene transcription as the transcription of several stress-related genes was decreased in Atcrkl mutant plants. (Tao et al., 2013). Other member AtCRK3 was reported to interact with a cytosolic glutamine synthetase AtGLN1;1 and regulate nitrogen remobilization during leaf senescence (Li et al., 2006). Three Arabidopsis CRKs like; AtCRK2, AtCRK3, and AtCRK8 were shown to phosphorylate the C-terminal tyrosine (Tyr) residues of $\beta$-tubulin (TBB-2 and TBB-7), which are the major structure of microtubules. Moreover, AtCRK2 and AtCRK3 had high in vitro Tyr-phosphorylation activity for some transcription factors like ethylene response factor 13 (ERF13), WRKY DNA-binding protein 14 (WRKY14), ERF subfamily B-4 of 
ERF/AP2 transcription factor 2.6(RAP2.6), and cryptochrome-interacting basic helix-loophelix5 (CIB5) (Nemoto et al., 2015). AtCRKs, in addition to their Ser/Thr phosphorylation capability, were reported to posses Tyr kinase activity, assuming their roles in Tyr phosphorylation signaling in angiosperms (Nemoto et al., 2015).

\subsection{Auxin transport and its signaling}

\subsubsection{Auxin an important hormone in plant development}

Auxin an important plant hormone (the predominant form of which is indole-3acetic acid; IAA) is a major coordinating signaling molecule in the regulation of various forms of plant development (Petrásek and Friml, 2009). Auxin is one of the most investigated plant hormones known and is involved virtually in every form of plant growth and development such as, embryogenesis, organogenesis, tissue patterning and tropisms (Quint and Gray 2006; Davies 2010; Saini et al., 2013). It is a weak organic acid consisting of a planar indole ring structure coupled to a side chain harbouring a terminal carboxyl group. The carboxyl group is protonated at low $\mathrm{pH}$, making the molecule less polar and in this state it can diffuse across the cell membranes (Figure 2); (Ljung, 2013).

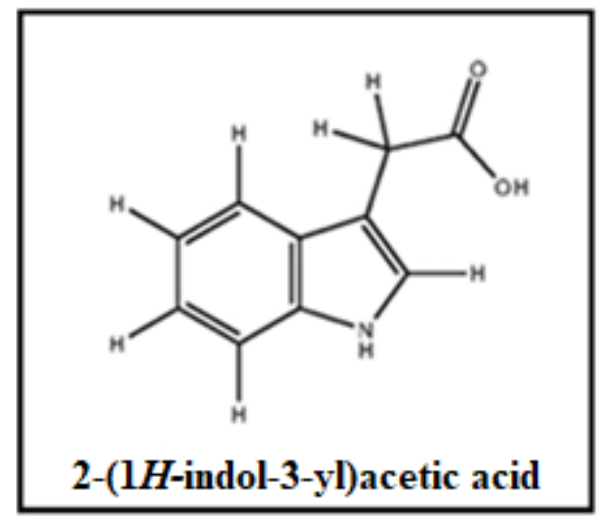

Figure 2: Chemical structure of auxin (Indole-3-acetic acid). The chemical structure and IUPAC name for IAA (Ljung, 2013).

Though the most common naturally occurring auxin is IAA, but other forms of auxin include indole-3-butyric acid (IBA) and 4- chloroindole-3-acetic acid (4-Cl-IAA). Functionally analogous, synthetic auxins such as 1-naphthylacetic acid (1-NAA) and 2,4dichlorophenoxyacetic acid (2,4-D) are metabolically more stable and, because of the stability and analogy with naturally occurring auxin, they are more widely used in agricultural applications and biological studies (Taiz and Zeiger, 2010). Interestingly, the exogenous application of each auxin species leads to a distinct but partially overlapping scale of desired effects (Normanly et al., 2004; Tanaka et al., 2006). This partially 
overlapping spectrum of effects is possibly due to the specific transport properties for each type of auxin species, and their differences in tissue permeability and stability. IAA is structurally related to and derived from tryptophan (Trp). However, Trp is not absolutely necessary for its synthesis, and several pathways that are both dependent and independent of Trp, have been proposed for auxin biosynthesis. In addition, IAA can be inactivated or stored by conjugation to amino acids, sugars and peptides (Normanly et al., 2004; Woodward and Bartel, 2005; Tanaka et al., 2006).

\subsubsection{Mechanism of directional auxin transport}

To explain the mechanism of directional cellular polar auxin transport from cell to cell, the best postulated model of chemiosmotic hypothesis was based on a $\mathrm{pH}$ gradient across the plasma membrane which leads to the accumulation of IAA in the cell. In the cell wall there is the acidic environment $(\mathrm{pH} \mathrm{5.5),} \mathrm{and} \mathrm{the} \mathrm{IAA} \mathrm{molecule} \mathrm{is} \mathrm{present} \mathrm{in} \mathrm{both}$ ionized and protonated forms. This protonated IAAH being hydrophobic in nature enters the plasma membrane through passive transport. Once inside the more basic cytoplasm $(\mathrm{pH} 7.0)$, the proton dissociates and the IAA ion cannot passively move out of the cell and therefore becomes trapped inside (Figure 3). Specific auxin efflux transporters are needed to transport these IAA ions out of the cell (Morris et al., 2010). Besides the passive diffusion of auxin, this molecule is also actively taken up from the apoplast by $\mathrm{H}^{+} / \mathrm{IAA}^{-}$symport mediated by AUXIN-RESISTANT 1/LIKE AUX1 (AUX1/LAX) influx carriers (Vanneste and Friml, 2009). Once inside the neutral cytosol, auxin is deprotonated and becomes trapped inside the cell and can leave the cell by auxin efflux carriers such as PIN-FORMED (PIN) proteins and P-glycoproteins (PGP) of the ATP-binding cassette family B (ABCB) transporter family (Vanneste and Friml, 2009). The polar subcellular localization of PINs determines the direction of auxin flow out of the cell and thus the unidirectional auxin flow within tissues (Vanneste and Friml, 2009). 


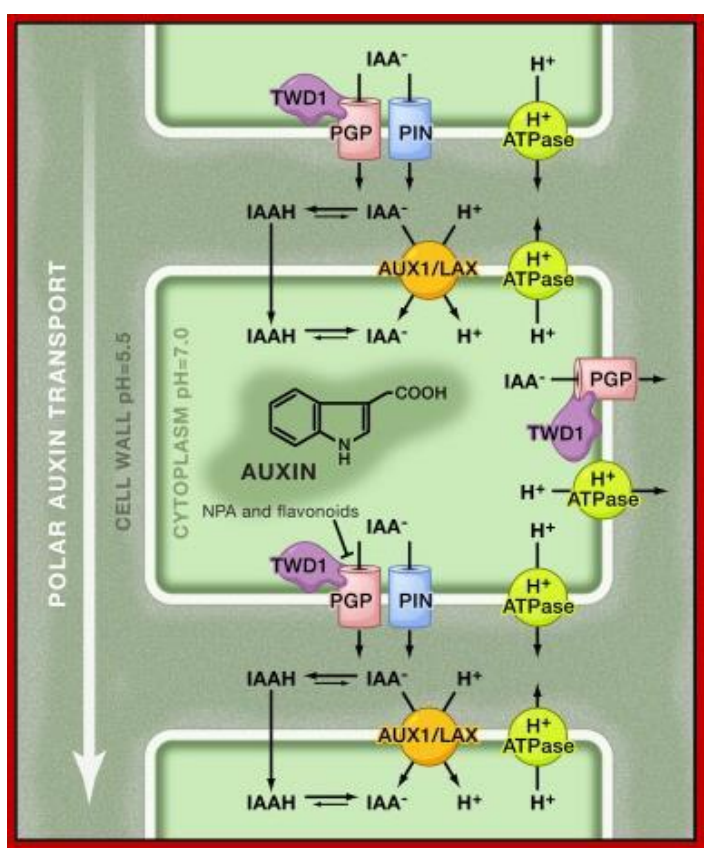

Figure 3: Chemiosmotic hypothesis for polar auxin transport. The low $\mathrm{pH}$ in the apoplast (cell wall) is maintained through the activity of plasma membrane $\mathrm{H}^{+}$ATPases. In the relatively acidic environment, a fraction of the weak acid, indole-3-acetic acid (IAA), the major form of auxin, becomes protonated. The protonated (IAAH) form is more lipophilic and can diffuse freely through the plasma membrane into the cell. (Vanneste and Friml., 2009).

The dynamic, differential distribution of the hormone auxin within plant tissues controls a variety of developmental processes, which progresses plant growth and morphological changes to various environmental conditions. Various environmental and endogenous signals can be integrated into changes in auxin distribution through their effects on local auxin biosynthesis and intercellular auxin transport. Many functions of auxin action depend on its differential distribution within plant tissues, forming the local maxima between cells. The major determinant of differential auxin distribution is its directional transport between cells and formation of polar auxin distribution. This regulated polar auxin distribution and transport (PAT) through various influx and efflux transporters within plant tissues appears to be unique to this hormone (Petrásek and Friml, 2009). Besides auxin it was also reported the role of gibberellic acid in higher levels at the lower side of the root correlate with increased amounts of PIN-FORMED2 (PIN2) auxin transporter at the plasma membrane, displaying the role of other plant hormone during asymmetric action during gravitropic responses (Löfke et al., 2013).

\subsubsection{Auxin transporters}

In plant kingdom many recent studies of several asymmetrically localized plasma membrane (PM) transport proteins have been documented. They play a crucial role in mechanism of cell polarity and the plant development. These proteins include one of the 
important auxin efflux transporters called as PIN-FORMED and the most studied family of auxin efflux carriers controlling auxin transport is the PIN proteins. These proteins are polar-localized transmembrane auxin efflux carriers that play an important role in the polar auxin transport and are implicated in diverse plant developmental processes. They display a distinct apical, basal or lateral polarity in the PM, depending on the type of PIN transporter and the tissue of the plant (Ganguly et al., 2012). Their name was derived from the characteristic of pine (tree) needle (pine leaves) like shape flower axis of the pin1 (pinform-1) mutant phenotype (Goto et al., 1987). Various other auxin transporters reported to facilitate the polar auxin transport (PAT), such as already mentioned AUX1, LAX1, LAX2 and LAX3 (Peret et al., 2012; Swarup et al., 2012), also the ATP-binding cassette $(\mathrm{ABC})$ transporters, nitrate transporter 1.1 (NRT1.1), PIN-like transporters (PILS) and WALLS ARE THIN 1 (WAT1) (Zhou and Luo, 2018). Members of the (MDR)-type ATP-binding cassette (ABC) protein family like the AtMDR1 and Arabidopsis thaliana P-glycoprotein1 (AtPGP1), identified earlier were being functionally related to anion channels; regardless their corresponding mutants and double mutants displayed auxin-related phenotypes including a decreased rate of auxin transport (Noh et al., 2001; Friml, 2003).

The ABCB proteins together with the PINs stimulate the directional auxin transport (Taiz and Zeiger, 2010). Interestingly, ABCB19 is localized at the plasma membrane of epidermis at the inner side of the apical hook, likely mediating basipetal auxin flow through this side (Noh et al., 2001; Wu et al., 2010). The polarity of the plasma membrane localization of PIN auxin efflux transporters determines the directionality of intercellular (polar) auxin transport and have been shown to impose differential auxin gradient within specific tissues (Wabnik et al., 2011). The delivery of these important auxin carriers, such as PIN proteins to their specific target sites requires defined vesicle trafficking routes. Cellular mechanisms that guide vesicle transport determine the subcellular localization, activity and abundance of auxin carriers in the region (Löfke et al., 2013). Previously, in silico screening for novel PIN-like putative auxin transport facilitator family revealed a topologically similar protein transporters like PIN protein family consisting of seven members in Arabidopsis thaliana designated as the PIN-LIKES (PILS) proteins (Barbez et al., 2012). PILS were potentially found to play role in auxin-dependent regulation of plant growth by determining the cellular sensitivity to auxin, regulating intracellular auxin accumulation at the endoplasmic reticulum and thus auxin availability for nuclear auxin signaling (Barbez et al., 2012). 


\subsubsection{Auxin efflux PIN transporters}

The PIN gene family encodes a subgroup of auxin efflux carriers, which costs energy from the electrochemical gradient across the PM (Zažímalová et al., 2007; 2010). The distinct feature of this subfamily of PINs is their central hydrophilic loop separating two hydrophobic domains of about five transmembrane regions (Figure 4). On the basis of the size of the central hydrophilic loop, the PIN proteins can be generally divided into two major subgroups, the "long" and "short" PIN proteins (Krecek et al., 2009). PIN1 was the first identified PIN gene in Arabidopsis, afterwards seven additional PIN genes were reported in the genome of Arabidopsis (Křeček et al., 2009; Adamowski and Friml, 2015; Zhou and Luo, 2018). To date, PIN genes have been identified in by genome-wide approach in a number of plant species and these identified PIN genes range from 4-23, reporting the lowest and highest numbers of PIN genes were found in Glycine max and Marchantia polymorpha, respectively (Wang et al., 2015; Liu and Wei, 2017; Zhou and Luo, 2018). As polarity is one of the most important features of plants at cellular level and can be seen in various tissues and organs so in case of PIN protein efflux transporters their polarity is directional with the auxin flow in cells and tissues in plants. Plants can redefine their cellular and tissue polarity based on the influence of various factors. Auxin has been identified as an important factor for tissue and organ polarity in plants, mainly on account of its controlled directional (polar) flow through plant tissues (Zhou and Luo, 2018).

Eight PIN proteins have been reported in Arabidopsis plant. PIN classification in Arabidopsis included, PIN1-PIN4, PIN6 and PIN7 as long PIN proteins and the PIN5 and PIN8 are considered as short PIN proteins (Křeček et al., 2009; Viaene et al., 2013; Zhou and Luo, 2018). The members like PIN1, PIN2, PIN3, PIN4 and PIN7 proteins are localized towards plasma membrane (Feraru and Friml, 2008), while the others like PIN5, PIN6 and PIN8 proteins are endoplasmic reticulum (ER) localized (Ganguly et al, 2012; Zhou and Luo, 2018). Some long PIN proteins with reduced long hydrophilic loop (HL), such as PIN6 in Arabidopsis, posseses dual location at both the PM and ER depending on its state of phosphorylation (Simon et al., 2016). The localization of these PIN transporters is polar, and corresponds with the direction of the auxin flow. The distinct feature of this subfamily of PINs is their central hydrophilic loop separating two hydrophobic domains of about five transmembrane regions (Figure 4). On the basis of the size of the central 
hydrophilic loop, the PIN proteins can be generally divided into two major subgroups, the "long" and "short" PIN proteins (Krecek et al., 2009).

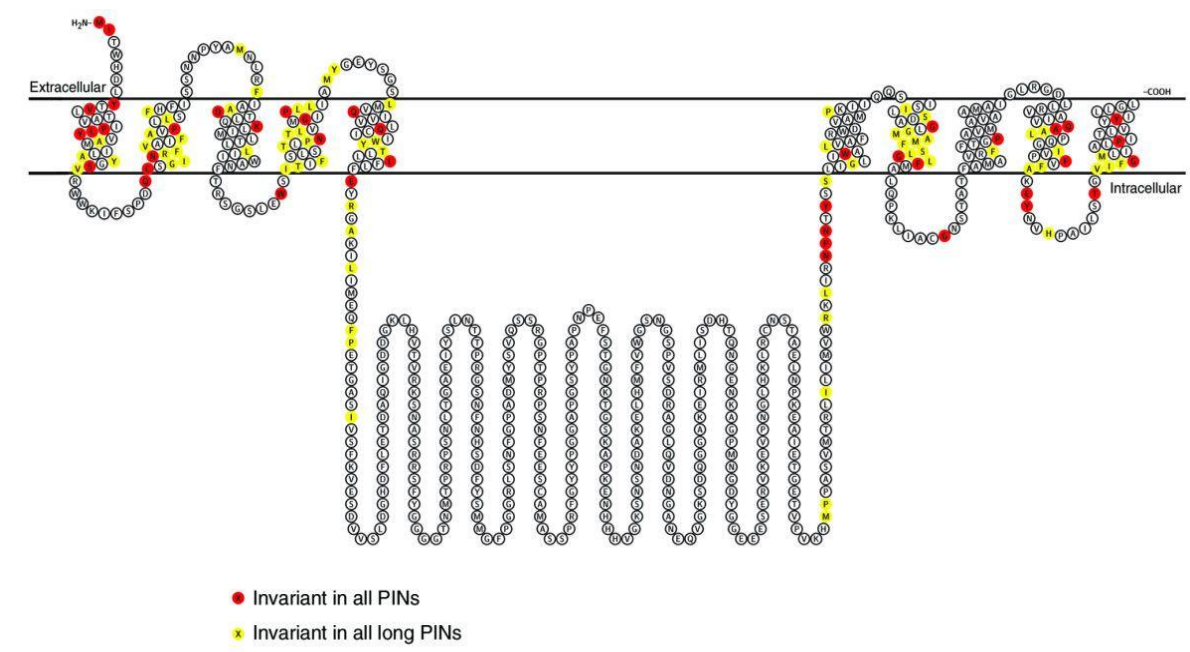

Figure 4: The predicted structure of PIN proteins. The given sequence shown from AtPIN7; the positions marked in yellow are invariant in sequences of all 'long' PINs, the positions marked in red are invariant in sequences of all PINs (Křeček et al., 2009).

The subfamily of long PINs encompasses all members of the family that are defined as important auxin-efflux carriers like PM localized PIN1-PIN4 and PIN7 and their homologs from seed plants - called the canonical PINs) (Křeček et al., 2009). The localization and activities of these PIN proteins are affected by their phosphorylation status (Křeček et al., 2009; Offringa and Huang, 2013; Luschnig and Vert, 2014; Barbosa et al., 2018).

\subsubsection{Auxin influx protein transporters}

Besides the various auxin efflux transporters there are also other specific auxin influx transporters, such as the AUX1/LAX family, which are also important in transporting IAA into specific cell types (Swarup and Péret, 2012). AUX1 belongs to the auxin amino acid permease (AAAP) family of proton-driven transporters (Bennett et al., 1996). AUX1 was reported to display dual auxin transport function in the root apex of Arabidopsis, in promoting the acropetal and basipetal auxin transport in protophloem and lateral root cap cells, respectively (Swarup et al., 2001). In the Arabidopsis development during embryonic root apical meristem (RAM) requirement of active transport of the signaling molecule auxin, leads to its relocation from its origin to establish a morphogenetic field causing changes in the gene expression in individual cells (Bhalerao and Bennett, 2003; Friml, 2003; Benková et al., 2009; Ugartechea-Chirino et al., 2010; 
Robert et al., 2015). It was previously demonstrated that members of the AUX1/LAX family of proteins are redundantly required for correct cellular organisation in the case of the tip of radical in mature embryos (Ugartechea-Chirino et al., 2009). Later the AUX1/LAX-dependent auxin influx carriers were evidenced to define cellular patterning from early embryogenesis onward and also the expression pattern of $A U X 1$ and LAX2 is controlled by the MONOPTEROS-BODENLOS (MP-BDL) signaling pathway (Robert et al., 2015). A cooperative role of the auxin influx (LAX1) and efflux (PIN1) transporters in cotyledon specification is also reported to be important and support the requirement of coordinated auxin influx and efflux for proper embryo development (Robert et al., 2015).

\subsubsection{Polar localization and trafficking of PINs}

This polar auxin transport in the plants together with its physiological and developmental roles in response to environmental stimuli has been hypothesized that the combinations of environmental and endogenous factors regulate the auxin flow in different plant tissues. This revealed information about the directional flow of auxin in controlling growth (Went, 1974; Feraru and Friml, 2008). The asymmetric pattern of auxin distribution regulates a variety of different processes during plant growth and development. The polar auxin transport that generates auxin patterns is determined by polar localization of PIN proteins at a specific side of the plasma membrane (PM). These PIN transporters in roots possess a defined localization pattern like the PIN1 is localized at the basal (root apex-facing) side of the root vasculature; PIN2 at the basal side of the cortical cells and at the apical (shoot apex-facing) side of the epidermal and root cap cells; PIN3 localizes in an apolar manner in the columella cells of the root; PIN4 at the basal side of cells in the central root meristem and in the cells of the quiescent center they are less pronounced; and PIN7 at the basal side of the stele cells and apolar in the columella cells. The polar localization of some specific PIN transporters in root is depicted below in the (Figure 5); (Feraru and Friml., 2008). 

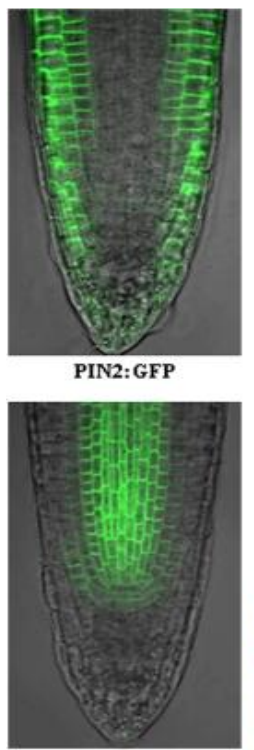

PIN1:GFP

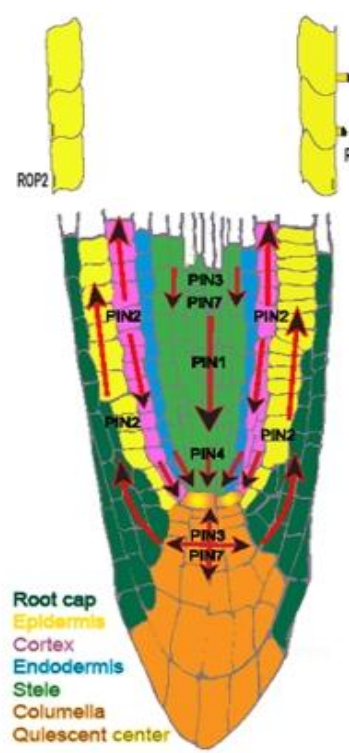

Columella

Quiescent cen:

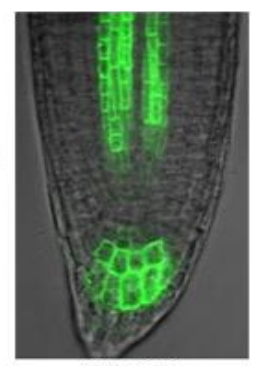

PIN7: GFP

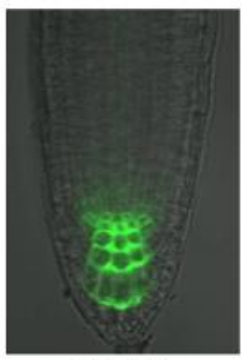

PIN4: GFP

Figure 5: Polar localization of PIN proteins in the Arabidopsis root tip. Direction of auxin transport (arrows) is determined by the polar, subcellular localization of PIN proteins. PIN1:GFP, PIN2:GFP, PIN4:GFP and PIN7:GFP expressions in the root are also depicted (Feraru and Friml, 2008).

In Arabidopsis in the case of short PINs like PIN5, it is reported to express relatively weak and ubiquitously in the aerial part of the plant with highest expression in the hypocotyl, the guard cells of stomata and cauline leaves (Mravec et al., 2009). The other transporter PIN8 is known to have a specific expression pattern exclusively in the male gametophyte - the pollen. These short PINs (typically PIN5 and PIN8) have been reported to be localized mainly to the endoplasmic reticulum, where they function to distribute auxin flow between the cytoplasm and endoplasmic reticulum lumen and regulate subcellular auxin homeostasis (Křeček et al., 2009). In case of the PIN6 the central hydrophilic loop is significantly known to be reduced and the PIN6 is predominantly known to be localized in the endoplasmic reticulum membrane (Mravec et al., 2009). Recently the genetic, molecular and pharmacological approaches were applied to characterize the molecular mechanisms that control the subcellular localization of PIN6 in Arabidopsis. It was reported that its localization is under the control of developmental signals acting on tissue-specific determinants controlling PIN6 protein levels and PIN6 phosphorylation (Ditengou et al., 2018).

It has been shown that PIN proteins cycle between the PM and intracellular compartments through different protein trafficking pathways (Geldner et al., 2001; Offringa and Huang, 2013; Luschnig and Vert, 2014; Pan et al., 2015). These PIN proteins are under continuous process of endocytosis and exocytosis, which act in both coordination and maintenance of PIN polarity (Kleine and Friml, 2008). This transport of PINs was 
blocked on the treatment of a fungal toxin brefeldin A (BFA) which inhibits the trafficking or exocytosis of PIN proteins from the endosomes to the PM, resulting in PIN accumulation into so-called BFA compartments inside the cell (Geldner et al., 2001). Despite the changes in PIN polarity in response to various internal signals, PIN polarity switching can also occur in response to a series of external environmental stimuli like in case of response to gravity. After gravistimulation, it was reported that PIN3 could relocate to the basal side of columella cells and thus rerouting the auxin flow to the lower side of the root and triggering root bending response (Friml et al., 2002; Harrison and Masson, 2008, Rigo et al., 2013). This defined polarity of PINs in response to various external signals defines how the signal integration at the level of subcellular dynamics of individual cells can be translated into the directional signaling at the tissue level and regulates to the plasticity of plant development (Feraru and Friml, 2008).

\subsubsection{Embryogenesis in Arabidopsis and the role of auxin transporters}

\subsubsection{Embryo development and its specification}

Embryogenesis initiates from a single cell, the zygote, and then end with a mature embryo with specific and organized fate attained by the cells. This organized division of cells and their specification during embryogenesis is described as the pattern formation. The defined processes that initiate all of these different cell types from the zygote remain largely unknown. As reported the plant hormone auxin plays a crucial role in the development of the embryo and its specification (Möller and Weijers, 2009). Thus the important pattern formation in Arabidopsis depends on the auxin biosynthesis, transport, and response in the embryo. This defined embryo patterning steps involve auxin activity and the molecular mechanisms of auxin action during this phase of plant development (Möller and Weijers, 2009).

Auxin concentrations during cellular development can be ascertained by the induction involving the gene expression of auxin sensing reporter DR5 fused with green fluorescent protein GFP (Ottenschläger et al., 2003). DR5 reporter activity can depict the sites of auxin activity during embryogenesis (Friml et al., 2003; Möller and Weijers, 2009). Thus, regulated auxin concentrations and activity are needed for the organization of both the apical and basal embryo development and are therefore critical for the establishment of the embryo pattern (Möller and Weijers, 2009). Recently it was evidenced the role of the maternal tissue in contributing to the source of auxin supply for early embryogenesis, 
coordinating the seed development by the auxin to the early embryo from the integuments of the ovule (Robert et al., 2018). This auxin response in the ovules increases on fertilization because of the upregulation of auxin biosynthesis in the integument leading to correct embryo development (Robert et al., 2018).

\subsubsection{Expression and polarity of auxin infux/efflux transporters in embryos}

As the PIN proteins have cellular and tissue specificity like in the process of embryogenesis, PIN1, PIN4, and PIN7 show polar localization and act together to specify the apical-basal axis of the embryo (Adamowski and Friml., 2015). In postembryonic stages and development, PIN proteins have different PM localizations, most being localized at the basal (root apex-facing) side of the vasculature and stele cells, such as PIN1, PIN3, PIN4, and PIN7, whereas some localize also apically (shoot apex-facing side), such as PIN1 in the shoot apex epidermis or PIN2 in the lateral root cap and epidermis cells (Feraru and Friml, 2008). During proembryo development, PIN7 localizes to the apical sides of suspensor cells leading to auxin flow into the proembryo, where PIN1 is localized first in a nonpolar manner. During the middle globular embryo stage, PIN1 polarity in provascular cells is formed at basal part, followed by PIN7 relocation to the same side of suspensor cells. Recently it was observed that the initial expression of PIN3 protein was observed within the ovule integuments (Robert et al., 2018) and also has been earlier reported towards the basal pole of the heart stage embryo (Friml et al., 2003). This polarity of PIN rearrangements results in redirection of the auxin flow from the apical part to the basal part of the embryo, where auxin is accumulated and then plays role in specification of root meristem (Friml et al., 2003). Further the PIN4 expression in the central root meristem displays its role during later stages in the establishment of local auxin accumulation in the center of developing root meristems. PIN1 further relocates at the apical surface of the proembryo leading to establishment of two symmetrically positioned auxin accumulation foci marking sites of future embryonic leaves (Figure 6); (Friml et al., 2003; Feraru and Friml, 2008). 

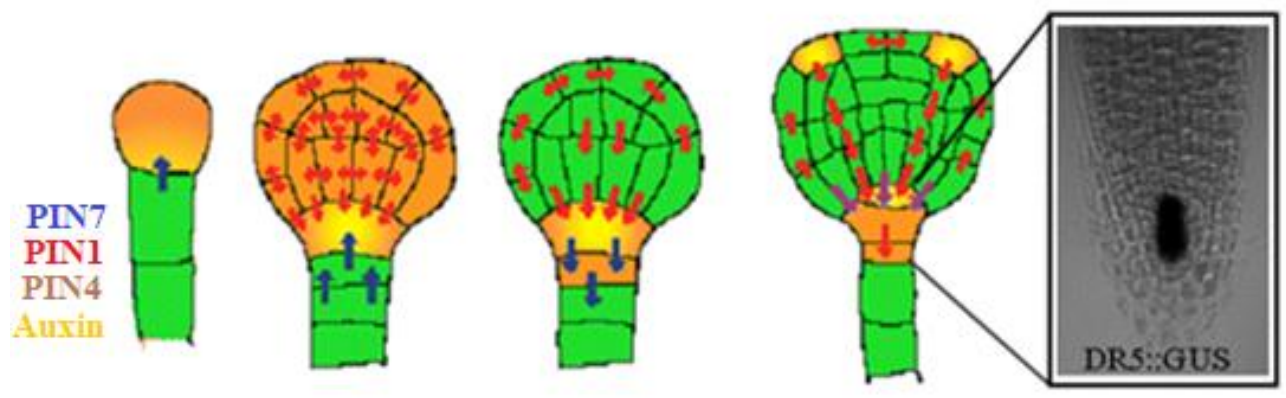

Figure 6: Formation of apical-basal polarity during Arabidopsis embryogenesis. Polar localization of PIN1, PIN4 and PIN7 transporters and the formation of an auxin gradient at various embryo stages. Light microscopic image represents the root DR5::GUS ( $\beta$-glucuronidase) signal (Feraru and Friml, 2008).

AUX/LAX family of auxin influx facilitators participates in the establishment of patterning in cells within the apex of the embryonic root in a gene-redundant fashion. It was demonstrated that auxl and lax mutants are affected in case of their functionality in cell proliferation and cell growth within the radicle tip. So the importance of AUX/LAX auxin importers emerge as novel players in morphogenesis involved in patterning during embryonic root formation (Ugartechea-Chirino et al., 2010; Robert et al., 2015). Previously identified a cooperative role of auxin influx (LAX1) and efflux (PIN1) in cotyledon specification was shown supporting the requirement of coordinated auxin influx and efflux for proper embryo development (Robert et al., 2015). The expression of the auxin influx carrier AUX1 is initially detected in the central cells of a 32-cell stage embryo with LAX2 expression from the 32-cell stage onwards (Robert et al., 2015).

\subsubsection{Auxin signaling}

Auxin can mediate a variety of developmental processes by acting as a general signal for the change in developmental program in cells where it is distributed and thus providing directional information to the tissues by its polar intercellular flow (Vieten et al., 2007). Currently there are two most commonly reported proteins to function as auxin receptors; the first one described as AUXIN BINDING PROTEIN 1 (ABP1) and the other auxin receptor protein accepted as TRANSPORT-INHIBITOR-RESISTANT1 (TIR1); (Scherer, 2011). The later known to for a complex called the nuclear $\mathrm{SCF}^{\mathrm{TIR} 1 / \mathrm{AFBs}}{ }_{-}$ Aux/IAA (SKP-Cullin-F box [SCF], TIR1/AFB [AUXIN SIGNALING F-BOX], AUXIN/INDOLE ACETIC ACID) auxin coreceptors (Dharmasiri et al., 2005; Kepinski and Leyser, 2005; Salehin et al., 2015). 
Both of these auxin receptors are described to bind specifically and reversibly to auxin. ABP1 binds two molecules of auxin as a dimer and TIR1 binds auxin in a complex consisting of TIR1, auxin and an IAA protein (Scherer, 2011). The best reported candidate for an auxin receptor mediating auxin-regulated cell expansion is ABP1 (Batt et al., 1976; Ray et al., 1977) and the thorough characterization of this receptor was carried out extensively since its discovery. ABP1 is localized in the ER lumen with 1-3\% secreted to the extracytosolic side of the plasma membrane where it binds auxin (Effendi et al., 2013), arbitrating the fast auxin responses involving the cell wall loosening, cytoskeleton rearrangement and regulation of endocytosis, leading to cell expansion (Figure 7); (Ljung, 2013). ABP1 has been shown to bind auxins at concentrations corresponding to physiological concentrations for their activities (Jones and Venis, 1989; Chen et al., 2001; Scherer, 2011).

Various initial genetic screens revealed several candidates of the protein ubiquitination machinery involving the particular components and regulators of a SCF E3 ubiquitin ligase, such as the F box component TIR1 (Leyser et al., 1993; Vanneste and Friml, 2009). Environmental and internal signals can be accompanied by changes in auxin distribution through their effects on localized auxin biosynthesis and intercellular auxin transport. Cells interpret auxin largely by a nuclear defined signaling pathway that involves the $\mathrm{F}$ box protein TIR1 acting as an auxin receptor. As a result of auxin accumulation, auxin signaling, dependent on complex formation of cellular $\mathrm{SCF}^{\mathrm{TIR} 1 / \mathrm{AFB}}$, Aux/IAA, and auxin response factors (ARFs), triggering specific changes in developmental programs by activating expression of various defined genes, those coding for components of auxin transport and auxin synthesis (Ljung, 2013).

In addition to this defined mechanism of auxin transport, auxin signaling is also in controlled feedback mechanism that regulates the rate and polarity of its own transport. So when the auxin concentrations are low, Aux/IAAs are more stable and can interact with ARFs to inactivate them by the participation of TOPLESS (TPL) transcriptional corepressor and when the levels of auxin concentration increase, it causes the stabilization of the interaction between TIR1/AFBs and Aux/IAA proteins. With SCF TIR1/AFBs participation, the Aux/IAAs become ubiquitinated and targeted for proteolysis, resulting in modulation of transcription finally (Figure 7); (Vanneste and Friml, 2009; Ljung, 2013). 


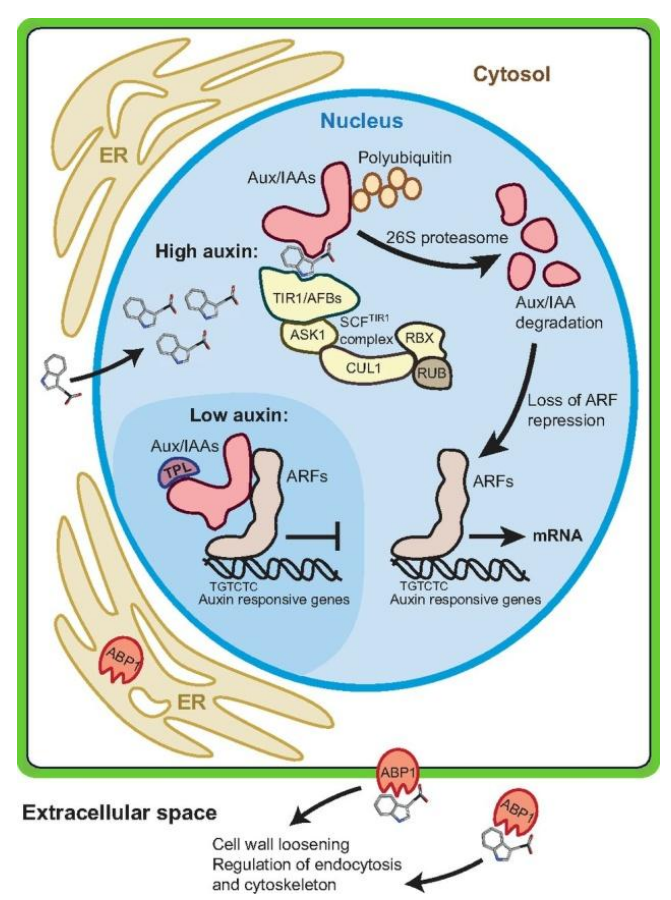

Figure 7: Components in auxin perception and signaling (Ljung, 2013).

\subsubsection{Hormonal crosstalk during embryogenesis}

Bioactive gibberellins $\mathrm{GA}_{1}, \mathrm{GA}_{3}$ and $\mathrm{GA}_{7}(\mathrm{GAs})$ are diterpene hormones in plants that are biosynthesized through complex pathways and control diverse aspects of growth and development. Biochemical, genetical, and genomical study approach led to the identification of various genes that encode GA biosynthesis and deactivation enzymes (Yamaguchi, 2008). The clear understanding of GA biosynthesis and deactivation pathways that are tightly regulated by developmental, hormonal, and environmental signals, consistent with the role of GAs as key growth regulators (Yamaguchi, 2008). Bioactive GAs has also been observed to play a regulatory role during tropic responses by modifying of the plasma membrane prevalance of PIN auxin transporters by an unclear mechanism (Löfke et al., 2013; Salanenka et al., 2018). Both auxin and gibberellic acid (GA) are prominent growth regulator and their interactive signaling pathways show positive interaction to regulate cellular expansion and tissue differentiation (Weiss and Ori, 2007; Abbas et al., 2013; Salanenka et al., 2018). During embryogenesis various transcription factors like the LEAFY COTYLEDON LEC1, LEAFY COTYLEDON2 (LEC2) and FUSCA3 (FUS3) play a role in case of late embryogenesis in Arabidopsis (West et al., 1994; Luerssen et al., 1998; Stone et al., 2001; Braybrook and Harada 2008; Hu et al., 2018). The essential role of GA in late embryogenesis was recently detailed ( $\mathrm{Hu}$ et al., 2018). The hormonal interaction between auxin with abscissic acid (ABA) signaling was 
shown to control seed dormancy in Arabidopsis (Liu et al., 2013; Locascio et al., 2014). GAs and ABA are known to work antagonistically on seed developmental process (Ye and Zhang, 2012; Liu et al., 2013; Locascio et al., 2014; Shu et al., 2018). During seed maturation and desiccation the levels of ABA increases, while the content of active GAs decreases (Locascio et al., 2014). In contrast to ABA, the GA plays a role in germination by mobilizing the resources of embryo (Koornneef et al., 1982). During the bent cotyledon embryo stage some GA biosynthesis genes are reported to be activated followed by the various proteolytic enzymes and alfa - amylases (Sreenivasulu and Wobus, 2013). The increased level of auxin concentrations during seed development is also attributed and known to contribute the promotion of the biosynthesis of active GAs through its regulation involving the expression of many GA metabolism genes like gibberellin 20-oxidase (AtGA20ox) or gibberellin 2-oxidase (AtGA2ox) (Frigerio et al., 2006; Dorcey et al., 2009).

The hormonal cross network between auxin/gibberellin and the auxin transport (Schwechheimer, 2012; Abbas et al., 2013; Salanenka et al., 2018) displays the reduction in auxin transport in the inflorescence of Arabidopsis gibberellin biosynthesis and signaling mutant gal. Insufficeincy in the auxin transport was coherent with the reduced levels of PIN2 transporter abundance in this GA biosynthesis mutant (Willege et al., 2011). The external application of GA led to the restoration of the PIN2 protein levels to that of wild type level. As a consequence of the decreased GA levels it was thus reported that the PIN2 proteins were targeted for vacuolar degradation which leads to reduced auxin transport (Willege et al., 2011). Two key enzymes, GA20ox and gibberellin 3-oxidase (GA3ox), play a critical role in catalysis of the gibberellin intermediates into the bioactive forms, and the bioactive gibberellins are further catabolized by gibberellin 2-oxidase (GA2ox). The bioactive forms of gibberellins $\left(\mathrm{GA}_{1}, \mathrm{GA}_{3}\right.$ and $\left.\mathrm{GA}_{7}\right)$ are then recognized by soluble receptor GID1 and then activate the degradation of DELLA proteins that act as important repressors of gibberellin signaling (Hu et al., 2018). These bioactive gibberellins play an important regulatory role in the late embryogenesis involving the modulator DELLA proteins. It was thus reported that insufficient gibberellic acid levels and the DELLA protein accumulation displayed a phenotype leading to abnormal embryos with the shortening of embryo axis and affecting the bending of cotyledons ( $\mathrm{Hu}$ et al., 2018).

DELLA proteins involve a family of nuclear-localized proteins belonging to a subset of GRAS family of putative transcriptional regulators containing the specific DELLA motifs at their N-terminal and conserved GRAS domains at their C-terminal. They are key 
repressors of the GA signaling pathway (Fleet and Sun, 2005; Achard and Genschik, 2009; $\mathrm{Hu}$ et al., 2018). In Arabidopsis genome, there are five DELLA proteins, designated as GA INSENSITIVE (GAI), REPRESSOR OF gal-3(RGA), REPRESSOR OF gal-3-LIKE protein (RGL)1, RGL2, and RGL3, respectively (Peng et al., 1997; Lee et al., 2002). During the GA signaling, these DELLAs have been reported to execute either the canonical transcriptional regulation involving the physical interaction with transcription factors and other regulatory proteins preventing their binding to target genes or as coactivators in promotion of specific target gene expression (Daviere and Achard, 2016; Hu et al., 2018) or participating in a nontranscriptional way of GA signaling by redirecting the protein trafficking from the path involving vacuolar degradation to the plasmamembrane (Salanenka et al., 2018). In continuation with the late embryo development these DELLAs proteins interact with LEAFY COTYLEDON1 (LEC1) protein (Hu et al., 2018). During this specified tenure of embryo formation, the bioactive gibberellins are biosynthesized in increased quantity then in process triggering the degradation of DELLAs to achieve the transcriptional activity of LEC1, finally promoting the process of late embryo development (Locascio et al., 2014; Hu et al., 2018).

An increased level of auxin (AUX) present during the development of seeds suggests that this hormone plays key role throughout the entire program of seed formation (Locascio et al., 2014). Cytokinins (CK) accumulation is the opposite when compared with auxin levels. The brassinosteroids (BR) display similar patter like of CKs (Locascio et al., 2014). Accumulation of gibberellins (GA) is characteristic, showing two peaks which correspond to specific phases of seed development: first one involving the embryo differentiation, when the GAs promote cell growth and expansion, and the second one at the end of the maturation phase, when proteolytic enzymes are activated for carrying resources from the endosperm necessary for germination (Figure 8); (Locascio et al., 2014). The pattern of abscissic acid (ABA) shows an accumulation pattern antagonistic to the GAs, the main hormone that inhibits all the processes induced by GAs (Locascio et al., 2014). 


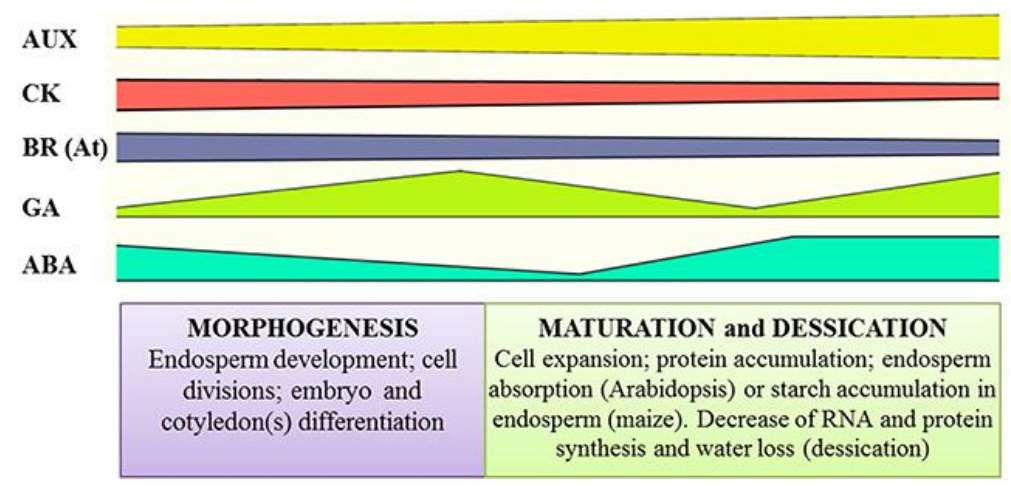

Figure 8: Schematic trend of hormone accumulation during seed development (Locascio et al., 2014).

\subsection{Root gravitropism}

Plants have an important feature of their ability to adapt to growth towards or away from external stimuli such as light, water, temperature or gravity. These movements of plant in response to specific stimuli are called tropisms and they play an important role in survival, reproduction and development of plant. The first detailed studies of tropisms were performed by Charles Darwin in the year 1880, where he described that etiolated grass coleoptiles shows the growth towards the source of light when it was illuminated from one side of the plant (Darwin, 1880). Plant gravitropism is one of the tropisms which govern the plant roots to bend towards the direction of gravity vector, thus serving as an important process for gravity response in plants. In roots the perception of gravity takes place in the columella cells in the root cap, but the gravitropic response takes place in the cells of elongation zone of root, and there is a distinct physical separation between the site of gravity perception and the site of gravitropic response (Sato et al., 2005).

Perception of a change in gravistimulation takes place by gravity-perceiving cells called statocytes which results in the formation of a biochemical signal that is transferred to a site of that organ where a curvature response is initiated. The best hypothesized Cholodny-Went model of tropism described that gravistimulation promotes the lateral transport of auxin across various specific gravistimulated organs, with its accumulation at the basal region. The consequence of such distribution of auxin leads to curvature of shoot upwards and root downwards (Blancaflor and Masson, 2003; Robyn et al., 2005). The fountain model of auxin transport in roots postulated that auxin is transported through the vasculature into the root cap (Wolverton et al., 2002). In Arabidopsis roots, AUX1 and members of the LAX family of putative transmembrane transporters facilitate auxin influx within cells (Bennett et al., 1996; Robyn et al., 2005). Auxin influx transporters, auxin efflux PIN family of transporter protein stability and action can be regulated at several 
stages, such as their transcription, stability, subcellular dynamics and polar targeting as well as PIN transport. During gravistimulation the PIN3 auxin transporter relocalization occurs from a symmetric distribution towards the newly established bottom side in roots. The flow of auxin towards the root tip is also redirected to the direction of gravity vector, and further transportation of auxin occurs towards the elongation zone by epidermal PIN2/AUX1-mediated flow. At the lower side of the elongation zone, inhibition of cellular growth occurs which inturn causes the downward bending of the root. (Figure 9); (Friml, 2010).

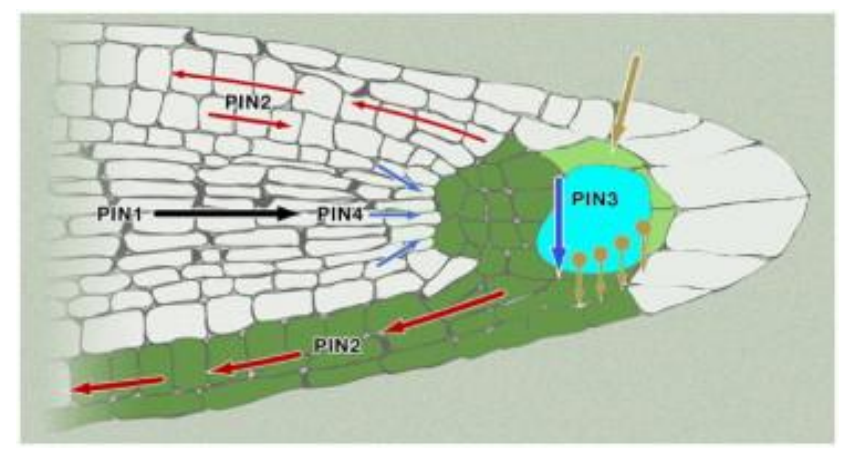

Figure 9: Auxin transport during root gravitropic response in root. Auxin accumulation is depicted by green; arrows (PIN1, black; PIN2, red; PIN4, light blue; PIN3, brown) depict auxin flow mediated by a particular transporter; the dark blue arrow indicates the gravity vector and the direction of bending (Friml, 2010).

Auxin itself can act as a feedback to regulate its own dynamics at multiple levels including PIN transcription (Vieten et al., 2005; Friml, 2010) It was reported that mutations within PIN2 gene confer an auxin-sensitive phenotype which is supported with the loss of auxin efflux carrier function in root cells. In such cases exogenous auxin will accumulate within elongating root cells in the absence of an auxin efflux carrier, resulting in an auxin-sensitive phenotype and subsequently causing inhibition of root growth and development (Müller et al., 1998). During early phases of gravity signal transduction the PIN3 localization plays a pivotal role, typically localizing symmetrically towards the plasma membrane of statocytes of the root cap columella, and then relocalization occurs quickly to basal membranes upon gravistimulation (Friml et al., 2002). As the PIN protein stability is very important in normal auxin transport in roots, so one of the factors that is the phosphorylation status of PIN transporter can be another controlling mechanism how PIN polarity governs root gravitropism. Previously it was reported that inactivation of the AtCRK5 gene by a T-DNA insertion resulted in reduction of primary root elongation and delayed gravitropic response. Since this kinase phosphorylated in vitro the hydrophilic loop of PIN2, this delayed phenotype of the AtCRK5 protein kinase mutant was explained 
to be the consequence of decreased PIN2 auxin efflux transporter levels from apical membranes of epidermal cells (Rigó et al., 2013).

\subsection{Photosynthesis and light-induced damage in plants}

Photosynthesis is one of the most efficiently cycled and sustainable processes known in nature involving the conversion of light energy into chemical energy by providing the basic energy source for life on earth and the molecular oxygen. The process of photosynthesis occurs in the chloroplast thylakoid membrane where the various reaction centers and protein complexes of this process are present such as photosystem I (PSI), photosystem II (PSII) and light-harvesting antennae, cytochrome b6f (Cyt b6f), and ATP synthase (Eberhard et al., 2008; Hohmann-Marriott and Blankenship, 2011). Though light is reported to be the main source of energy during the process of photosynthesis, but under the extreme light conditions it can also be damaging in plants. When the level of light exceeds the capacity of photosynthesis in leaves and during excess levels the absorbed energy can potentially inactivate the reaction centers (RCs). Consequently, this excessive level of light can lead to the formation of reactive oxygen species (ROS) and eventually to the reduction of electron transport efficiency and photosynthesis (Powles, 1984; Osmond and Förster, 2008; Tian et al., 2017)

This damage of light induction mainly targets PSII which leads to inactivation of the electron transport with further subsequent oxidative damage to D1 protein of the reaction centre, in particular. This damage in protein and inactivation can be induced by two variable mechanisms, which can arise from the P680 either the acceptor side of it or from its donor side. This damaged version of D1 protein is scheduled for degradation and digestion by around one serine-type proteinase which is tightly associated with the Photosystem II complex. (Aro et al., 1993).

\subsubsection{Reactive oxygen species (ROS)}

In plants the aerobic metabolic processes takes place continuously involving the mode of respiration and photosynthesis. These continuous metabolically active processes like the activity of photosynthetic and mitochondrial electron transport lead to the production of various reactive oxygen species in different organelles of cells like mitochondria, chloroplasts, and peroxisomes (Apel and Hirt, 2004). The ROS species comprises of free radical forms like superoxide radicals $\left(\mathrm{O}_{2}{ }^{-}\right)$, hydroxyl radical $(\mathrm{OH})$, perhydroxy radical $\left(\mathrm{HO}_{2}\right)^{\circ}$, and alkoxy radicals $(\mathrm{RO})$, and non-radical (molecular) forms hydrogen peroxide $\left(\mathrm{H}_{2} \mathrm{O}_{2}\right)$, and singlet oxygen $\left({ }^{1} \mathrm{O}_{2}\right)$; (Gill and Tuteja, 2008). The 
highly reactive nature of ROS and their acquisition causes oxidation of proteins, lipid peroxidation and damage to nucleic acids, finally destined to cell death (Driscoll et al., 2006). However ROS production in cells has also been reported to regulate gene expression and post-translational modifications of proteins displaying its role in signal transduction network. This duality of ROS activity is under strict control of the defined antioxidant system, which maintains the ROS homeostasis in plants (Pucciariello et al., 2012). This property of ROS which enables them to act as toxic agents or they are observed to regulate various biomolecules based on their concentration. This regulated balance maintenance between ROS production and scavenging/antioxidants is very important for stable growth and development of a plant. In fact this activity of ROS modulations and maintenance of homeostasis is in tight regulation inside the cell (Mittler et al., 2004; Martin et al., 2013).

Various ROS species in plants such as ${ }^{1} \mathrm{O}_{2}, \mathrm{O}_{2}{ }^{-{ }^{-}}$and $\mathrm{OH}^{\cdot}$ posses very short half-life and diffuse over near distances, predicting their importance in signal transduction (Pitzschke et al., 2006). Along with these ROS, the other species which is very important is $\mathrm{H}_{2} \mathrm{O}_{2}$, which can act as a secondary messenger in plant cells during signaling cascade (Choudhury et al., 2013). This ROS is the most stable with the longest half-life and is reported to cross plant membranes through aquaporins, with its analogous physical features with water molecule (Bienert et al., 2006). ROS species are produced continuously in plants as the byproducts of aerobic metabolism. Plants are deluged with mechanisms to tackle the increased levels of ROS during abiotic stress conditions, and usually the ROS generation can be purposefully generated as signaling molecules to control the various processes involving the pathogen defense, programmed cell death, and stomatal behavior. Novel insights into the complexity and roles of ROS in plants evolved from the genetic analyses of ROS detoxifying and signaling mutants (Apel and Hirt, 2004).

\subsubsection{Singlet oxygen $\left({ }^{1} \mathrm{O}_{2}\right)$ as one of the potent $\mathrm{ROS}$ species in cell death}

Singlet oxygen is the most reactive form of molecular oxygen, which is not a free radical form, but a toxic form of oxygen leading to damage in chloroplast membranes by promotion of oxidation of unsaturated fatty acids. It displays a contributing role in signaling and due to its high reactivity leading to cell death (Halliwell, 2006). Singlet oxygen is one of the ROS species that is generated during various biochemical reactions in plants. Its production is tightly light-dependent and sometimes get accumulated in the photosynthetic regions of plants during various stress conditions. It is highly electrophile in 
nature, and can undergo various reactions in biological molecules. It is also reported to play a very important role in cellular stress by the inducing toxicity or its signal to initiate death program in final cellular death (Koh et al., 2016).

Recently it was followed that the effects on the growth of roots in Arabidopsis was highly correlated with the appearance of singlet oxygen, when detected using the singlet oxygen-specific probe singlet oxygen sensor green (SOSG) (Chen and Fluhr, 2018). This appearance of singlet oxygen was followed by cellular death with the detected loss of membrane integrity. These findings suggested a light-independent, type-I source of singlet oxygen production in Arabidopsis displaying an essential role of singlet oxygen in conveying the root response to osmotic stress (Chen and Fluhr, 2018). Singlet oxygen production is typically associated with inefficiency in the disappearance of energy of photosynthesis or can come from light reactions as a result of accumulating the chlorophyll precursors as observed in fluorescent $(f l u)$-like mutants (Mor et al., 2014). Singlet oxygen production occurs from various stress pathways and can emerge from compartments other than the chloroplast in a light-independent manner. Contribution to the role of singlet oxygen in regulation of plant stress and response is more ubiquitous. Singlet oxygen appears to play a role in retrograde signaling from the chloroplast to the nucleus as established by various mutants of FLUORESCENT (FLU), and EXECUTER1 (EX1) and EX2 (Mor et al., 2014). Under various stress and excess light conditions singlet oxygen is believed to be quite destructive and leads to ROS-induced cell death in leaf tissues (Triantaphylidès et al., 2008). Isolation and characterization of the flu mutant in Arabidopsis, was observed to cause a drastic defect in nuclear-encoded FLU protein which is involved in the negative feedback mechanism of the $\mathrm{Mg}^{2+}$ branch of tetrapyrrole biosynthesis (Meskauskiene et al., 2001; Meskauskiene and Apel, 2002). The isolation and initial characterization of $f l u$ mutant was conducted during a genetic screen for etiolated mutant seedlings that lost the capability to limit the accumulation of protochlorophyllide (Pchlide) in the dark and were shown to emit strong red Pchlide fluorescence in blue light. In case of etiolated seedlings, introduction of exl mutation was not reported to suppress the harmful effect of the flu mutation (Laloi and Havaux, 2015). Perhaps this incapability of this mutation in exl under such conditions is credited to fact that in the $f l u$ and $f l u$ exl etiolated seedlings the Pchlide build up is about four times higher than seedlings grown in continuous light and transferred to the dark conditions for 8 hours. These correspond to the earlier light conditions in which exl mutation was shown to curb cell death and stop growth. (Wagner et al., 2004; Przybyla et al., 2008; Laloi and Havaux, 2015). 


\section{AIM AND OBJECTIVES:}

Our aim was to characterize functionally the AtCRK protein kinase members as until now there was only very limited information available about their localization and functional role during Arabidosis thaliana growth and development. Our main objectives in this study included as follows:

- Identification and acquisition of Atcrk T-DNA insertion mutants from public mutant, collection and characterization of these mutant lines to generate homozygous lines and test the expression of their mutated genes (genotyping process).

- Phenotyping of the selected homozygous Atcrk mutants and performing the germination and root growth assays, also testing their root/hypocotyl gravitropic bending capability under gravistimulation.

- To report the subcellular localization of cDNA cloned version of 35S::AtCRKeGFP tagged proteins initially by the protoplast transient expression assay and finally in the roots of transgenic transformed seedlings.

- Characterization of the potential role of AtCRK1 protein kinase in response to its sensitivity to continuous light stress involving the regulation of ${ }^{1} \mathrm{O}_{2}$-triggered cell death and maintenance of cellular redox homeostasis.

- Characterization of the role of AtCRK5 protein kinase during embryogenesis by the network of auxin-gibberellic acid hormonal crosstalk through a cooperative role of auxin efflux carriers PIN1, PIN4, PIN7 and influx carrier AUX1. 


\section{MATERIALS AND METHODS:}

\subsection{Growth conditions and plant materials}

Arabidopsis thaliana (L.) Columbia-0 ecotype (Col-0) was used as a wild type control in all the experiments. Seeds were sterilized and kept for two days at $4{ }^{\circ} \mathrm{C}$ for stratification, then transferred on plates containing half-strength Murashige and Skoog culture medium 1/2MS (Duchefa, Haarlem, Netherlands) containing 2.2g/l MS salt supplemented with B5 vitamins, $0.5 \%$ sucrose (Molar Chemicals Kft, Hungary) and $0.8 \%$ phytoagar (Duchefa, Haarlem, Netherlands). The plates were placed vertically for root growth and gravitropic assay and incubated in thermostat room either under short-day conditions (SD, 8h light/16h dark cycle, $22{ }^{\circ} \mathrm{C}, 100 \mu$ mole photons $\mathrm{m}^{-2} \mathrm{~s}^{-1}$ light intensity) or under continuous light (CL, $22{ }^{\circ} \mathrm{C}, 50 \mu$ mole photons $\mathrm{m}^{-2} \mathrm{~s}^{-1}$ light intensity). The Atcrk5-1 mutant has been described previously (Rigó et al, 2013; Baba et al., 2018). The transformed plants of Col-0 and Atcrk5-1 containing the constructs like DR5::GFP (auxin-responsive reporter), AtCRK5GFP, AUX1::YFP, PIN1-GFP, PIN3-GFP, PIN4-GFP and PIN7-GFP were also already described earlier in Rigó et al, 2013.

\subsubsection{Seed/embryo size determination and embryo isolation}

For the determination of seed/embryo size the wild type Col-0 and Atcrk5- 1 seeds were sterilized in standard manner and stored in vials for 2 days at $4{ }^{\circ} \mathrm{C} .100 \mathrm{ul}$ seeds from each vial were taken out on slide and covered with cover slips. Then photographs were taken with same microscopic parameters using stereo microscope (Nikon SMZ800, Japan) attached with a CCD camera (Nikon Cool pix 995, Japan). Seed size (perimeter) in intact seeds was quantified in the wild type Col-0 and mutant Atcrk5-1 after the imbibition of seeds for just 2 days in water at $4{ }^{\circ} \mathrm{C}$ and then photographing the seeds by the stereo microscope (Nikon SMZ800, Japan) attached with CCD camera (Nikon Cool pix 995, Japan) finally the perimeter of seeds was measured by ImageJ software (National Institute of Health, USA). The average values are from minimum 100 independent seeds. Length of embryo axis were measured from shoot apical meristem (SAM) untill root apical meristem (RAM), calculated in walking stick embryos stage arised from the previously described experiment. At least the axis measurement was performed on 100 bent cotyledon embryos by ImageJ. The differences in the hypocotyl hook angles of wild type Col-0 and mutant Atcrk5-1 embryos was performed after just 2 days imbibition at $4{ }^{\circ} \mathrm{C}$ and after it the bent stage embryos were carefully pressed out from the seed coat by forceps under 
stereomicroscope (Nikon SMZ800, Japan) and then photographed by CCD camera (Nikon Cool pix 995, Japan). Images were evaluated by ImageJ software.

\subsection{Genotyping and transcript analysis of the T-DNA insertion mutants}

All the T-DNA insertion mutants of AtCRK family members were ordered from Salk Institute (SIGnAl website): Atcrk1-1 (SALK-071004), Atcrk1-3 (SALK-037483), Atcrk2-1 (SALK-080050), Atcrk2-6 (SALK-090938), Atcrk3-1 (SALK-142932), Atcrk4-1, (SALK-028536), Atcrk4-2 (SALK-009503), Atcrk8-1 (SALK-079502). The mutant used for Atcrk1-1 (SALK-071004) was the same one which was used in earlier studies (Liu et al., 2008; Tao and Lu, 2013). The presence of T-DNA insertions in AtCRK6 and AtCRK7 genes could not be confirmed and they were not used for experimental studies. Finally the genotyping of all the ordered mutants was performed and further studied for gene expression level by qRT-PCR to verify the knock out status of mutants. According to transcript analysis of these mutants Atcrk1-1, Atcrk2-1, Atcrk2-6, Atcrk3-1 and Atcrk8-1 were reported to be full knockout (KO) mutants; while Atcrkl-3 was knock down (KD), Atcrk4-1 and Atcrk4-2 were strong knock down mutants. The homozygous mutant lines were used for further analysis and experimental studies. Relative expression of AtCRK genes in different parts was evaluated in 14 days old wild-type (Col-0) Arabidopsis plants grown in vitro at short-day (SD) and continuous light (CL) conditions by qRT-PCR. Relative transcipt levels of these genes were standardized to glyceraldehyde-3-phosphate dehydrogenase (GAPDH2); (Czechowski et al., 2005). Primers pairs can be found in Supplementary Figure S1, and were designed by Primer3Plus software.

\subsection{Phenotyping of the T-DNA insertion mutants}

\subsubsection{Germination and root growth assay}

Germination assay of the wild type Col-0 and mutant seeds of the similar age was carried out on $1 / 2 \mathrm{MS}$ medium in short day light conditions (SD, 8h light/16h dark cycle, 100 $\mu$ mole photons $\mathrm{m}^{-2} \mathrm{~s}^{-1}$ light intensity) at $22{ }^{\circ} \mathrm{C}$ and then the rates of cotyledon opening was monitored for four continuous days. The measurements of root growth was carried out on $1 / 2 \mathrm{MS}$ medium under continuous light conditions (CL, $50 \mu$ mole photons $\mathrm{m}^{-2} \mathrm{~s}^{-1}$ light intensity) at $22{ }^{\circ} \mathrm{C}$. First the seeds were sterilized and stratified for two days at $4{ }^{\circ} \mathrm{C}$ and then the seeds were germinated in vertical position. Seedling roots were labelled in every 
24 hours and the root growth rates of Col-0 and Atcrk mutants were measured from $5^{\text {th }}$ day till $11^{\text {th }}$ day. Then the scanned seedling images were evaluated by ImageJ software.

\subsubsection{Root and hypocotyl gravitropic bending assay}

Root gravitropic assay was performed under continuous light in the thermostat room. Six-day-old, vertically grown seedlings were gravistimulated by changing orientation of plates by $135^{\circ}$, and angle of root bending was recorded 24 hours after reorientation by scanning the pertri dishes. The rate of root bending was determined by measuring the angle between the growth direction of root tip and horizontal baseline. In case of hypocotyl bending response, six-day-old seedlings with initial 6 hours of light induction were germinated in dark at vertical position and plates were reoriented 5 days after germination. Hypocotyl bending angles were measured after 48 hours of reorientation with Image J software. Around 50 seedlings of wildtype Col-0 and Atcrk mutant seedlings were used for each root and hypocotyl bending test, in two biologically independent experiments.

\subsubsection{Fresh weight, chlorophyll and carotenoid content measurement}

For chlorophyll determination, the rosette material of different tones was collected, and their fresh weights were determined and then extracted with $95 \%$ ethanol at $4{ }^{\circ} \mathrm{C}$, overnight. Finally chlorophyll content was determined by measuring absorbances at 470 , 648, and $664 \mathrm{~nm}$ using Multiskan G0 spectrophotometer (Thermo Fischer Scientific, Vantaa, Finland). Absorbance was measured between OD 0.3 and 0.8. Chlorophyll and carotenoid concentrations were then calculated with equations as described in (Lichtenthaler and Buschmann, 2001). For fresh weight determination, rosettes of three seedlings were measured in five repeats. Two independent biological repetitions were performed with similar results.

\subsection{Plant transformation and GFP localization studies}

\subsubsection{Gene cloning and plant transformation}

Full length CRK cDNAs were amplified with PCR from various Arabidopsis cDNA library by Phusion High-Fidelity DNA Polymerase (Thermo Fischer Scientific, Vilnius, Lithuania) using gene specific primers (Supplemental Figure S1) and then cloning the fragment into pENTR2b Gateway vector. Error free cDNA clones were further used to get $35 \mathrm{~S}:: \mathrm{cCRKs}-$ GFP gene fusions by cloning full length AtCRK cDNAs into pK7FWG2.0 expression 
vector (Karimi et al., 2002) using LR Clonase ${ }^{\mathrm{TM}}$ II enzyme (Thermo Fischer Scientific, Vilnius, Lithuania). Final clones were tested by sequencing and were transformed into plants by Agrobacterium-mediated transformation using the GV3101/pMP90 strain (Koncz et al., 1994). Col-0 wild type Arabidopsis plants were transformed using in planta infiltration (Bechtold et al., 1993). Primary transformants (T1) plants were selected on 1/2 MS medium containing $30 \mathrm{mg} \mathrm{l}^{-1}$ kanamycin. T3 generation homozygous lines of plants were used for CRK-eGFP localization experiments. The cDNA cloned versions of AtCRKs were used for the subcellular localization by the protoplast transient expression and later in the plant roots. In the figure below (Figure 10) we show the schematic map of the construct used for cloning of all AtCRK genes. The in-frame fusion of AtCRK proteins with green fluorescent protein (GFP) at C-terminal was generated by replacing the stop codon with coding regions of green fluorescent protein and the pCaMV35S promoter was added at the $\mathrm{N}$-terminal region.

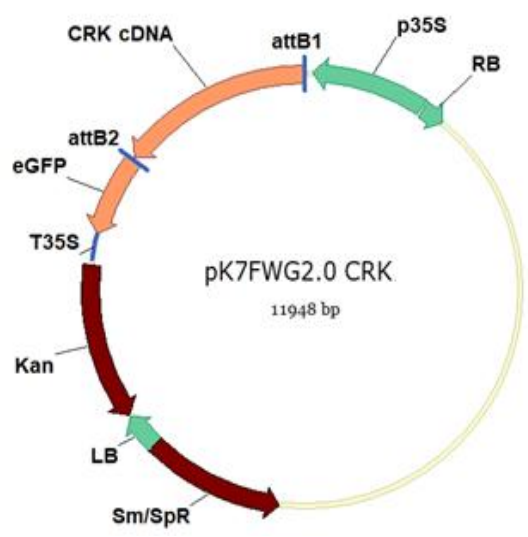

Figure 10: Schematic map of 35S::cCRKs-GFP vector construct. Fusion of GFP at the C-terminal and pCaMV35S promoter at N-terminal of amplified cDNA fragment, this vector was used further for creating GFP construct for rest AtCRKs.

\subsubsection{Protoplast transformation studies}

Intracellular localization of AtCRK-eGFP fusion protein was first initiated in protoplasts isolated from Arabidopsis thaliana suspension cultures and then the polyethylene glycol mediated (PEG) transformation of AtCRKs-eGFP constructs was performed with transient expression studies using the method described in (Rigó et al., 2008). Finally the GFP localization studies were carried by confocal laser scanning microscopy (CLSM) (Olympus FV1000, Tokyo, Japan) microscopy after $24 \mathrm{hrs}$ of transformation as was described in (Rigó et al., 2013). 


\subsubsection{Hairy root transformation for the 35S::cCRK1-GFP}

Hairy root transformation was performed by CD3-967/pBIN20-Golgi mCherry marker construct (TAIR stock) containing Agrobacterium rhizogenes strain (Arqua-1) propagated on YEB agar plate supplemented with $100 \mathrm{mg}^{-1}$ kanamycin at $28^{\circ} \mathrm{C}$. Hairy root transformation was performed according to the protocol of (Ron et al., 2014). Briefly, Arabidopsis thaliana Col-0 plants expressing 35S::cCRK1-GFP construct were propagated on $1 / 2$ MS media as indicated above and then five days old plants were used for transformation. Inoculation of CD3-967/pBIN20 containing Arqua-1 strain was done into $10 \mathrm{ml}$ liquid YEB media supplemented with $100 \mathrm{mg} \mathrm{l}^{-1}$ kanamycin and rotated overnight at $28^{\circ} \mathrm{C}$ in a shaker (200 rpm). Agrobacteria were then centrifuged and resuspended in $1 / 2 \mathrm{MS}$ liquid medium following the immersion of Arabidopsis plants in this solution for 2-5 minutes. After two days of co-incubation in dark, plants were transferred onto $1 / 2$ MS plates with $200 \mathrm{mg} \mathrm{l}^{-1}$ cefotaxime (Duchefa, Harleem, Netherlands) and $200 \mathrm{mg}^{-1}$ carbenicillin (Duchefa, Harleem, Netherlands) and cultured at $22^{\circ} \mathrm{C}$. After three-four weeks, the newly formed hairy roots were used for microscopic studies.

\subsection{Physiological characterization of the Arabidopsis CRKs}

\subsubsection{PSII photochemical activity measurements}

PSII Photochemical activity measurements were performed on Col-0 wild type as control and Atcrk mutant seedlings which were grown on vertical plates containing $1 / 2$ MS and grown for two weeks in continuous light. The measurement of chlorophyll fluorescence was performed by MAXI-version of Imaging-PAM (Walz, Effeltrich, Germany). After dark adaptation of seedlings for $15 \mathrm{~min}$, minimum and maximum fluorescence yields were measured before and after a saturating pulse, respectively. The seedlings were exposed to seven intensities of photosynthetic active radiation (PAR) increasing from 0 to $281 \mu$ mol $\mathrm{m}^{-2} \mathrm{~s}^{-1}$. Each illuminating period was about $10 \mathrm{~s}$ to ensure the minimum fluorescence in actinic light (Fs) and was followed by an excitation pulse to yield the maximum fluorescence in actinic light (Fm'). The effective PSII quantum yield ( $\left.\Phi_{\mathrm{PSII}}\right)$ was calculated by the Imaging Win software as $\Phi_{\mathrm{PSIl}}=\left(\mathrm{F}_{\mathrm{m}}{ }^{\prime}-\mathrm{F}_{\mathrm{s}}\right) / \mathrm{F}_{\mathrm{m}}{ }^{\prime}$ as described earlier by (Genty, 1988). The kinetics of non-photochemical quenching (NPQ) were calculated as NPQ $=\left(F_{m}-F_{m}{ }^{\prime}\right) / F_{m}{ }^{\prime}$. Kinetics of effective PSII quantum yield $\left(\Phi_{\text {PSIl }}\right)$, ETR and NPQ were obtained by averaging data from fifteen corresponding areas of interest in five 
different seedlings. The presented results are average of three different independent biological repeats.

\subsubsection{Detection of ${ }^{1} \mathrm{O}_{2}$ production}

Singlet oxygen $\left({ }^{1} \mathrm{O}_{2}\right)$ levels were detected by using Singlet Oxygen Sensor Green (SOSG) reagent (Molecular Probes Inc., Eugene, OR, USA) in detached leaves of Col-0 wild type and Atcrk1-1 mutant plants (Flors et al., 2006; Hideg, 2008; Rehman et al., 2016) grown in continuous light on $1 / 2$ MS media. Seedlings were first immersed in $250 \mu \mathrm{M}$ SOSG (dissolved in water) and illuminated with $2300 \mu$ mole photons $\mathrm{m}^{-2} \mathrm{~s}^{-1}$ intensity visible light for $5 \mathrm{~min}$. Leaf samples of infiltrated SOSG were fixed on cover slip with thin layer of agar $(0.8 \%)$. Visualization of green fluorescence was performed by Olympus FV1000 confocal microscope (Tokyo, Japan) using excitation at $504 \mathrm{~nm}$ and emission detection at $525 \mathrm{~nm}$. Images were obtained with a transmitted light detection module with $488 \mathrm{~nm}$ excitation using a laser diode (LD). Simultaneously, chlorophyll fluorescence was also visualized using excitation by the $488 \mathrm{~nm}$ argon laser and fluorescence detection through a $650-750 \mathrm{~nm}$ filter.

\subsubsection{Determination of cell death}

The determination of cell death of Atcrk1-1 mutant and wild type Col-0 seedlings was estimated by using Evans blue staining as described in (Shumbe et al., 2016). Threeweek-old vertically grown seedlings on $1 / 2$ MS media in continuous light conditions and parallelly also in short-day conditions were used and then were stained with $0.1 \%(\mathrm{w} / \mathrm{v})$ Evans blue dye using vacuum infiltration. Then leaves were washed three times for 10 minutes with $0.05 \%$ Tween 20 dissolved in $150 \mathrm{mM} \mathrm{NaCl}$ to remove unbound dye, while the chlorophyll was removed by $96 \%$ ethanol washing. Seedlings were then scanned on white surface for visualizing the dye retention.

\subsection{Microsomal membrane preparation, differential centrifugation and immunoblotting}

To study CRK-GFP localization in transgenic seedlings expressing the 35S::cCRK1eGFP construct the differential centrifugation was performed. Five grams of fresh plant material were homogenized in $10 \mathrm{ml}$ of ice cold extraction buffer $[50 \mathrm{mM}$ Tris- $\mathrm{HCl} \mathrm{pH}$.: 7.5, 1mM Ethylenediaminetetraacetic acid (EDTA) pH.:8.0, $350 \mathrm{mM}$ sucrose, 1-2 mM phenylmethylsulfonyl fluoride (PMSF), protease inhibitor cocktail (1xPIC) (Sigma P9599 
Aldrich,St. Louis,MO,USA), 1mM Dithiothreitol (DTT)]. Filtration through Miracloth (Merck Millipore, Burlington, MA, USA) was perfomed and then the filtered samples were centrifuged by $1000 \mathrm{~g}$ for 5 minutes to remove cell debris. Then the supernatant was collected into new centrifuge tubes. $200 \mu 1$ supernatant was taken into microcentrifuge tube on ice which represents the total extract (total). This extract was centrifuged with $5000 \mathrm{~g}$ for 10 minutes and the supernatant was transferred into new centrifuge tube again. Resuspension of the pellet was done in $1 \mathrm{ml}$ extraction buffer and then pipetted into $1.5 \mathrm{ml}$ microcentrifuge tube. The pellet representing plastids and nuclei was collected again at $5000 \mathrm{~g}$ using cooled table top centrifuge. Then the $200 \mu \mathrm{l}$ from the supernatant was also saved, this was the $5000 \mathrm{~g}$ supernatant (soluble A). After repetition of this step at higher centrifugation $15000 \mathrm{~g}$ for 15 minutes, we could obtain mitochondria, part of the endomembrane and soluble B fractions. Finally centrifugation with $48000 \mathrm{~g}$ for one hour resulted in cytoplasmic and microsomal (all endomembrane and plasma membrane) fractions. Protein concentration of all samples was determined using Bradford reagent (Bio-Rad Laboratories, Hercules, CA, USA). Equal amount of protein i.e, $25 \mu \mathrm{g}$ total protein from each sample was size separated on $8 \%$ SDS-PAGE, transferred onto Immobilon PVDF Membrane (Merck Millipore, Burlington, MA, USA), incubated for one hour in 1xTBST blocking buffer [50 mM Tris- $\mathrm{HCl}(\mathrm{pH} 8.0), 150 \mathrm{mM} \mathrm{NaCl}, 0.05 \%$ Tween20, 5\% dry skimmed milk] and 1.5 h with anti-GFP antibody (Roche, 1:2000 dilution) in blocking buffer. After this treatment, three times washing was done with 1xTBST for 10 minutes, the membranes were incubated for 1.5 hour with an anti-mouse-POD secondary antibody (Pierce, dilution 1:5000), washed with 1xTBST as before, and then overlaid with Immobilon Western Chemiluminescent HRP Substrate (Millipore) and final detection of 35S::cCRK1-GFP was performed by autoradiography.

For the various other GFP expressing plants of AtCRK family, 35S::cCRK2-GFP, 35S::cCRK3-GFP， 35S::cCRK4-GFP， 35S::cCRK5-GFP， 35S::cCRK7-GFP and 35S::cCRK8-GFP expressing plants, differential centrifugation was performed as described (Abas and Lusching, 2010). The frozen $100 \mathrm{mg}$ plant material ground in liquid nitrogen and then was resuspended in ice cold (350 $\mu \mathrm{L} / 100 \mathrm{mg}$ fresh weight) extraction buffer (50 mM MOPS-KOH pH.:7.5, 5\% glycerol, $810 \mathrm{mM}$ sucrose, $10 \mathrm{mM}$ EDTA pH.:8.0, 5 mM EGTA pH.:8.0, 1-2 mM PMSF, 1-2 × PIC-Sigma P9599, 1mM DTT). After this the samples were centrifuged by $600 \times g$ for 4 min for the removal of cell debris, then filtering the supernatant through Miracloth, following the centrifugation again at $(600 \times g, 4 \mathrm{~min})$ to remove cell debris. Then the supernatant was collected and diluted with 
sterile water (1:1). A $50 \mu \mathrm{L}$ aliquot was saved as a total extract (T) and following the rest of the extract was centrifuged at $4^{\circ} \mathrm{C}(21,130 \times g$ for $1.5 \mathrm{~h}$ in eppendorf $5424 \mathrm{R}$ centrifuge). Supernatant was collected into a new tube and this represents the soluble fraction or cytoplasmic (Cy) fraction, and the pellet is the microsomal (Mi) fraction. This microsomal pellet was then washed with membrane wash buffer $(50 \mathrm{mM}$ Tris- $\mathrm{HCl}$ pH.:7.5, $5 \mathrm{mM}$ EDTA pH.:8.0, 5 mM EGTA pH.:8.0, 1-2 × PIC-Sigma P9599, 1 mM DTT) and then the centrifugation was performed for $50 \mathrm{~min}$ at $21,130 \times g$ at $4{ }^{\circ} \mathrm{C}$. Supernatant was finally discarded and then the detection of the various AtCRK-eGFP fusion proteins was performed as described above by a western blotting.

\subsection{RNA isolation and quantitative real time PCR (qRT-PCR)}

\subsubsection{RNA isolation and $q R T-P C R$ from seedlings}

RNA isolation was performed from $100 \mathrm{mg}$ material of either Arabidopsis leaves/roots for AtCRK gene expression in wild type plants and also from whole seedlings to check out the expression level /transcript amount of AtCRK genes in the T-DNA inserton mutants. The isolation was performed by Nucleospin Plant RNA kit (Macherey-Nagel, Düren, Germany). Total RNA was DNase-treated with TURBO DNA-free ${ }^{\mathrm{TM}}$ Kit (Invitrogen by Thermo Fisher Scientific, Vilinus, Lithuania). First-strand cDNA synthesis of $1 \mu \mathrm{g}$ of total RNA was carried out in $20 \mu \mathrm{l}$ using RevertAid M-MuLV Reverse Transcriptase (Applied Biosystems by Thermo Fischer Scientific, Vilinus, Lithuania), using random hexamers. Real-time PCR was carried out with the ABI 7900 Fast Real Time System (Applied Biosystems, Foster City, CA, USA) with the following protocol: 45 cycles at $95^{\circ} \mathrm{C}$ for $15 \mathrm{~s}$, followed by $60^{\circ} \mathrm{C}$ for $1 \mathrm{~min}$. The normalized relative transcript levels were obtained by the $2^{-\Delta \Delta C t}$ method (Czechowski et al., 2005). The specificity of the amplifications of the genes was finally verified at the end of the PCR run through use of the ABI SDS software (Applied Biosystems, Foster City, CA, USA). Reactions were made in three replicates and minimum two to three independent biological repetitions were performed for each gene tested. All set of primers used in this study are listed in Supplementary Figure S1.

\subsubsection{RNA isolation and $q R T-P C R$ from embryos}

Total RNA was isolated from siliques with a slightly modified CTAB-LiCl extraction method (Jaakola et al., 2001). Isolation was performed from $100 \mathrm{mg}$ material collected from greenhouse plants of 45 days old as small (S1-S4), medium (S5-S7) 
siliques for checking gene expression in Col-0 and Atcrk5-1. Ubiquitin-1 was used as an endogenous control in embryo gene expression studies.

\subsection{PIN2 immunolocalization}

PIN2 immunolocalization for roots was performed with wild-type Col-0 and Atcrk mutant seedlings grown vertically on $1 / 2$ MS medium supplemented with $0.5 \%$ sucrose for 7 days in constant light as described in (Friml, 2003).

\subsection{Microscopy}

\subsubsection{Intracellular GFP localization of roots by confocal laser scanning microscope}

For the intracellular localization of GFP fusion in plant roots, seven days old 35S::cCRKs-GFP seedlings grown vertically on $1 / 2 \mathrm{MS}$ media in short day conditions (SD, $8 \mathrm{~h}$ light/16h dark cycle, $22^{\circ} \mathrm{C}, 100 \mu$ mole photons $\mathrm{m}^{-2} \mathrm{~s}^{-1}$ light intensity) and then the roots were imaged using Olympus FV1000 confocal laser scanning microscopy system with IX81 invert microscope and 60x oil N.A 1.35 objective (Tokyo, Japan). Excitation sources were $405 \mathrm{~nm}$ (root cell autofluorescence), $488 \mathrm{~nm}$ (eGFP) and $543 \mathrm{~nm}$ (FM4-64, Mito Tracker Orange, mCherry) lasers. FM4-64 (Invitrogen, Waltham, MA, USA) staining was carried out on 6 days old seedlings by incubating the samples in $5 \mu \mathrm{M}$ FM4-64 for $10 \mathrm{~min}$ for colocalization studies of GFP fluorescence. Spinning disk confocal images were recorded using Visitron spinning disk confocal system (Visitron systems GmBH, Germany) equipped with Yokogawa CSU-W1 spinning disk unit (50 $\mu$ m pinhole diameter), Olympus IX83 inverted microscope (60x oil objective, N.A 1.42), Andor Zyla 4.2 Plus camera, 488 and $561 \mathrm{~nm}$ lasers. Composite images were prepared using the Adobe Photoshop, Adobe Illustrator (Adobe Systems Incorporated, San Jose, CA, USA) and Corel Photopaint (X7) software (Ottawa, Canada).

\subsubsection{Abundance of GFP signal in embryos by LSM microscopy}

Seeds of the GFP tagged lines of wild type Col-0 and Atcrk5-1 plants of various developmental stages were collected into $10 \%$ (v/v) glycerol from siliques. Then embryos were pressed out from seeds cautiously by using a mild pressure with glass cover. Then the abundance of GFP signals in case of various stages of embryos for auxin transporters (PIN1, PIN4, PIN7 and AUX1), also the AtCRK5-GFP localization and the auxin sensor DR5::GFP was analyzed using the Olympus FV1000 confocal laser scanning microscopy 
(Tokyo, Japan). Color-coded heat map was created to compare fluorescence intensity for better visualization of the differences in the intensity of PIN1-GFP. Images were prepared using the Adobe Photoshop and Illustrator softwares (Adobe Systems Incorporated, San Jose, CA, USA).

\subsubsection{Embryo morphology monitoring by Cell-R microscope}

Siliques from each Col-0 and Atcrk5-1 green house plants (S1 to S11/S12 where S1 represents the youngest and S11/S12 the oldest siliques) containing the various developmental stages of embryos were selected. Seeds were dissected from siliques and put into Hoyer's solution (containing Chloral hydrate and glycerol) 4 hours for seeds arising from younger siliques and overnight in case of seeds from older siliques for preclearing (Stangeland and Salehian, 2002). Then the seed developmental stages were determined by differential interference contrast microscopy (Cell-R, Leica, Germany).

\subsection{Total gibberellic acid (GA) measurement by Competitive GA ELISA assay}

In the case of total GA content measurement the septum bearing the seeds without valves originated from the medium sized siliques (S5-S7 from the top of inflorescence) were collected from 6 weeks old green house grown Arabidopsis plants. Seeds were dissected from the siliques under stereo microscope (Nikon SMZ800, Japan) and were collected into eppendorf tubes on dry ice. Samples were fully grinded then using Retsch (Haan, Germany). The grounded samples were incubated overnight at $4^{\circ} \mathrm{C}$ in $10 \mathrm{ml}$ of $80 \%$ methanol solution. After centrifugation $13300 \times g, 10$ minutes at $4^{\circ} \mathrm{C}$, the supernatant was transferred to new tubes and completely lyophilized. We added $200 \mu 1$ PBS to each sample and loaded $50 \mu 1 /$ well. Three technical repetitions were used. For ELISA measurement we followed the protocol of the kit (ELISA Kit for Gibberellic Acid (GA), CEA759Ge, Cloud Clone Corp. Wuhan, China).

\subsection{Cloning of the PIN4 and PIN7 hydrophilic loop (HL)}

For the cloning of the PIN4 and PIN7 hydrophilic loop into the vector pMALp2 from NEB (www.neb.com) we used the larger Hisidine tag containing vector because of the intital problem with solubility of the PIN1 Hydrophilic Loop in smaller tag vector. Firstly we designed specific primer sets (Supplemental Figure S1) to amplify the fragments from various cDNA libraries and furthermore we used the fidelity enzyme Phusion polymerase 
following the manufacture instruction to amplify the fragment. Only in the case of PIN4 HL loop amplification we could get enough PCR products. After the amplification, the gel purification of the PIN4HL loop fragment was done and was digested using EcoRI-SalI enzyme, and ligated into pMALp2 vector to get in frame fusion with $\mathrm{N}$-terminal maltose binding protein (MBP) tag. The error free clones were identified by sequencing and used for protein expression studies. In case of the cloning of the PIN7 HL loop, we had a problem to amplify the HL loop sequence so we tried several types of cDNA sources but were not able to amplify the fragment. Finally we choose alternate way, as the genomic region of PIN7 contains a small intron sequence (yellow highlight) (Supplemental Figure S2), so we designed the primers (Supplemental Figure S1) to eliminate this sequence from the final clone. We used the following Phusion enzyme based high fidelity PCR reactions using Col-0 purified genomic DNA as template for the two reactions.

A1 PCR reaction: P7HL_F_EcoRI_pMALp2+ pin7genomic3' primer pairs, to amplify the 770bp long DNA fragment, which located before the intron sequence.

A2 PCR reaction: P7HL_R_SaLI_pMALp2+ pin7genomic5' primer pairs, to amplify the 268bp long DNA fragment, which located after the intron sequence.

Both of the fragments were gel purified and then assembly was performed by following the Phusion enzyme based high fidelity PCR reaction. Templates isolated fragment from A1 and A2 PCR reaction were 1-1ng from each. PCR primers: P7HL_F_EcoRI_pMALp2 and P7HL_R_SaLI_pMALp2. After the PCR reaction was performed and run on agarose gel electrophoresis and we isolated the 1012bp long PCR product. The isolated PIN7HL fragment was digested with EcoRI-SalI enzyme and ligated into pMALp2 vector to get in frame fusion with N-terminal of Maltose Binding Protein (MBP) tag. The error free clones were identified by sequencing and used for protein expression studies.

\subsection{Protein expression and purification}

The confirmed clones of pMALp2-PIN4HL and pMALp2-PIN7HL were transformed into BL21 (DE3) Rosetta (Novagen) and plated onto LB agar plate supplemented with $34 \mathrm{mg} / 1$ Chloramphenicol, $100 \mathrm{mg} / \mathrm{l}$ Carbenicillin and $0.4-0.5 \%$ glucose. Then the purification of MBP tagged protein was performed by streaking out sesame seed size of bacterial culture and inoculated into the $20-25 \mathrm{ml} \mathrm{LB}$ liquid media supplemented with antibiotics $(34 \mathrm{mg} / 1$ Chloramphenicol, 100mg/l Carbenicillin and 0.4-0.5\% glucose ) and left to grow overnight $37^{\circ} \mathrm{C}$ on rotary shaker. The overnight culture was diluted to $1: 10$ into similar media and 
grown at room temperature or $37^{\circ} \mathrm{C}$ until it reaches the OD600 $=0.7-0.8$. At that time the inducer IPTG was added to the final concentration $0.3 \mathrm{mM}$ and continued to grow on room temperature for additional 4-5 hours. After this the cells were collected by centrifugation and resuspend into $1 / 10$ volume of lysis buffer, $20 \mathrm{mM}$ Tris- $\mathrm{HCl} \mathrm{pH}: 7.4-7.5,200 \mathrm{mM}$ $\mathrm{NaCl}, 1 \mathrm{mM}$ EDTA pH: 8.0, $1 \mathrm{mM}$ DTT. Now we added lysozyme at $1 \mathrm{mg} / \mathrm{ml}$ final concentration, and incubate at room temperature for 10 minutes. And then add $0.5 \%$ Triton X-100 final concentration, $1 \mathrm{mM} \mathrm{MgSO}_{4}$ final concentration, DNase and RNase 5-5 $\mu \mathrm{l}$ for each $5 \mathrm{ml}$ Lysis buffer from $10 \mathrm{mg} / \mathrm{ml}$ stock. Incubate $4^{\circ} \mathrm{C}$ ten minutes, or until the solution viscosity disappear. Centrifuge the lysis mixture using SS34 rotor on $13000 \mathrm{rpm}$ at $4{ }^{\circ} \mathrm{C}$ for 15 minutes. Supernatant was saved and add 200-300 $\mu 1$ settled Amylose-resin (NEB), which equilibrated with lysis buffer. Incubate in cold room on low speed rotary shaker for one hour long. After the end of incubation time wash the matrix with the following solution: $1 \times 5 \mathrm{ml}$ Lysis buffer $0.5 \%$ Triton X-100, 1x5ml Lysis buffer $0.2 \%$ Triton X-100. After this step, you have to pipette the matrix into the empty column e.g, lilac QIAshredder Mini Spin Column from Qiaquick RNA isolation kit, or equivalent column with plastic filter disc inserted into the $2 \mathrm{ml}$ Eppendorf tubes. Elution was done by elution buffer $3 \mathrm{X} 400 \mu \mathrm{l} 10 \mathrm{mM}$ Maltose, $0.2 \%$ Triton X-100 in lysis buffer. After elution samples were kept on ice. From each elution, run 10-10 $\mu 1$ on SDS PAGE as before. After staining you we choose the fraction which contains most of the fusion protein. Then the collected samples were dialyzed overnight in the following solution in the cold room: $50 \mathrm{mM} \mathrm{NaCl}, 10 \mathrm{mM}$ Tri-HCl pH: 7.5, 10\% glycerine, 5mM B-mercapthoethanol. The dialyzed samples were aliquot and store $-80^{\circ} \mathrm{C}$ until use.

\subsection{In vitro kinase assay and Mass spectrometry}

For mass spectrometry assay, we are using about 1-1 microgram of purified His $_{6}$-CRK5 kinase (purified by Dr. Gabor Rigo for previous studies) and MBP-PIN4HL or MBPPIN7HL as a substrate. We performed in vitro kinase assay in $20 \mu l$ kinase buffer $(20 \mathrm{mM}$ Tris-HCl [pH 8.0], 5mM MgCl2, 1mM DTT) supplemented with 1mM ATP (Thermo Scientific), then incubated in room temperature for 45-60 min. The reaction was stopped by adding $1 \mathrm{X}$ Laemmli SDS sample buffer, boiled then size separated. After staining with Coomassie dye, the MBP-PIN4HL or MBP-PIN7HL stained protein band were excised and analyzed by mass spectrometry in the Laboratory of Proteomics Research of the BRC (http://www.brc.hu/core_proteomics_research.php). A small aliquote of the tryptic digests was retained and the rest subjected to phosphopeptide enrichment by immobilized metal 
affinity chromatography on Fe-NTA magnetic agarose beads. LC-MS/MS analysis of the digests with and without phosphopeptide enrichment was performed on a Thermo LTQ Orbitrap Fusion Lumos mass spectrometer in data dependent mode applying HCD fragmentation followed by database search against the sequences of the fusion proteins appended to E.coli entries in the Uniprot.2019.6.12 database. Results of the database searches were manually validated.

\subsection{Bioinformatic analysis and tools}

Primers designed for the genes investigated in this study were constructed using Primer3Plus software (http://www.bioinformatics.nl/cgi-bin/primer3plus/primer3plus.cgi). For DNA manipulation VectorNTI (ThermoFisherScientific,Waltham, MA, USA) and Lasergene (DNAStar Inc., Madison, WI, USA) program suits were used. ABI SDS software (Applied Biosystems, Foster City, CA, USA) was used to analyze the specificity of the amplifications of the genes for expression by qRT-PCR. Phylogenetic tree was constructed by using MEGA5.05 software (http://www.megasoftware.net/software). During phylogeny reconstruction, neighbour-joining bootstrap method with 500 bootstrap replications was used in this construction. Root growth rate, hypocotyl/root gravity bending and embryo size/hook bending were all measured by ImageJ software (https://imagej.net/Fiji/Downloads).

\subsection{Statistical analysis}

The experiments were carried out atleast with minimum two or three independent biological repetitions. Data are presented as the mean \pm standard error (SE) calculated from the combined data of biological repetitions. Differences from wild type control were determined by Student's t-test and the significant differences were represented as follows: * $\mathrm{p} \leq 0.05 ; * * \mathrm{p} \leq 0.01 ; * * * \mathrm{p} \leq 0.001$. Two-way ANOVA test was used for the statistical analysis of photosynthetic parameters measurements and was performed using the OriginPro 8.6 software. 


\section{RESULTS}

\subsection{Phylogenetic analysis of $\underline{C D P K-\underline{R} e l a t e d} \underline{\text { Kinase }}$ (AtCRK) family in Arabidopsis thaliana}

Eight of the CDPK-related kinase (AtCRK1-AtCRK8) genes were reported to encode proteins with high degree of sequence similarity. Analysis of their phylogenetic tree displayed a pairwise close relationships between various AtCRK genes like; AtCRK1 and AtCRK7, AtCRK4 and AtCRK6, AtCRK2 and AtCRK8 (Figure 11).

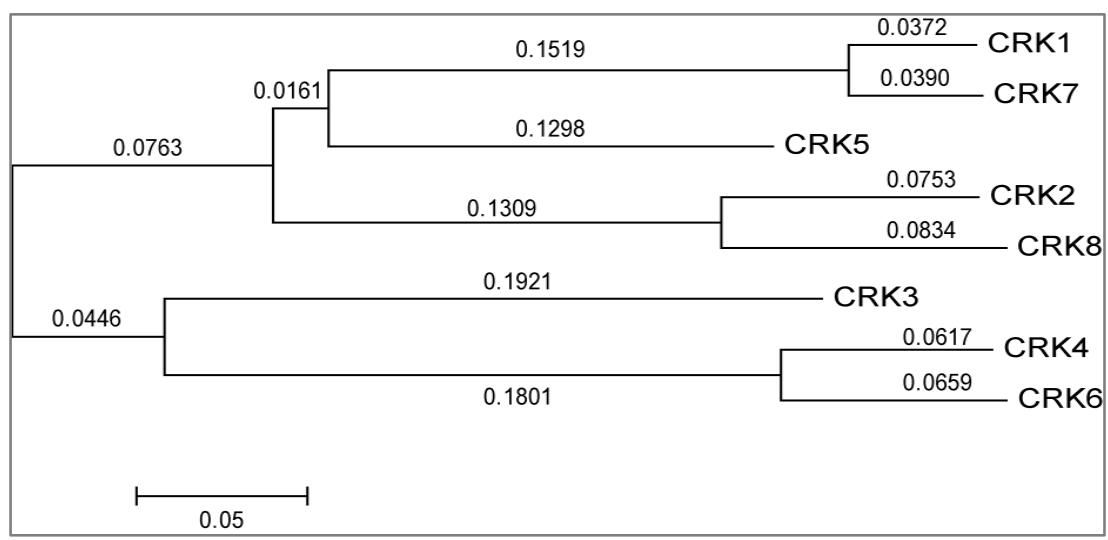

Figure 11: Phylogenetic tree of AtCRK proteins. Bootstrap values are indicated. During phylogeny reconstruction, we used neighbour-joining bootstrap method with 500 bootstrap replications.

\subsection{Expression analysis of AtCRK genes in variable light conditions}

All the AtCRKs displayed a considerable variation in their expression levels of the genes in their specific organs tested. By using the public transcript analysis profiling data (eFP Browser, See Supplemental Figure S3/S4) (Winter et al., 2007) we could correlate that there is a considerable difference between expression levels of these genes and organ specificities of these genes. Based on this browser data among all the genes studied, AtCRK5 had the highest levels of expression when compared with other genes, while AtCRK6 expression was on the limit of detection. This above dataset from eFP Browser available online reported that moderate effect was observed in the gene expression studies of AtCRK genes on any external stimulus. No strong effect of light on AtCRKs expression was observed; in fact expression was found to be slightly enhanced in AtCRK1, AtCRK3 and AtCRK5, while in genes AtCRK2, AtCRK4 and AtCRK7 the expression was seen reduced by light. When the transcript levels were documented in specific parts of rosettes and roots of in vitro grown two weeks old plants cultured under short day and continuous 
light conditions, it was noticed that AtCRK5 and AtCRK1 had the higher levels of expression among all genes, while AtCRK3 and AtCRK6 displayed the lowest expression levels (Figure 12). The length of light illumination displayed undetectable or only slight influence on transcript levels of the AtCRK genes tested. The primers used for this study can be found listed in Supplementary Figure S1.

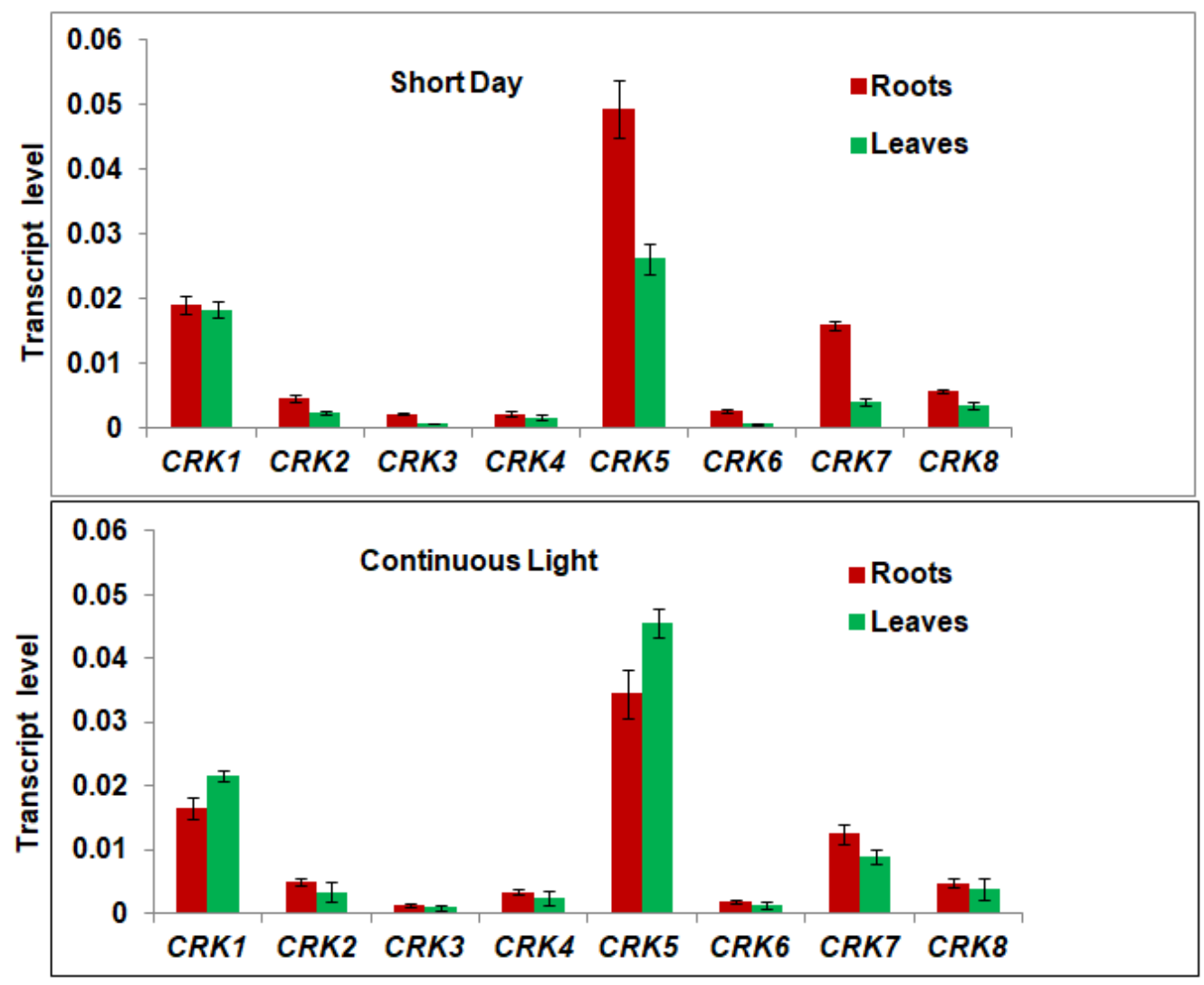

Figure 12: AtCRK gene expression in wild type Arabidopsis plants. Transcript levels were determined in rosette leaves and roots of 14 days old wild-type Arabidopsis plants at short day and continuous light conditions using qRT-PCR. Relative transcipt levels were standardized to GAPDH2. Reactions were made in three replicates. Bars indicate standard deviation (SD) carried out with two independent biological repetitions.

\subsection{Intracellular protein localization of AtCRK protein kinase family}

Various members of the AtCRK protein kinase family from different plant species show highly conserved N-terminal domains with myristoylation/palmitoylation sites (Figure 13). Based on the predicted N-terminal myristoylation sites, AtCRK proteins were assumed to be membrane localized. (Lu and Hrabak, 2013; Rigó et al., 2013). Subcellular protein localization database search (SUBA) for the predicted localization of AtCRK proteins and their annotation can be found in Supplementary Figure S5. 


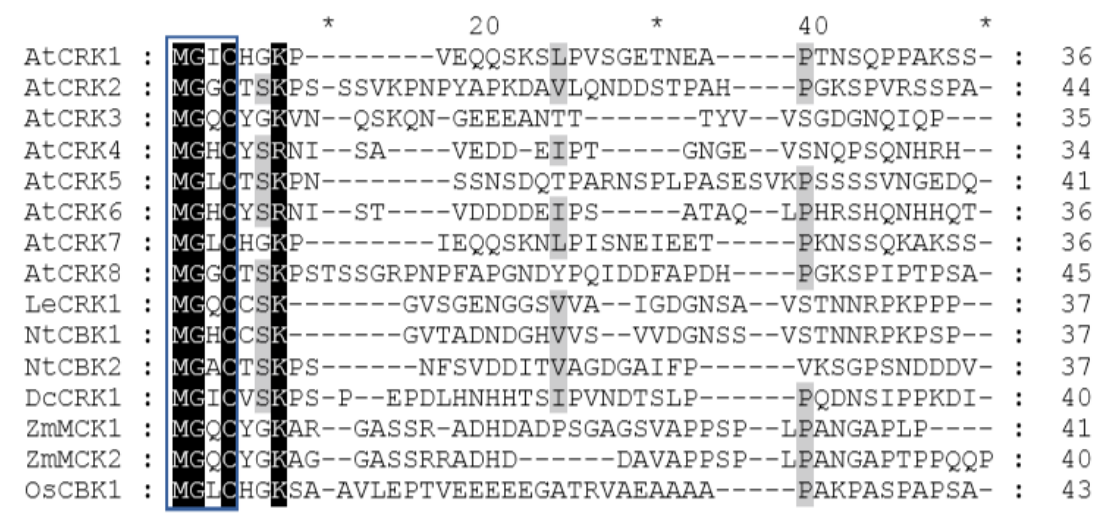

Figure 13: Sequence alignment of $\mathbf{N}$-terminal myristoylation/palmitoylation. Order of sequence alignment of domains of AtCRK family members from different plant species. At: Arabidopsis thaliana, Le: Lycopersicon esculentum, Nt: Nicotiana tabacum, Dc: Daucus carota, Zm: Zea mays, Os: Oryza sativa (Rigó et al., 2013).

\subsubsection{Protoplast transient expression assay}

To verify the cellular distribution, AtCRK protein fusions proteins were transiently expressed under the control of the pCaMV35S promoter in Arabidopsis protoplasts and their subcellular localization was monitored with a confocal laser scanning microscope (CLSM) (Figure 14). As predicted, all transformed cells exhibited GFP fluorescence in plasma membrane (PM) except the AtCRK1 cells which displayed possibly endomembrane GFP fluorescence.

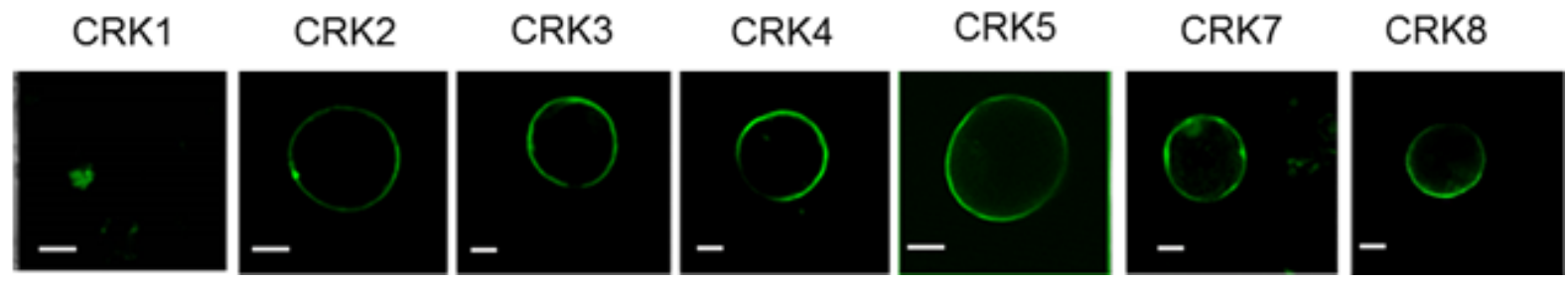

Figure 14: Transient expression of pCaMV35S::CRKs-eGFP in Arabidopsis protoplasts. All but one AtCRK family members displayed plasma membrane (PM) localization in protoplast transient expression assays. The only exception is the $35 \mathrm{~S}:$ :cCRK1-eGFP where the GFP signal is associated probably to endomembranes. (Scale bars $=10 \mu \mathrm{m})$.

\subsubsection{In planta subcellular localization 35S::cCRK-eGFP fusion proteins}

At the plant level the subcellular localization of all AtCRK proteins containing the 35S::cCRK-eGFP fusion in the roots of T3 generation transgenic plants grown in short-day conditions was investigated by confocal laser scanning microscopy (CLSM). We noticed the GFP-derived fluorescence in the meristematic zone of roots, which was associated to 
the perimeters of the cells and on colocalization with a plasma membrane-specific FM4-64 stain indicating plasma membrane localization. The exceptions were the 35S::cCRK1eGFP and 35S::cCRK7-eGFP fusions were the fluorescence was slightly diffused in meristematic cells of roots assuming an additional endomembrane location of these proteins. This endomembrane-like signal in cytosolic region was highly pronounced in case of 35S::cCRK1-eGFP ruling out it to be mostly endomembrane located (Figure 15).
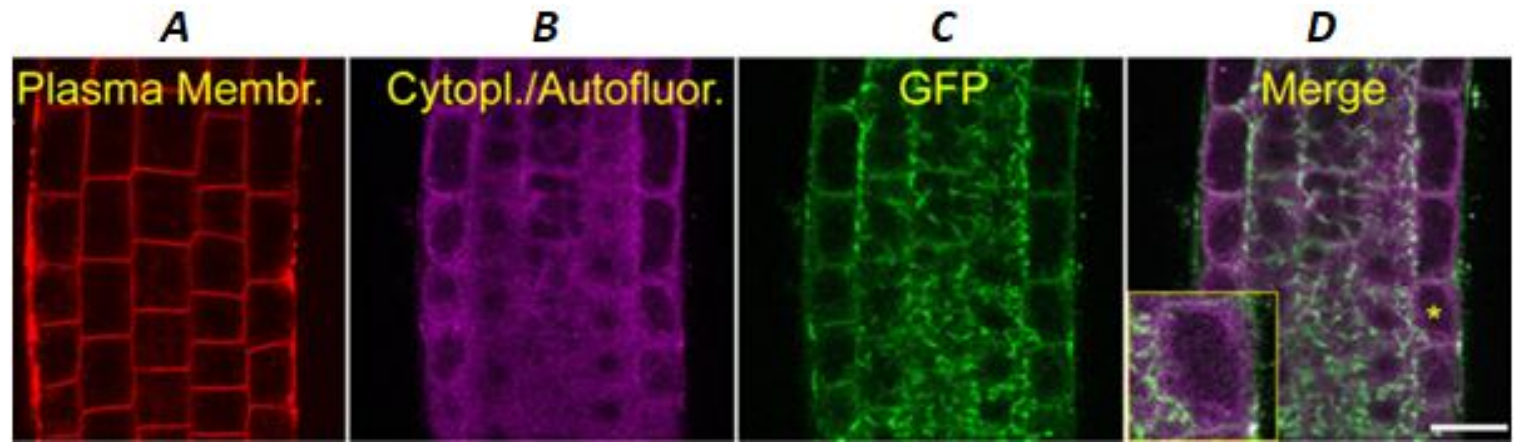

Figure 15: Intracellular localization of AtCRK1-eGFP fusion protein. Intracellular localization of the AtCRK1-eGFP fusion protein. AtCRK1 protein localization in root cells of transgenic Arabidopsis plants expressing the pCaMV35S::CRK1-eGFP gene construct. (A) FM4-64 dye labeling indicates plasma membranes (red, first column). (B) Violet laser induced (excitation: $405 \mathrm{~nm}$ ) cytoplasmic autofluorescence is shown in the second column (pseudocolored in magenta, detected between 425-475 nm). (C) GFPconjugated AtCRK1 image (green) is merged (D) with autofluorescence image at the last column where vacuoles appear as dark intracellular areas due to lack of autofluorescence and absence of AtCRK. Yellow (*) asterisks indicate regions from which $2 \mathrm{x}$ magnified closeup images (insets) are prepared. (Scale bars $=20$ $\mu \mathrm{m})$.

In case of dark grown seedlings of 35S::AtCRK1-eGFP we could also notice the same fluorescence pattern of the fusion protein AtCRK1-eGFP, which displayed a partial PM association and mostly showed a diffused pattern in endomembrane region. We did not notice any prominent differences in the localization pattern of AtCRK1 in short day and dark grown seedlings (Figure 16). 


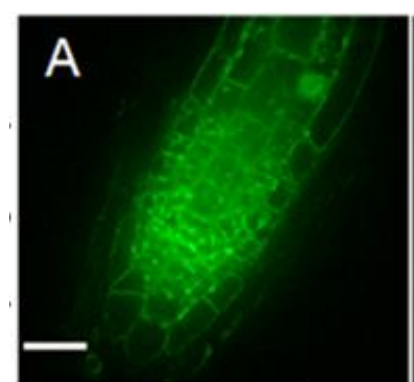

GFP

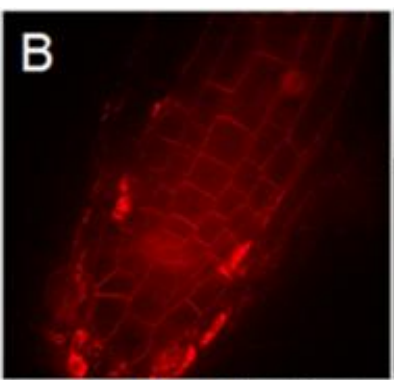

FM-464

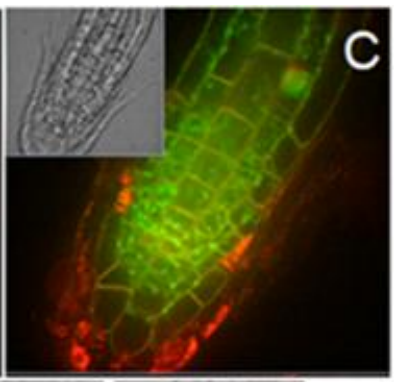

Merge

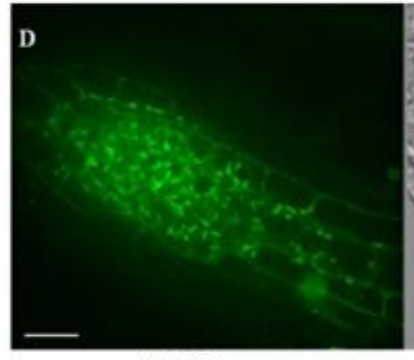

GFP image

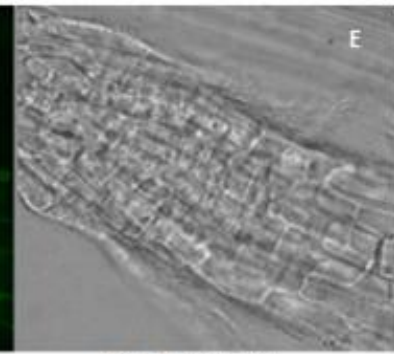

Bright field image

Figure 16: Intracellular localization of the AtCRK1-eGFP fusion protein in dark grown seedlings: AtCRK1 protein localization in root cells of transgenic Arabidopsis plants expressing the pCaMV35S::CRK1-eGFP gene construct. (A) GFP fluorescence (B) FM4-64 dye labelling (C) Merged (D) GFP image and $(\mathrm{E})$ bright field image $($ Bars $=15 \mu \mathrm{m})$.

This intracellular spotted localization pattern of 35S::cCRK1-eGFP protein seems to resemble like mitochondrial or Golgi like localization. But on higher resolution spinning disc confocal microscopy analysis combined with organelle specific markers we ruled out that these structures are not related to mitochondria (Mitotracker Orange labeling, Supplementary Figure S6 or Golgi apparatus (Golgi-specific mCherry expression, Supplementary Figure S7. Online database consisting of protein/dye localization to find a similar localization pattern of a protein or an organelle did not prove to be much beneficial to find the nature of such endomembrane structures. Therefore, the exact nature of these bulbous interconnected membranous structures remained to be elucidated by further labelling with other organelle- specific markers. The plasma membrane localization of the rest of 35S::CRK-eGFP fusion proteins was in accordance with their predicted $\mathrm{N}$-terminal myristoylation. This was confirmed by counterstaining with plasma membrane specific FM4-64 stain which colocalized with the GFP fluorescence and the cytoplasmic autofluorescence was ruled out by violet laser induction (Figure 17-18). 


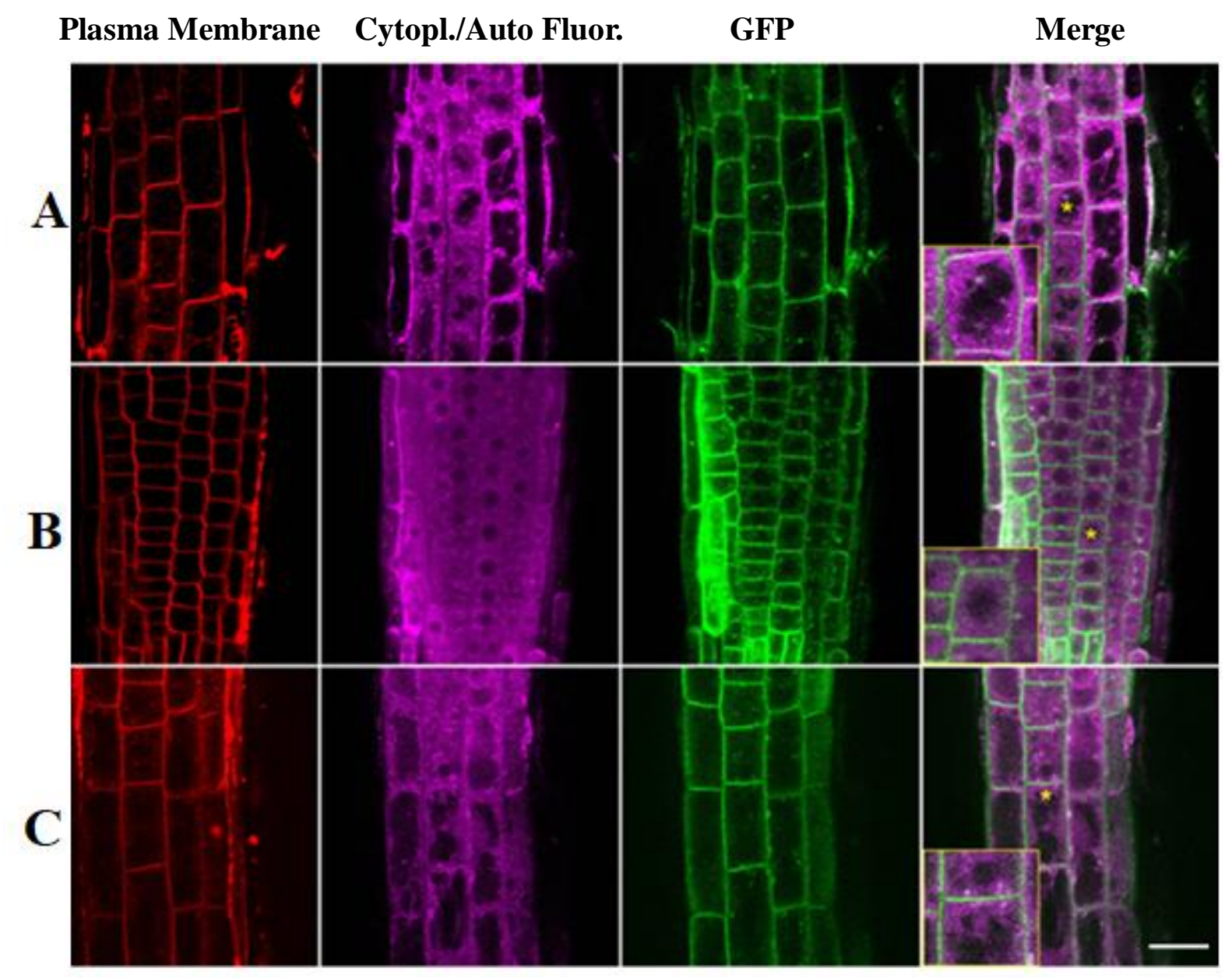

Figure 17: Intracellular localization of the (A) AtCRK2-eGFP, (B) AtCRK3-eGFP and (C) AtCRK4eGFP fusion proteins: AtCRK-eGFP protein localization in root cells of transgenic Arabidopsis plants expressing the pCaMV35S::CRK-eGFP gene construct. FM4-64 dye labeling indicates plasma membranes (red, first column). Violet laser induced (excitation: $405 \mathrm{~nm}$ ) cytoplasmic autofluorescence is shown in the second column (pseudocolored in magenta, detected between 425-475 nm). GFP-conjugated CRK image (green) is merged with autofluorescence image at the last column where vacuoles appear as dark intracellular areas due to lack of autofluorescence and absence of AtCRK. Yellow asterisks indicate regions from which $2 \mathrm{x}$ magnified close-up images (insets) are prepared. (Scale bar $=20 \mu \mathrm{m}$ ). 


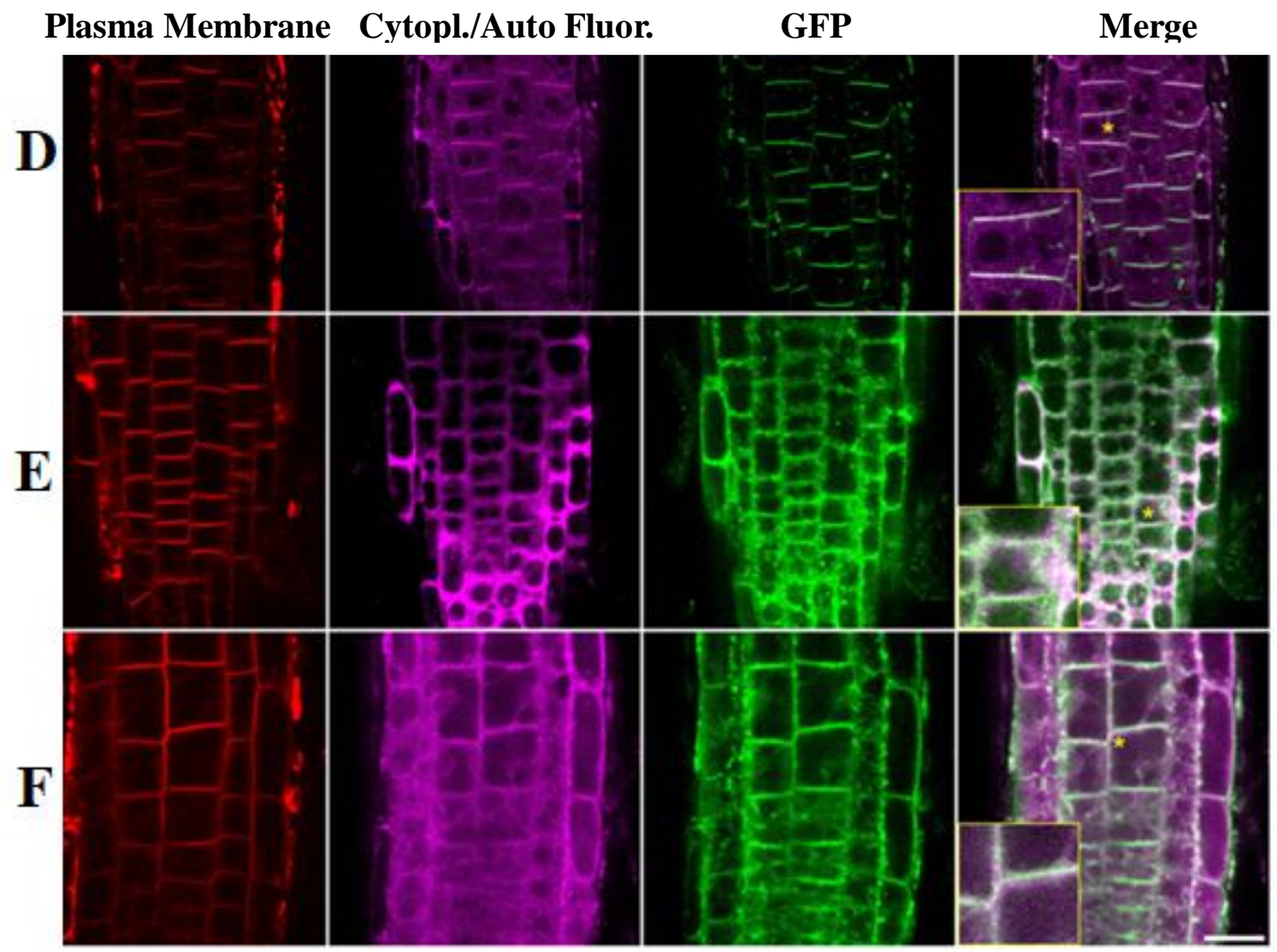

Figure 18: Intracellular localization of the (D) AtCRK5-eGFP, (E) AtCRK7-eGFP and (F) AtCRK8eGFP (E-G) fusion proteins. AtCRK-eGFP protein localization in root cells of transgenic Arabidopsis plants expressing the pCaMV35S::CRK-eGFP gene construct. Same parameters were used as were in Fig. 15 (Scale bar $=20 \mu \mathrm{m}$.).

\subsubsection{Differential centrifugation and western hybridization of AtCRK- eGFPs}

To support the microscopic studies of protein localization, various fractions of 35S::AtCRK-eGFP fusion proteins in cytosolic and microsomal extracts were prepared from transformed Arabidopsis plants by differential centrifugation followed by immunoblotting which revealed, that AtCRK-eGFP proteins were present in the microsomal membrane fractions. This observed microsomal membrane specificity of these AtCRK proteins corelated with the presence of N-terminal myristoylation sites in all AtCRKs which predicts their membrane localization (Rigó et al., 2008; Lu and Hrabak, 2013, Rigó et al., 2013). Though all AtCRKs were detected in microsomal membrane fraction but in case of AtCRK1-eGFP it was detected both in the mitochondria/endomembrane and microsomal membrane fractions (Figure 19). The weak signal intensity of AtCRK2-eGFP and AtCRK3-eGFP in the microsomal fraction is probably the consequence of protein degradation during protein isolation. 


\section{CRK1-GFP}

A

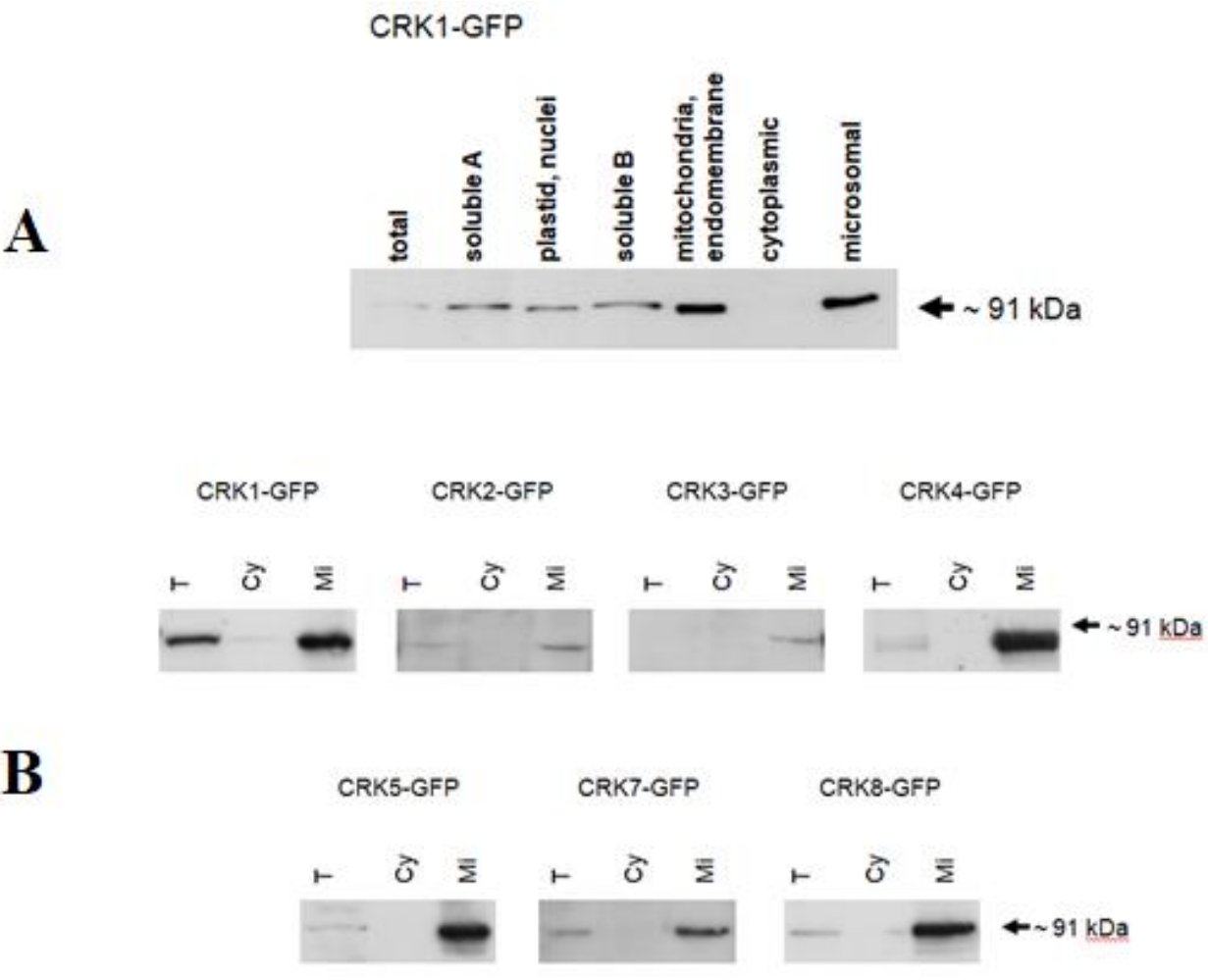

Figure 19: Distribution of AtCRK-eGFP proteins in subcellular fractions. Cellular extracts were separated by differential centrifugation and CRK-eGFP was detected by Western hybridization with an antibody recognizing GFP (anti-GFP). Equal amounts of protein $(25 \mu \mathrm{g})$ were loaded in each lane. Identification of (A) AtCRK1-eGFP in six cellular fractions, separated by differential centrifugation. Distribution of (B) AtCRK1-eGFP, AtCRK2-eGFP, AtCRK3-eGFP, AtCRK4-eGFP, AtCRK7-eGFP and AtCRK8-eGFP in two cellular fractions. All AtCRK-eGFP proteins were detected in the microsomal fractions and but not in the cytoplasmic soluble fractions. T: total protein extract, Cy: cytoplasmic soluble, Mi: microsomal membrane fraction.

\subsection{Functional analysis of the AtCRK genes}

\subsubsection{T-DNA insertion mutants of AtCRK family}

Identification and acquisition of various Atcrk T-DNA insertion mutants was done from public mutant collection lines ordered from Salk Institute, California, USA. Further the genotyping of T-DNA insertion mutants was performed to generate homozygous mutant lines. The position of T-DNA insertion in the genotyped lines can be seen in (Figure 20 ), with the depiction of left border (LB) and right border (RB) of the insert. The arrow head in the direction of left border displays the $35 \mathrm{~S}$ promoter driven expression of the $\mathrm{T}$ DNA insert. The presence of T-DNA insertions in AtCRK6 and AtCRK7 genes could not be confirmed from the various lines and thus were not used in this study. The Atcrkl-1 mutant line was identical which was used in earlier published studies (Liu et al., 2008; Tao and Lu, 2013) and the knockout mutant Atcrk5-1 which was used in this study was the same mutant which was already reported to have a role in root gravitropic responses (Rigó et al., 2013). 
CRK1(At2g41140)

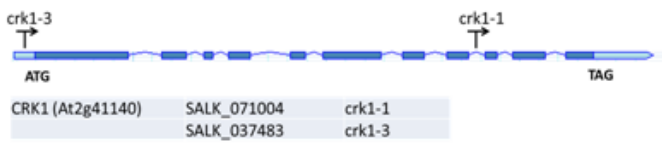

CRK3(At2g46700)

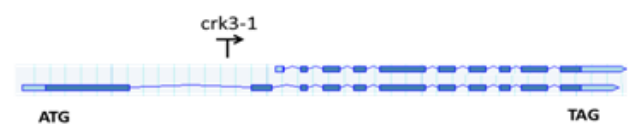

CRK3 (At2g46700) SALK_142932 Crk3-1
CRK2(At3g19100)

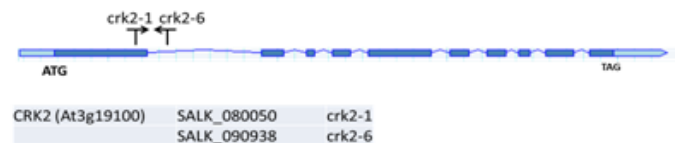

CRK4(At5g24430)

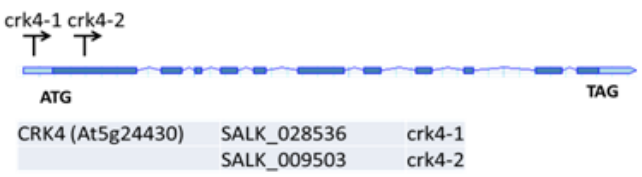

\section{CRK8(At1g49580)}

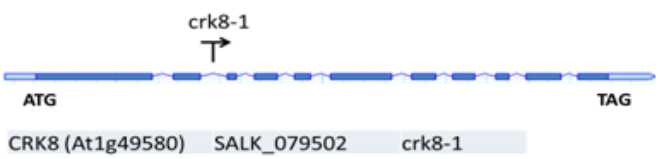

Left Border(LB) 4 Right Border (RB)

Figure 20: Schematic map of the gene with positions of the T-DNA insertions of various Atcrk mutants. Map of the location of T-DNA insertion of the various Atcrk mutants, with arrow heads depicting left border and the end of arrow is right border of the T-DNA insert.

\subsubsection{Expression levels of Atcrk mutants}

After the genotyping, these homozygous mutants were analyzed for knockout or knockdown status by the analysis of their expression studies in mutant plant material (Figure 21). The qRT-PCR analysis of the mutants was performed on the desired lines found to be homozygous for T-DNA insertion and have been further used for phenotyping studies. The primers used for mapping T-DNA insertions and testing transcript levels by qRT-PCR are listed in Supplemental Figure S1.
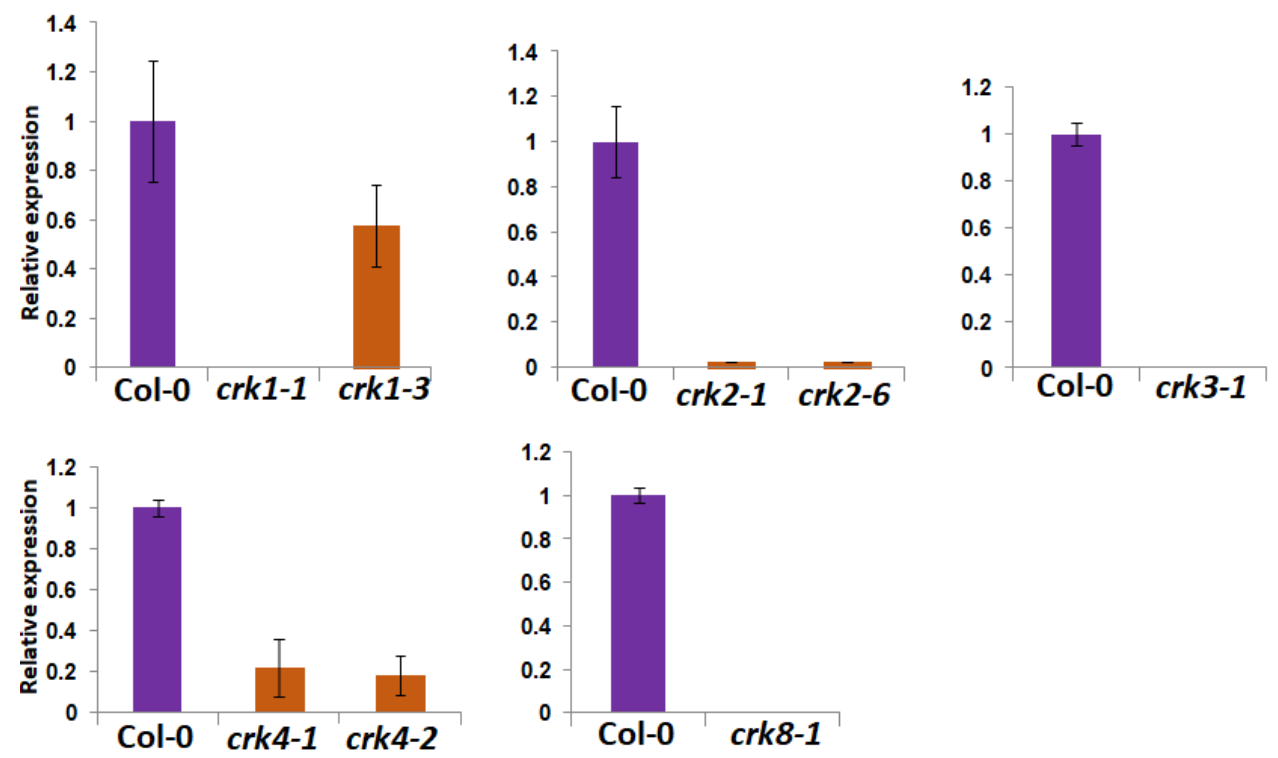

Figure 21: Transcript levels of the Atcrk mutants. Relative expression of various Atcrk mutants used in the study to verify the knockout and knockdown status of these mutants. 


\subsection{Phenotypic characterization of the Atcrk mutants}

Phenotypic characterization of all the above mentioned Atcrk mutants was carried out with a series of assays in which germination and root growth rate responses of the mutants were tested. Germination assay was performed by comparing the counting rates of cotyledon opening of mutant seeds in short day conditions comparing them to wild type Col-0 seeds. Germination in case of all the mutants was found to be delayed with different degrees. It was evident that $80 \%$ of wild type seeds germinated after one day incubation in the culture room, while in the mutants $20 \%$ to $40 \%$ of the seeds germinated by this time. This delay in case of mutant seeds was temporary as nearly afterwards on the third day almost all of mutant seeds germinated (Figure 22). In short-day light conditions when mutant seedlings where allowed to grow on standard culture media, they did not show almost any noticeable growth defects.

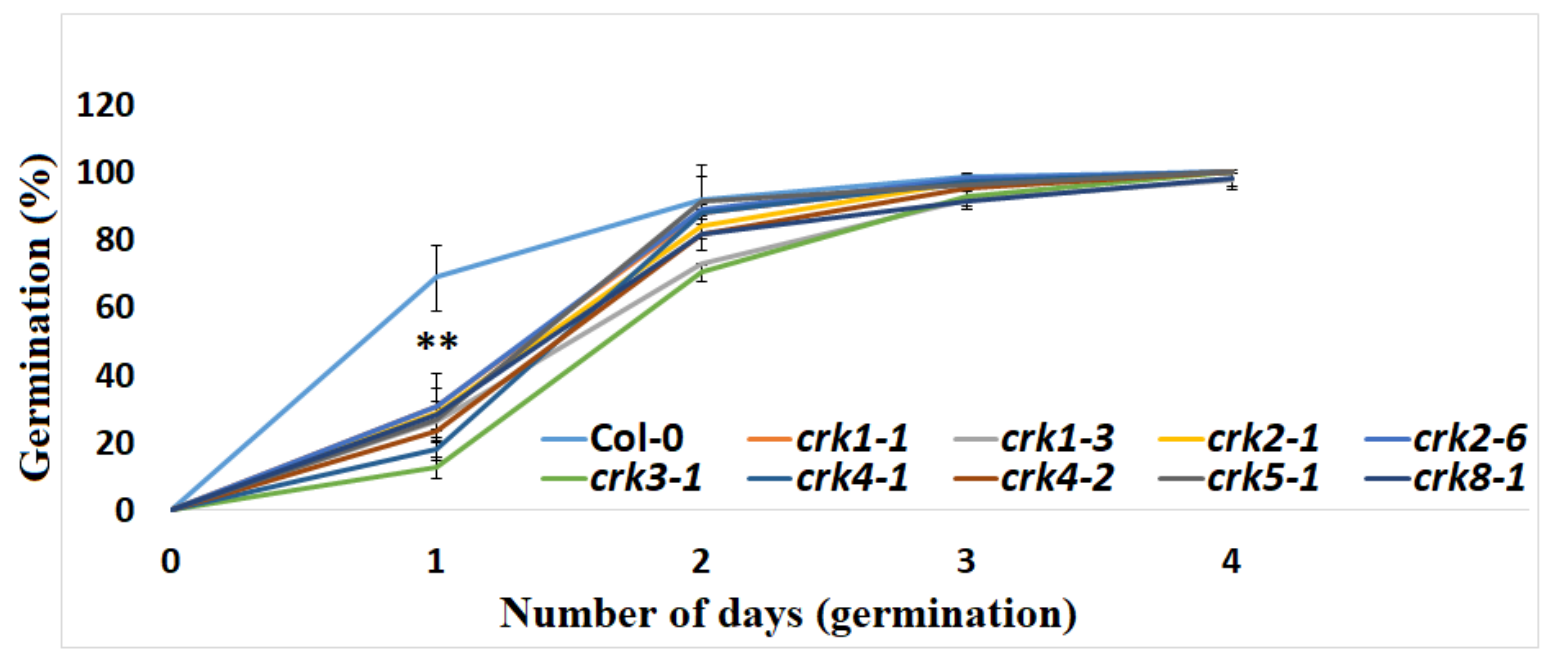

Figure 22: Germination assay of Atcrk mutants. Germination of Atcrk mutants on standard 1/2 MS culture medium in short day conditions. Statistically significant values were calculated by Student's t test $* * \mathrm{p} \leq 0.01$ for crk $1-1$, crk2-1 and $* \mathrm{p} \leq 0.05$, for the rest of Atcrk mutants $(\mathrm{n}=30$, two independent biological repeats). Values are mean \pm SE. 
Earlier it was reported that inactivation of AtCRK5 could inhibit root elongation and delay gravitropic bending of roots (Rigó et al., 2013). Therefore we first tested in all Atcrk mutants the root growth rates in continuous light conditions. On investigation of the the root growth rates by measuring the root length at variable time points for all Atcrk mutants from 5th to 11th days after germination, we could notice that most of the Atcrk mutants displayed reduced root elongation rate in such conditions. This noticeable difference in the root growth rate of Atcrk2-1, Atcrk4-1 and Atcrk5-1 mutants was significantly reported lower in these mutants, while a smaller but not significant reduction in growth rate was noticed in other three mutants of Atcrk1-1, Atcrk1-3 and Atcrk8-1. The only exception in this case was the Atcrk3-1 which did not show any difference in root growth rate (Figure 23).

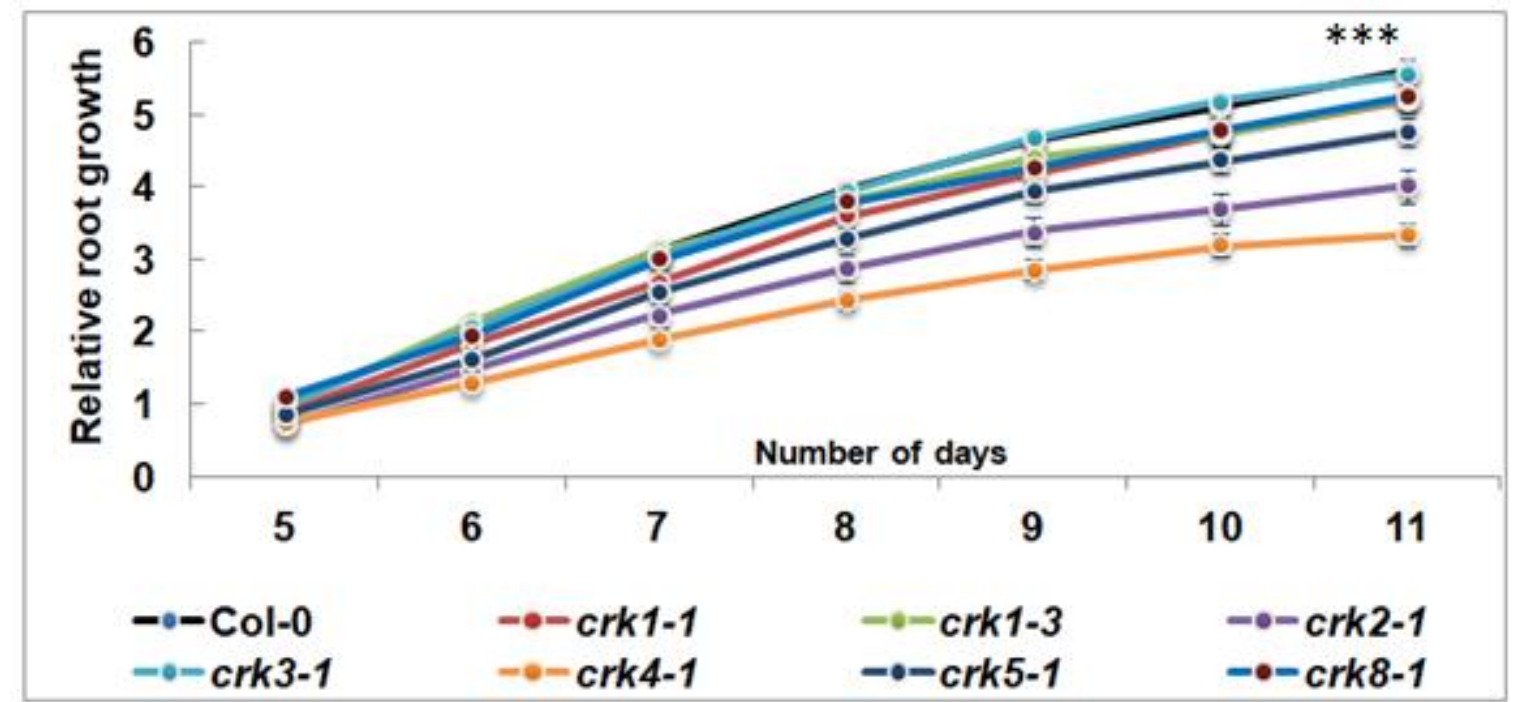

Figure 23: Relative root growth of Atcrk mutants. Relative root growth rate of the mutant seedlings grown on vertical plates for 14 days under continuous light conditions. Statistical p-values represent only the mutants with significant changes for all days of Atcrk2-1, Atcrk4-1 and Atcrk5-1 by Student's t test *** $\mathrm{p} \leq$ 0.001 ( $\mathrm{n}=30$, two independent biological repeats). Values are mean $\pm \mathrm{SE}$.

Further we were interested in the gravitropic response of the Atcrk mutants so both the root and hypocotyl bending assay was performed and their bending capacities were recorded. In case of all the mutants a considerable amount of delay in root gravity response was observed with different degrees of bending when grown under continuous light conditions (Figure 24). 


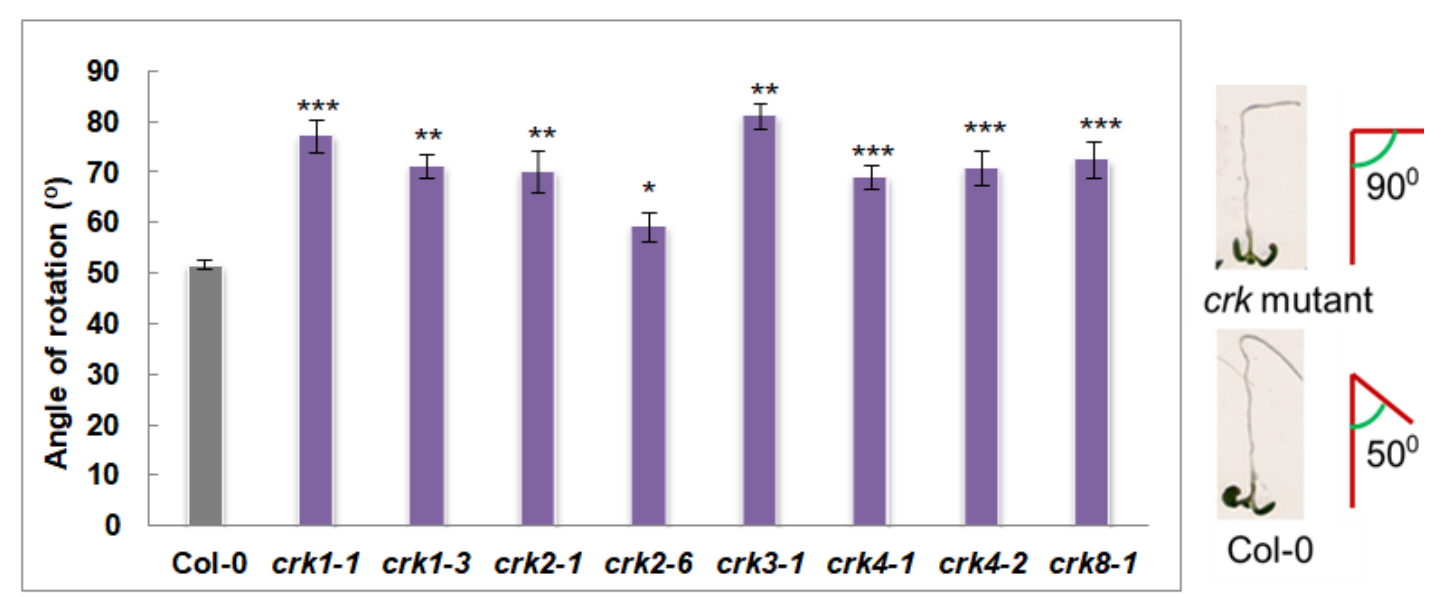

Figure 24: Root gravitropic test. Six days-old, vertically grown seedlings in continuous light were gravistimulated by changing orientation of plates by $135^{\circ}$, and angle of root bending was recorded 24 hours after reorientation. Pictogram depicts the mode of root bending measurements. Values are mean \pm SE. Statistically significant values were calculated by Student's t test for * $\mathrm{p} \leq 0.05, * * \mathrm{p} \leq 0.01$ and *** $\mathrm{p} \leq$ 0.001 ( $\mathrm{n}=30$, two independent biological repeats).

The hypocotyl bending response of the seedlings grown in dark conditions was also found to be delayed in all Atcrk mutants (Figure 25). In analogy with the AtCRK5, this defect of the delayed gravity response in both roots and hypocotyls is suggested to be a consequence of disturbed auxin response and its regulation, which is essential for proper root or hypocotyl bending during gravitropic stimulation (Zádníková et al., 2010; Abbas et al., 2013; Zádníková et al., 2016).

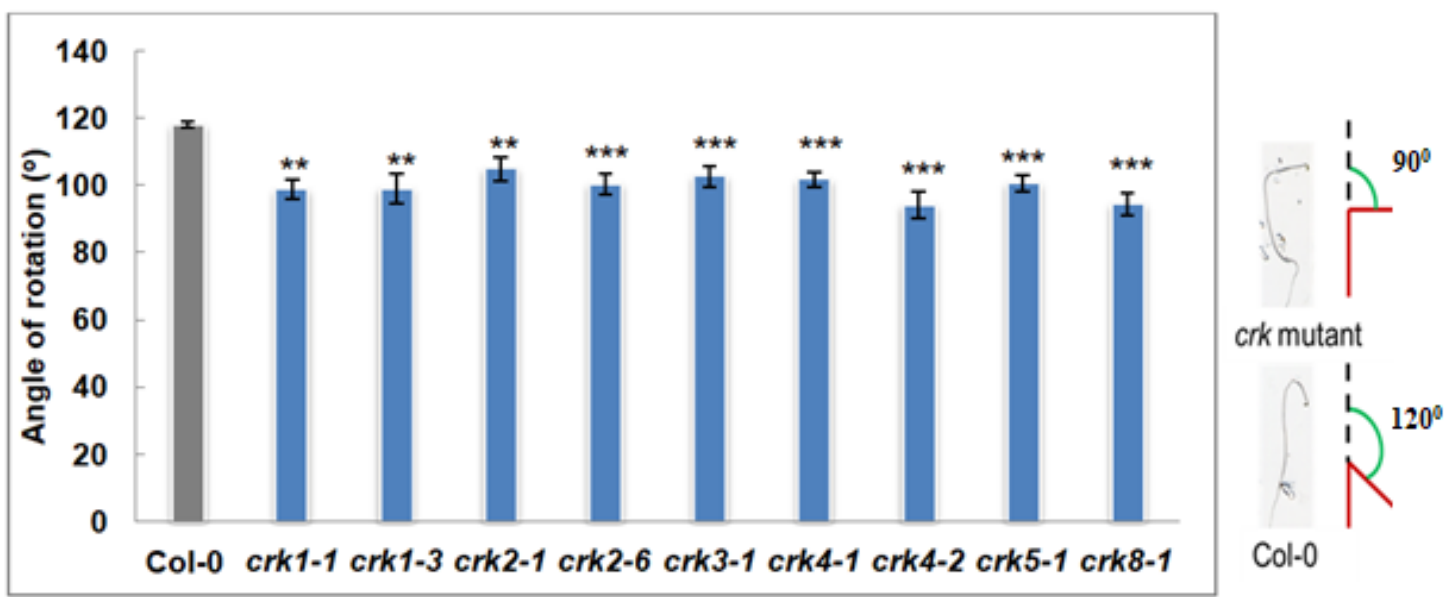

Figure 25: Hypocotyl gravitropic test. Six days old seedlings were germinated in dark, and plates were reoriented 5 days after germination. Hypocotyl angles were measured after 48 hours of reorientation. Pictogram depicts the the mode of hypocotyl bending measurements. Values are means \pm SE. Statistically significant values were calculated by Student's $\mathrm{t}$ test for * $\mathrm{p} \leq 0.05$, ** $\mathrm{p} \leq 0.01$ and *** $\mathrm{p} \leq 0.001$ ( $\mathrm{n}=30$, two independent biological repeats).

Previously in case of one the mutant Atcrk5-1 it was reported to possess an altered localization of PIN2 in roots with a delay in its gravitropic response of roots. The PIN2 auxin efflux transporter is one of the most characteristic player of the basipetal auxin 
transport in Arabidopsis roots (Müller et al., 1998; Zádnikova et al., 2015). Phenotypic characterization of the mutants in the first two described PIN genes revelead that polar auxin transport (PAT) involving the PIN transporters play a very important role in organogenesis and the root gravitropic response (Adamowski and Friml, 2015). Further in vitro kinase studies could formulate the role of this kinase in phosphorylation of the central hydrophilic T-loop of PIN2 which played an important role for its plasma membrane association and its stability (Rigó et al., 2013). Based on the root gravitropic assay we observed that all the other Atcrk mutants displayed a delayed gravitropic bending in roots, therefore the PIN2 immunilocalization was performed on the Atcrk mutant roots to check the distribution of PIN2 transporter in the roots of all mutants.

\subsection{Immunolocalization of PIN2 in roots of Atcrk mutants}

An altered distribution of PIN2 protein was observed in the mutants with improper localization pattern when compared to wild type roots. Distribution of normal PIN2 pattern in epidermal and cortex cells of transition zone in wild type Col-0 roots was seen, but an aberrant PIN2 localization was obseved in transient zone cells of each Atcrk mutant roots (Figure 26). We assume this altered PIN protein distribution to be a consequence of improper phosphorylation of PIN2 by Atcrk mutants which leads to such altered localization pattern in roots, in analogous as was earlier reported in the Atcrk5-1 mutant (Rigó e t al., 2013).

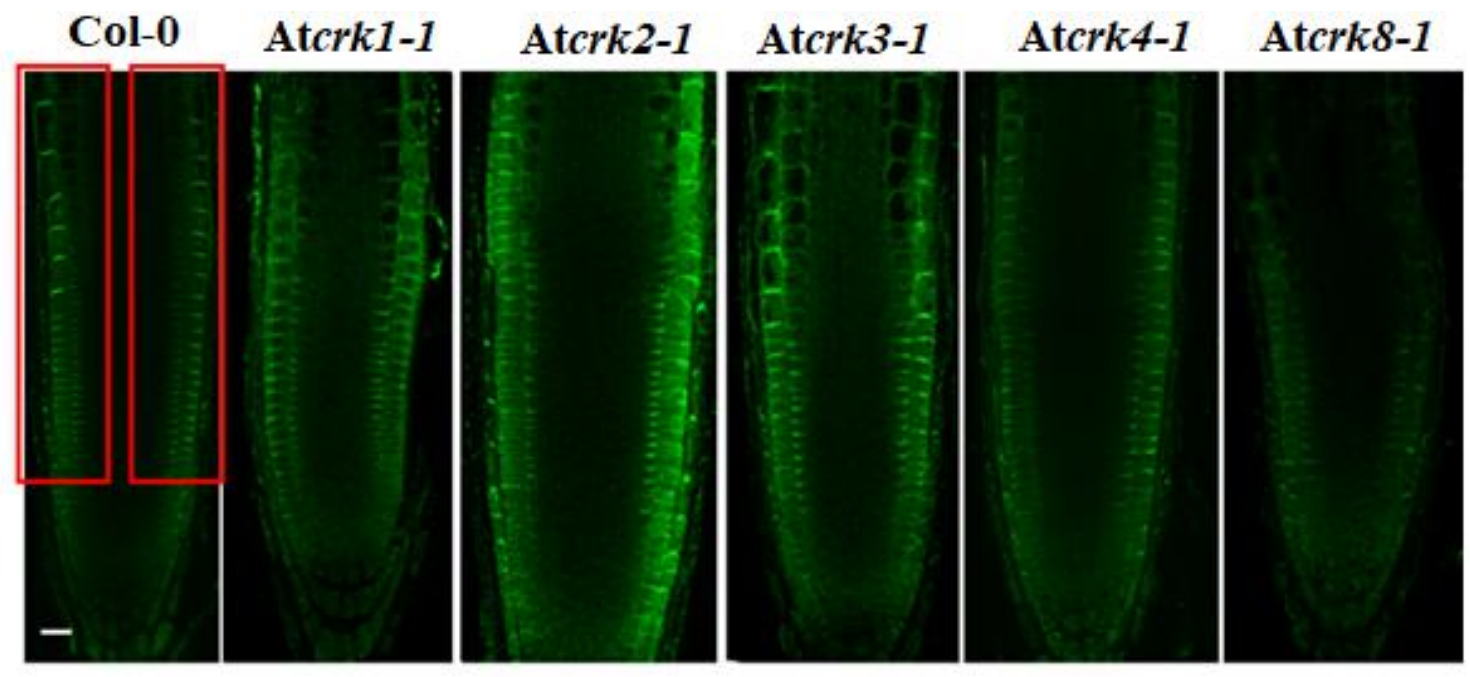

Figure 26: Immunolocalization of PIN2 in wild type Col-0 and the Atcrk mutants. PIN2 (green signal) shows normal localization in epidermal/cortex cells of Col-0 plants. PIN2 signal intensity or pattern is altered in most Atcrk mutants. (Scale bars $=25 \mu \mathrm{m}$ ). Red box in Col-0 depicts the preferable zone of PIN2 localization. 


\subsection{Light sensitive phenotype of Atcrk1-1 T-DNA insertion mutant}

Despite the above phenotypic differences of Atcrk mutants in various light conditions, the short-day illuminating conditions of all the Atcrk mutants did not show much noticeable difference when compared to wild type plants. But in continuous light illumination, one of the mutants of AtCRK family Atcrkl-1 was reported to be sensitive to such long term illuminating growth condition in vitro, displaying a semidwarf phenotype and serious growth defects (Figure 27). In such mutant with continuous illumination, a reduced pattern of rosette size with pale green coloration was observed, with an indication of reduction in chlorophyll content in leaves.

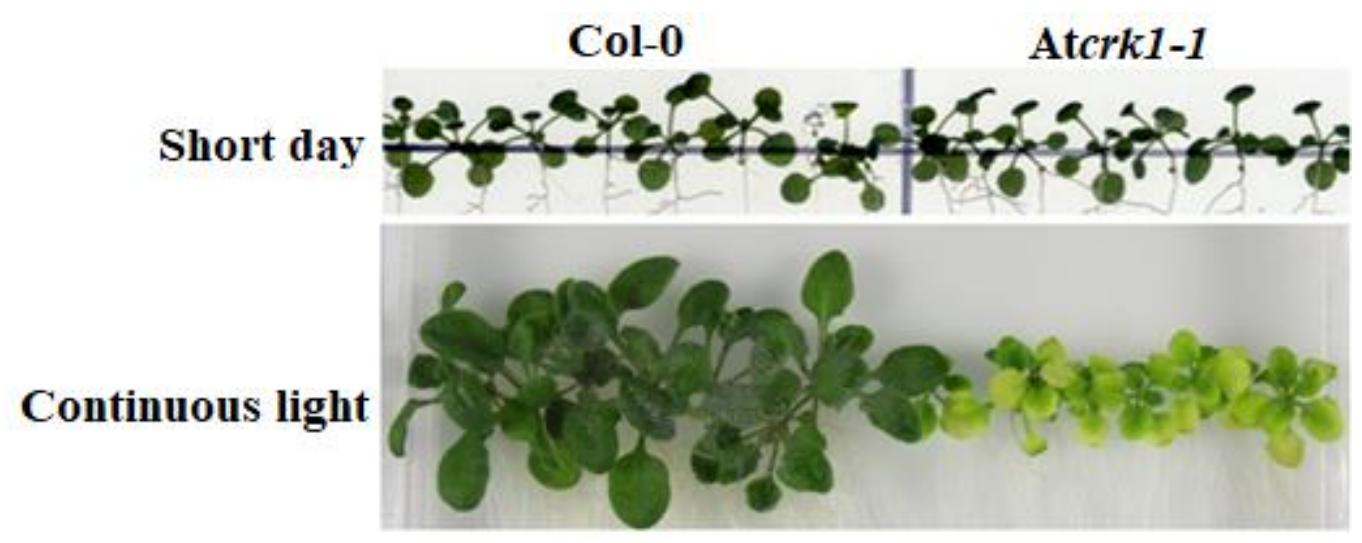

Figure 27: Light sensitivity of the Atcrk1-1 mutant. Images of 3 weeks-old wild type (Col-0) and mutant (Atcrk1-1) plantlets grown on vertical plates under continuous light or short day illumination.

When the fresh weight of such plants was compared, Atcrkl-1 was around more than $60 \%$ less than wild type plants in weight, while in short day condition this difference in the fresh weight did not exceed more than $20 \%$ (Figure 28A). We noticed a considerable amount of decrease in combined chlorophyll $a+b$ content of Atcrk1-1 mutant, which was around nearly $50 \%$ less than that of wild type in continuous light, while this mutant did not show reduced levels of chlorophyll content in short day conditions (Figure 28B). Various pigment contents of the Atcrkl-1 were measured to determine the overall effect on photosynthetic pigment and the decreased values of all the pigments was reported in the mutant. Individual pigments including carotenoids (Figure 29A), chlorophyll $b$ (Figure 29B) and chlorophyll $a$ (Figure 29C) were found to be almost 50\% reduced in the Atcrkl-1 mutant. 

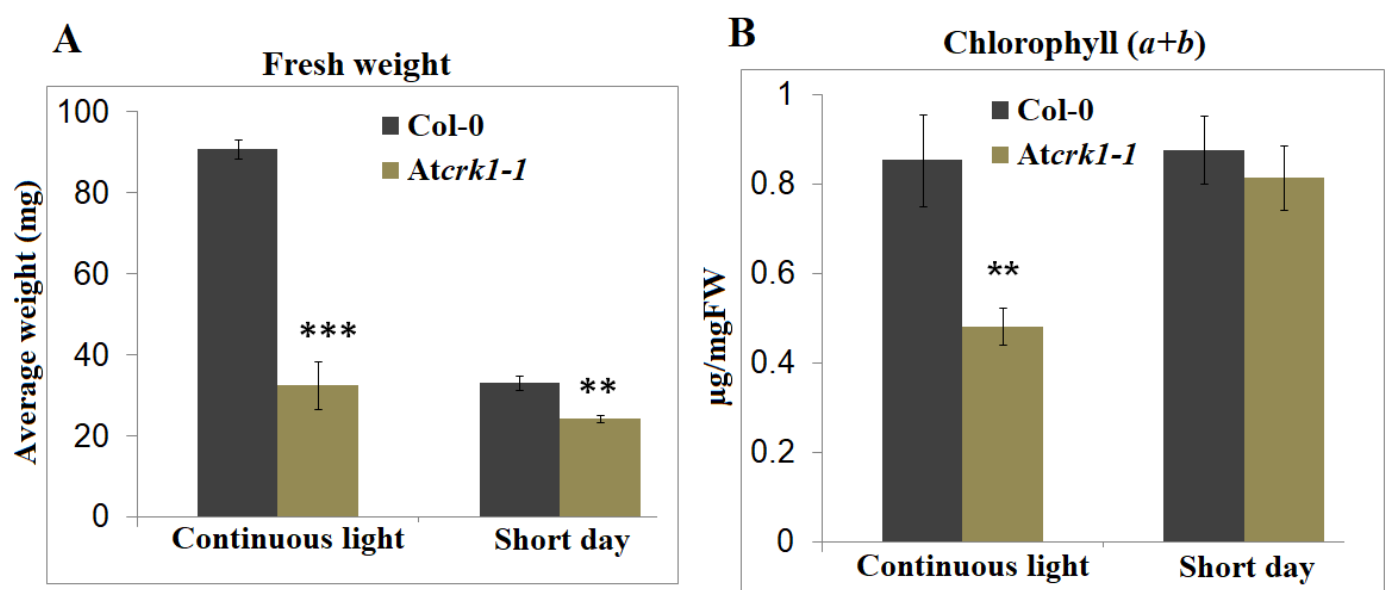

Figure 28: Fresh weight (A) and chlorophyll $a+b$ (B) contents of 3 week-old plantlets under continuous light and short day conditions. Statistically significant values were calculated by Student's $\mathrm{t}$ test for ** $\mathrm{P} \leq$ 0.01 and $* * * \mathrm{P} \leq 0.001$. Values are means $\pm \mathrm{SD}$.

\begin{tabular}{|c|c|c|c|c|c|c|}
\hline $\mathbf{A}$ & & B & & $\mathbf{C}$ & & \\
\hline 0.16 & Carotenoids & 0.4 & Chlorophyll $b$ & & Chlor & yll $a$ \\
\hline 0.14 & & 0.35 & & 0.8 & & \\
\hline 0.12 & & 0.3 & 1 & 0.7 & I & \\
\hline 0.1 & & 0.25 & $* *$ & 0.6 & & \\
\hline 0.08 & ** & 0.2 & $\mathrm{~T}$ & 0.5 & & $\star * *$ \\
\hline 0.06 & & 0.15 & + & $\begin{array}{l}0.4 \\
0.3\end{array}$ & & T \\
\hline 0.04 & & $0.1-$ & & 0.2 & & \\
\hline 0.02 & & 0.05 & & 0.1 & & \\
\hline 0 & Atcrk1-1 & 0 & Atcrk1-1 & 0 & Col-0 & Atcrk1-1 \\
\hline
\end{tabular}

Figure 29: Carotenoid, chlorophyll $\boldsymbol{b}$ and chlorophyll $\boldsymbol{a}$ contents of three weeks-old Atcrk1-1 plantlets. Individual pigment contents of 3 week old Atcrk $1-1$ mutant plantlets grown vertically under continuous light conditions in thermostat room. Values are means $\pm S D, n=15$ seedlings. Statistically significant values were calculated by Student's $\mathrm{t}$ test for $(* * \mathrm{P} \leq 0.01)$.

Further the measurement of photosynthetic efficiency of Atcrkl-1 mutant and wild type plants based on PSII chlorophyll fluorescence was performed as it is one of the compatible methods to determine photosynthetic integrity and capability. It was reported that the reduction in the chlorophyll content can influence the photosynthetic capability of plants. The $F_{\mathrm{v}} / F_{\mathrm{m}}$ parameters of the Atcrkl-1 mutant were found to be reduced when compared to Col-0 wild type plants (Figure 30A). This decreased $F_{\mathrm{v}} / F_{\mathrm{m}}$ values might be the consequence of lower chlorophyll content of Atcrk1-1 mutant (Oh et al., 1996). Under various increasing photosynthetic active radiation (PAR), the reduction in the effective PSII quantum yield $\Phi_{\text {PSIl }}$ values were detected in Atcrk1-1 under all light intensities (Figure 30A). When the electron transport rate (ETR) of the Atcrk1-1 was compared with that of the ETR values of Col-0 wild type plants we could observe that in the case of mutant it was lagging behind when compared with that of the wild type plants, mostly 
reported at higher PAR (Figure 30B), which indicated that adaptation capacity of mutant to higher light intensities is weaker. Indeed the values of regulated non-photochemical quenching (NPQ) in Atcrk1-1 mutant was reported to be lower than that of wild type plants, while the non-regulated non-photochemical quenching (Y (NO)) (Klughammer and Schreiber, 2018) was found higher in mutant plants (Figure 30C,D).

Based on the results of reduced pigment contents and defective PSII capability we conclude that the mutation of Atcrkl-1 results in serious consequences on light harvesting capability and the electron flow. Consequently, the efficiency of photosynthesis and excitation energy quenching ability is impaired in Atcrk1-1 mutant. In the case of other Atcrk mutants we could not observe any such prominent alterations in the photosynthetic parameters under such light conditions of continuous illumination (Supplemental Figure S8).

A

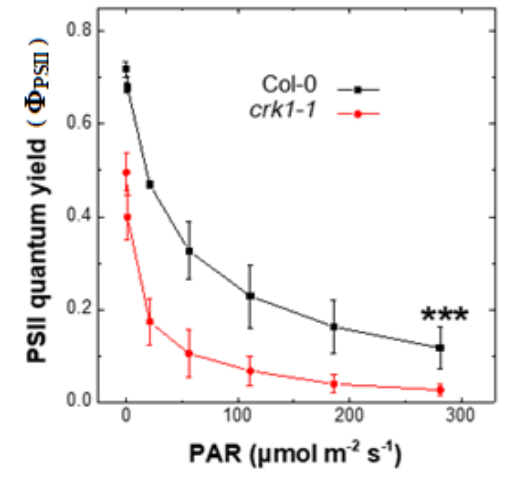

C

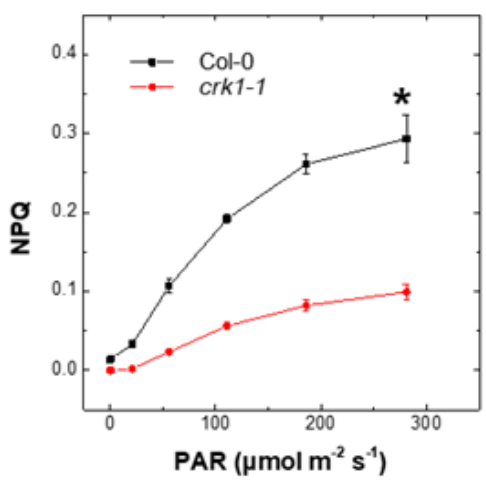

B

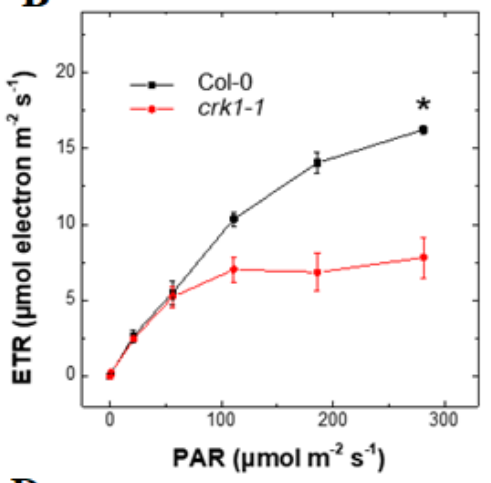

D

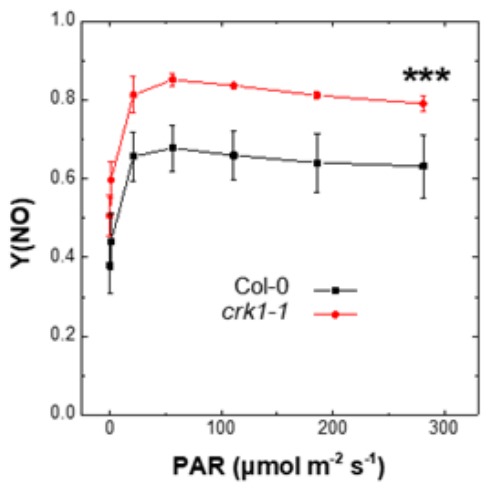

Figure 30: Photosynthetic parameters of Atcrk1-1 mutant under continuous light. (A) Effective PSII maximum quantum yield $\left(\Phi_{\text {PSII }}\right)$. The Atcrkl-1 mutant showed significantly lower effective PSII quantum yield values under all light intensities (***: $\mathrm{p} \leq 0.001)$. (B) Electron transport rate (ETR). The Atcrk1- 1 mutant has significantly lower ETR values than wild type plants $(*: \mathrm{p} \leq 0.05)$. (C) Regulated Non Photochemical Quenching (NPQ). (D) Non-regulated non-photochemical quenching (Y (NO)). The NPQ values of Atcrk1-1 plants were decreased (*: $\mathrm{p} \leq 0.05)$, while the $\mathrm{Y}(\mathrm{NO})$ showed increased $(* * *: \mathrm{p} \leq 0.001)$ values in Atcrkl-1 mutant as compared to wild type plants. Data are presented as the mean \pm standard error. Two-way ANOVA test were used for statistical analysis. 


\subsection{Singlet oxygen $\left({ }^{1} \mathrm{O}_{2}\right)$ accumulation and cell death in Atcrk1-1 mutant}

The decreased photosynthetic efficiency of Atcrk1-1 mutant can be correlated with excess presence of reactive oxygen species (ROS). The ROS are the by-products of photosynthetic processes, and their influence is highly affected during various stress conditions, leading to decreased photosynthetic performance in plants (Apel and Hirt, 2004; Asada, 2006; Li et al., 2009). In the excess light conditions, the most dominant ROS species produced in chloroplast is reported to be singlet oxygen $\left({ }^{1} \mathrm{O}_{2}\right)$, a very important ROS which is quite damaging to plants in excess production and can lead to ROS- induced cell death (Krieger, 2005; Triantaphylidès et al., 2008; Ramel et al., 2013). Production of singlet oxygen is typically associated with less efficient dissipation of photosynthetic energy or it can arise from light reactions by the accumulation of chlorophyll precursors. Such photodynamic singlet oxygen production is believed to be involved in stress signaling and programmed cell death (Mor et al., 2014). The light sensitive phenotype of Atcrkl-1 in continuous illumination instigated us to measure the level of ROS and in particular the singlet oxygen levels in the mutant. The detection of ${ }^{1} \mathrm{O}_{2}$ levels was estimated by fluorescence of the ${ }^{1} \mathrm{O}_{2}$-specific Singlet Oxygen Sensor Green (SOSG) fluorescent probe used in wild type and Atcrk1-1 mutant after treating the seedlings with SOSG probe and illuminated with high light $\left(2300 \mu\right.$ mole photons $\left.\mathrm{m}^{-2} \mathrm{~s}^{-1}\right)$ for 5 minutes (Flors et al., 2006; Hideg, 2008; Rehman et al., 2016 ). The substantial difference in the SOSG fluorescence was observed in ${ }^{1} \mathrm{O}_{2}$ production between the Atcrk1-1 mutant and wild type Col-0 leaves (Figure 31).

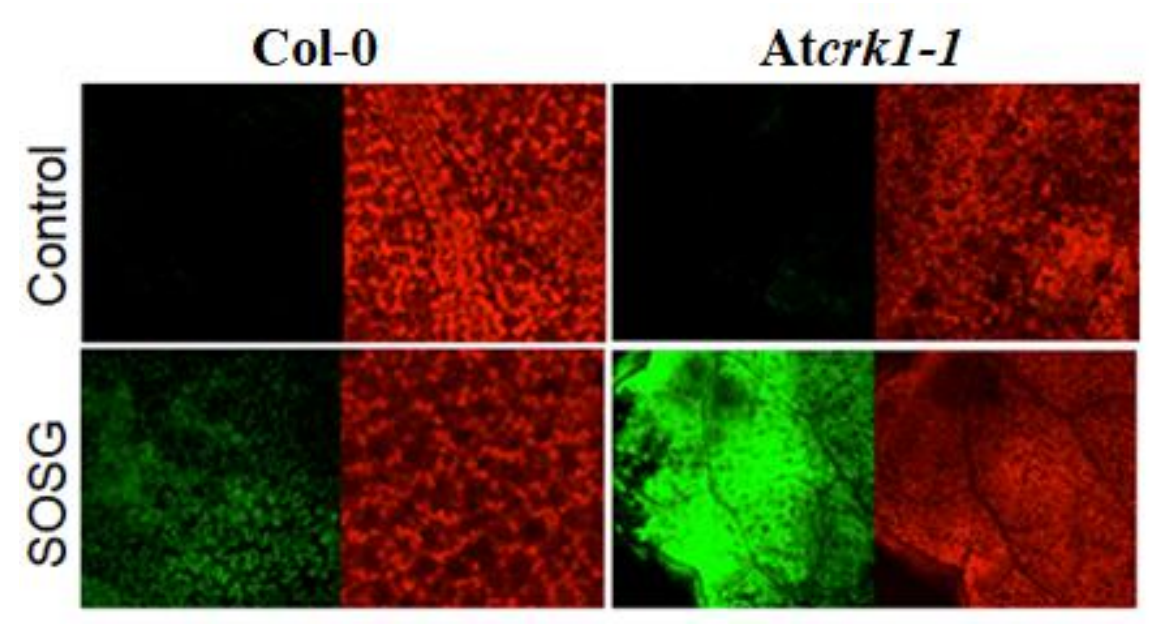

Figure 31: ROS accumulation in Atcrk1-1 mutant. Production of ${ }^{1} \mathrm{O}_{2}$ in leaves of Atcrk1-1 mutant detected by fluorescence of the SOSG probe. Plants were infiltrated with SOSG and exposed to high light (10 $\mu \mathrm{M}$ SOSG, $2300 \mu$ mole $\mathrm{m}^{-2} \mathrm{~s}^{-1}$ for 5 minutes). Fluorescence was detected by CLSM in continuous lightgrown plants. Note the intensive fluorescence of SOSG-infiltrated mutant leaves. 
Since we observed the hyperaccumulation of ${ }^{1} \mathrm{O}_{2}$ in mutant leaves under continuous illumination, we analyzed the implication of this accumulation in the process of ${ }^{1} \mathrm{O}_{2}$ mediated cell death. Thus, its effect on the seedlings was estimated by testing the cell death in leaves of Atcrk1-1 mutant by Evans blue staining comparing it to the wild type. As the singlet oxygen destroys the cell membranes thus the penetration of the Evans stain is higher in case of mutant because of the increased permeability of the cell. This retention of the dye in case of wild type Col-0 leaves was much less than Atcrk1-1 mutant in continuous light but not under short-day illumination, which indicates an extensive cell death in Atcrk1-1 leaves (Figure 32). This difference in the retention of Evans blue stain confirmed that AtCRK1 may influence the light-induced programmed cell death in Arabidopsis thaliana.

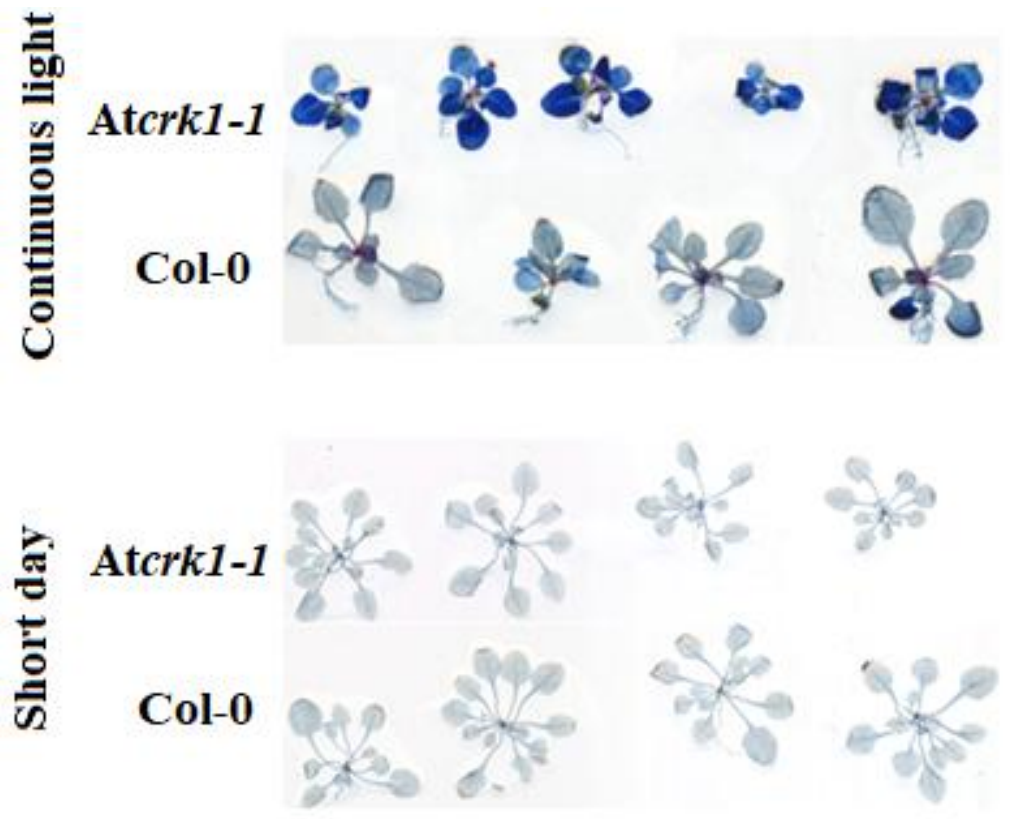

Figure 32: Cell death in Atcrk1-1 mutant. Cell death in leaves of Atcrk1-1 mutant, detected by Evans blue staining. Three weeks-old plants were grown either on short day or under continuous light. Note increased and intense retention of stain (blue reaction) in Atcrk1-1 mutants exposed to continuous light. 


\subsection{Role of AtCRK5 protein kinase in embryogenesis of Arabidopsis}

thaliana

\subsubsection{Decreased seed size and delayed embryo bending phenotype in Atcrk5-1}

As reported in the earlier studies the AtCRK5 protein kinase was reported to be active in most Arabidopsis organs displaying the expression pattern in various parts of seedlings (Rigó et al., 2013), depicting it might be important in other developmental processes. Thus on the basis of our initial observation of the Atcrk5-1 mutant seeds, we found a considerable difference between the wild type Arabidopsis thaliana (Col-0) and the Atcrk5-1 mutant dry seeds when the seed size (perimeter) of these dried mutant seeds was measured, a significant difference in the size of mutant seeds could be noticed (Figure $33 \mathrm{~A})$. After imbibition of these dried seeds at $4{ }^{\circ} \mathrm{C}$, we also measured the average sizes of the length of axis for bent cotyledon from Shoot Apical Meristem (SAM) to Root Apical Meristem (RAM) and a significant difference in the length of the axis in Atcrk5-1 seeds could also be found as compared to wild type Col-0 seeds (Figure 33B). Additionally, on the measurement of the bending angle for the hypocotyl hooks of the bent cotyledon embryos, we noticed that the hypocotyl hook of the Atcrk5-1 mutant embryos were already more opened at this stage as compared to wild type embryos (Figure 33C). 

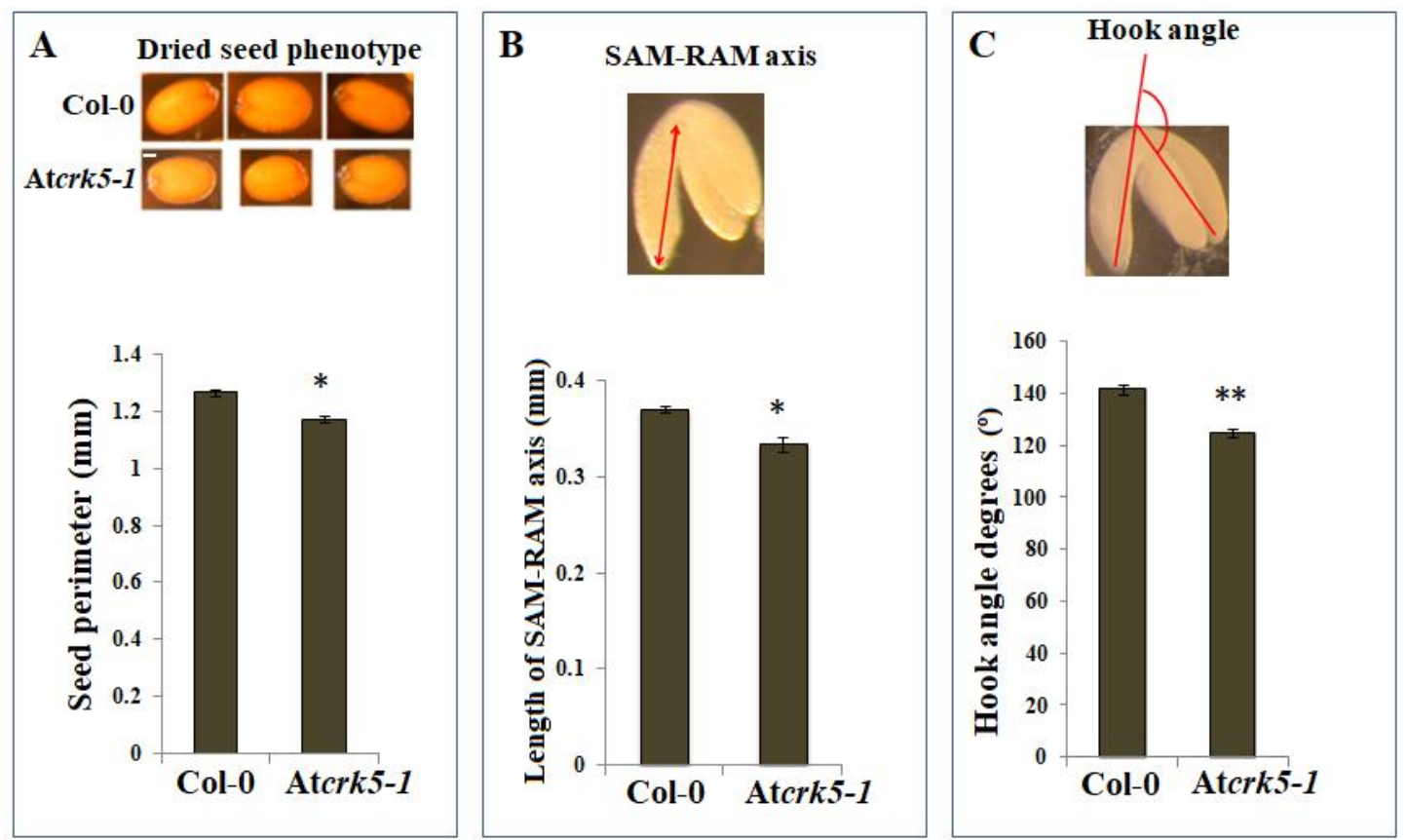

Figure 33: Seed and embryo phenotype of wild type (Col-0) and mutant (Atcrk5-1). (A) Comparison of dry seed size (perimeter) of the wild type (Col-0) and mutant (Atcrk5-1) and quantification of the seed size (perimeter) in wild type and mutant lines after 2 days imbibition at $4{ }^{\circ} \mathrm{C}$. (B) Length of the axis from SAM until RAM is calculated in bent cotyledon stage embryos arised from the (A) experiment, pictogram is a representation for the measurement of SAM-RAM axis. All values are averages of axis of at least 100 bent cotyledon embryos. Standard errors (SE) are also shown. Asterisk $(*)$ depicts significant differences between the wild type and mutant embryos (Student's t-test: ${ }^{*} \mathrm{p} \leq 0.05, \mathrm{n}=100$ ). (C) Decreased hook angle of the embryos of mutant seeds after 2 days imbibition at 4 C. Quantification of the hook angle of wild type and mutant embryos arising from (A) experiment and pictogram is a representation for the measuring embryo hook angle in the embryos in intact seeds. The averages and standard error (SE) are shown. (Student's t-test: $* * \mathrm{p} \leq 0.01, \mathrm{n}=75)$. All experiments were repeated thrice with similar results (Scale bar $=100 \mu \mathrm{m})$.

\subsubsection{Delayed embryo development and morphological changes in the late}

\section{Atcrk5-1 mutant embryos}

After the analysis of seed phenotype and finding the noticeable differences in the mutant seeds, we were interested in the individual sequential developmental stages of the embryos to check the possible morphological differences in the wild type and mutant embryos. Therefore various stages of embryos in case of Col-0 and Atcrk5-1 mutant were analysed by the CELL-R Leica Microscope. The results are presented in the Figure 34. So for the visualization of the intact embryos and their shapes the chloral hydrate treatment was performed on seeds obtained from silique 1 (S1) to silique 11 (S11) from the inflorescence stem of greenhouse plants of same age wild type and mutant plant, where the $\mathrm{S} 1$ represents the youngest and the S11 indicates the oldest siliques after successfull pollination. We could report a delayed sequential shift in Atcrk5-1 embryo stage development initiated from globular/early heart embryo stages found in silique 4 (S4) when compared to wild type embryos (Figure 34). The red arrow in the figure depicts the 
direction of the shift (S5) in stages of mutant embryos and the arrow head directing the sequence shift. These images clearly show that there is a developmental delay noticeable from the globular stage in the Atcrk5-1 mutant embryos during embryogenesis.

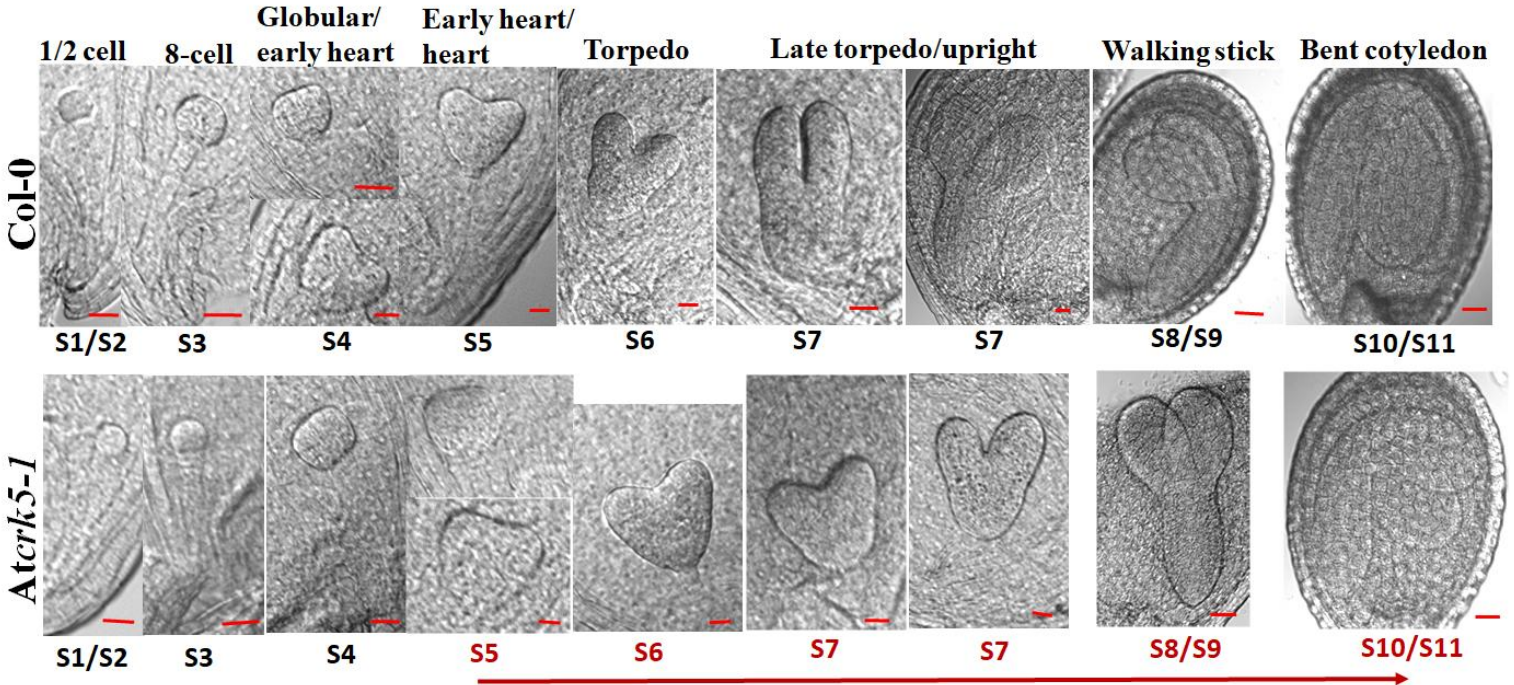

Figure 34: Embryo developmental stages in Atcrk5-1 mutant compared with wild type Col-0. Embryo shapes were visualized after chloral hydrate treatment. A shift in Atcrk5-1 embryo development initiated from globular/early heart embryo stages found in silique5 (S5) as compared to wild type embryos. S1 represents the youngest and S11 the oldest siliques formed after pollination. Note that the wild type (Col-0) bent cotyledon stage embryos have $180^{\circ}$ hook bending, while the same developmental stage mutants (Atcrk51) have much less (approximately around $130^{\circ}$ ) hook bending ability. Red arrow represents the sequential direction of delay (Scale bars $=10 \mu \mathrm{m}$ for S1-S7 and $100 \mu \mathrm{m}$ for S8/S9-S11).

On further quantification of the embryo development (Figure 35), where the quantification of the sequential embryo developmental stages is indicated in percentage in the wild type and Atcrk5-1 siliques by depiction of the different embryo stages by colour coding. This quantification of data indicate that in the wild type siliques the S4/S5 contain basically globular, early heart and heart embryos, while in the same mutant siliques mainly the 8-cell, globular, early heart embryos did appear. The wild type S6 consists of early torpedo and mostly torpedo embryo stages and the mutant S6 contains dominantly heart type and other early embryos. The S7 of wild type siliques have late torpedo/upright embryo stages, while the mutant S7 still have mainly globular, heart and few percentage of early torpedo embryo stages. In the S8/S9 of wild type inflorescence there are already torpedo, upright and walking stick stage embryos with hypocotyl hook bending angle of around $90^{\circ}$, in contrast to the mutant siliques S8/S9 where still the early heart, heart, early torpedo and torpedo with a few percentage of upright embryos are in majority. The wild type S10/S11 already contain principally walking stick and fully bent cotyledon $\left(180^{\circ}\right)$ stage embryos and the same S10/S11 from the mutant contains upright and walking stick embryos with hypocotyl hook bending angle of around $130^{\circ}$. This observation revealed the 
delay in the Atcrk5-1 mutant embryo development by at least two phases compared to the wild type Col-0 embryo development with an already open hypocotyl hook phenotype too.

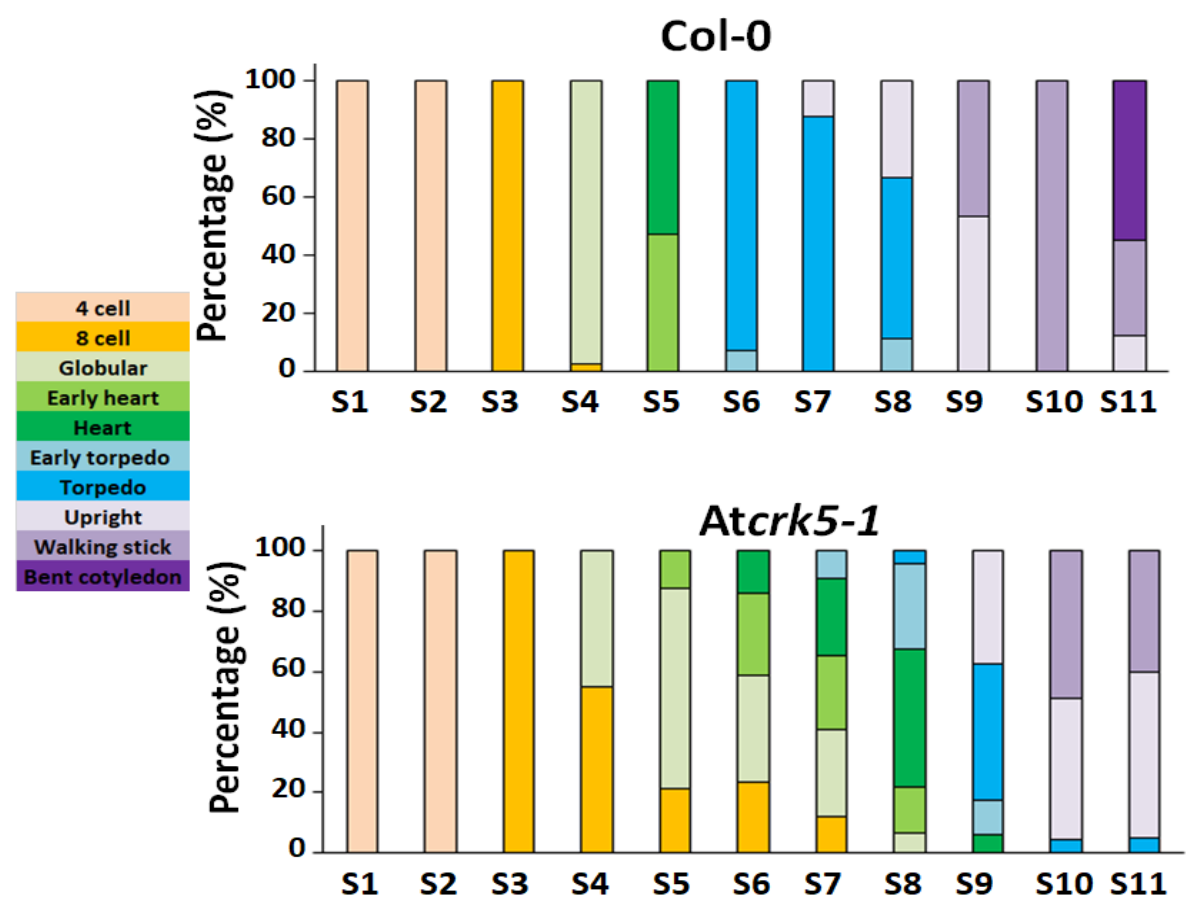

Figure 35: Quantitative analysis of embryogenesis in wild type Arabidopsis thaliana (Col-0) and Atcrk51 mutant correlated to siliques development. Distributions of the different embryo developmental stages from S1 untill S11 depicted in percentage (\%). A notable shift in Atcrk5-1 embryo development from globular embryo stages is noticed when compared to wild type embryos from silique 4 (S4). Minimum 50 seeds were investigated for each genotype for each embryo developmental stages from green house grown plants. The experiment was repeated twice with similar results.

In addition to this developmental stage delay and open hypocotyl hook phenotype in mutant embryos, we could also report that the silique S10-S11 embryos from the Atcrk5-1 mutant were smaller in size when we compared the SAM-RAM ratio of wild type Col-0 embryos to those of mutant embryos localized within the seeds. A significant decrease in mutant embryo axis length was found as compared to length of wildtype axis (Figure 36). 


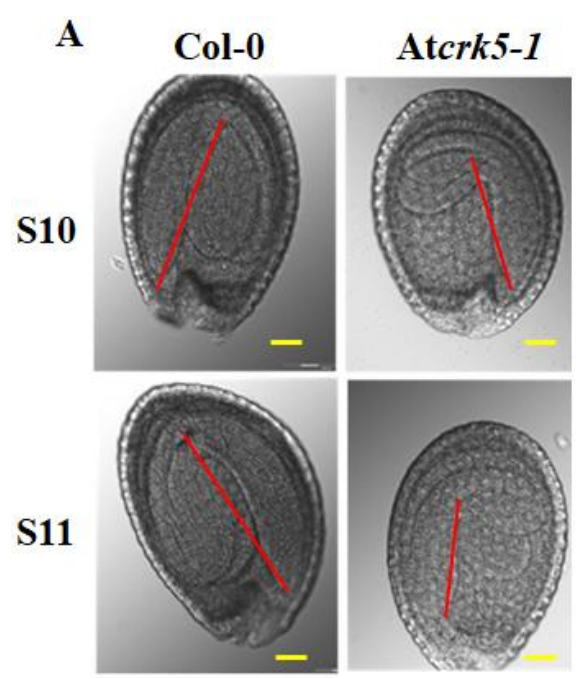

B

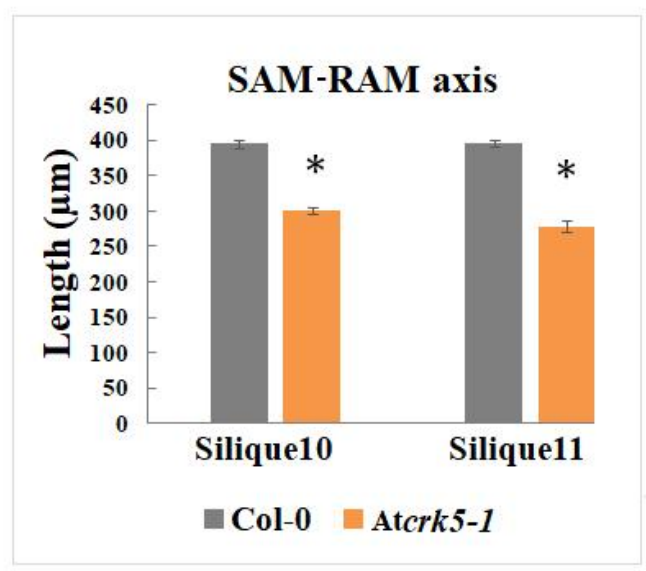

Figure 36: Comparison of SAM-RAM axis size in bent cotyledon embryos within the siliques-derived seeds after precleared in Hoyer's solution. (A) Embryo axis measured from SAM to RAM in silique 10 and Silique 11. Atcrk5-1 embryo axis is shorter in comparision with wild type Col-0 embryo axis. Red line is the depiction of the measurement of axis. (B) Quantification of embryo axis size differences. Asterisk (*) depicts significant differences between the wild type and mutant embryos (Student's t-test: ${ }^{*} p \leq 0.05, n=50$ ); $($ Scale bars $=100 \mu \mathrm{m})$.

\subsubsection{Role of gibberellic acid and auxin cross talk in the development of embryos in Atcrk5-1 mutant}

On noticing such shift in the development of the embryos of Atcrk5-1 mutant during the silique stages S5-S11, we supposed that this shift to be the consequence of shortage of endogenous gibberellic acid (GA) supply of these embryos. This sequential delayed shift in Atcrk5-1 embryo development was visible and started from globular/early heart embryo stages located in silique 5 (S5) when compared with the wild type embryos. The rapid expansion of wild type embryos takes place in this period (from S5 till S11); (Hu et al., 2018). This phenomenon is comparable with increased expression patterns of the bioactive gibberellins involving $\mathrm{GA}_{1}$ and $\mathrm{GA}_{3}$ which displays one of their accumulated peaks at this specific bent cotyledon embryo stages (Hu et al., 2018).

Thus in accordance with the delayed embryo developmental shift of the Atcrk5-1 embryos, it can be explained that the reduced level of the bioactive GAs play a critical role here. To support this we measured the level of total gibberllic acid (GA) concentration in the medium size siliques (S5-S6-S7) by a quantitative GA antibody based ELISA kit assay. We could notice decreased levels of GA when measured in these medium sized siliques of Atcrk5-1 mutant compared to wild type siliques of the the same age (Figure 37A). In the case of Atcrk5-1 mutant embryos we could notice the decreased capacity of the first peak of GA accumulation, leading to problems with the embryo differentiation in case of later 
stages of embryo developmental process as observed. In addition to the delayed embryo development in the Atcrk5-1 mutant we could also observe the shorter SAM-RAM axis distance in the bent cotyledon embryos arised from inflorescence siliques of the mutant as compared to wild type embryos. Further we investigated the effect of the exogenous supply of gibberellic acid (GA) action during embryogenesis in case of Atcrk5-1 mutant from early inflorescence of greenhouse-grown plants. On exogenous GA treatment we could observe the restoration of the SAM-RAM axis distance shortage phenotype of the mutant embryos to the wild type level at S11-S12 stages (Figure 37B). In addition to this, the more opened hook phenotype of Atcrk5-1 mutant found in S11-S12 was also restored to wild type level upon external GA treatment (Figure 37C).

A

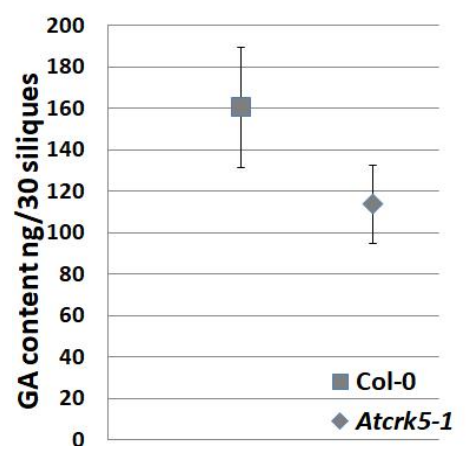

B

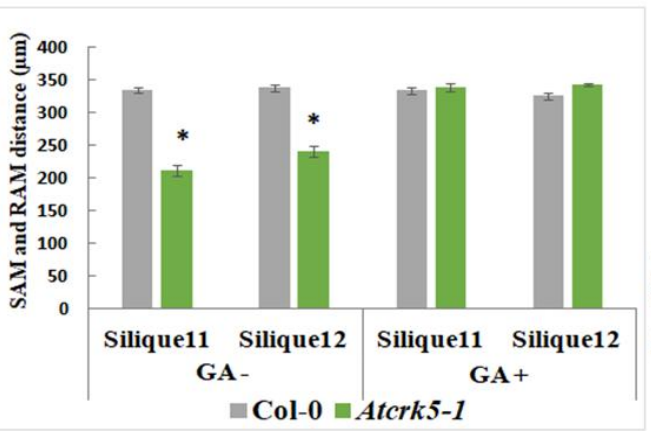

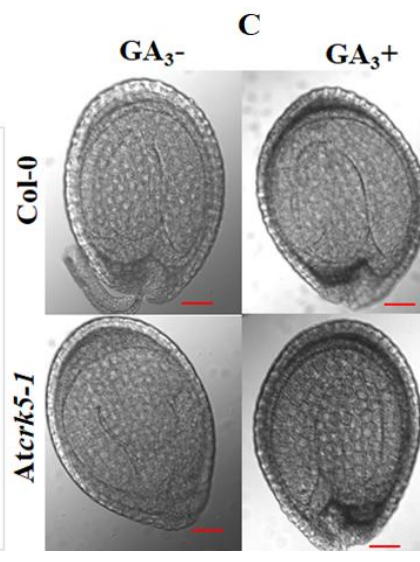

Figure 37: Role of gibberellic acid in development of embryo. (A) Total GA content in medium stage siliques (from S5-S6-S7) in the (Col-0) and mutant (Atcrk5-1), the total GA concentration was determined in septum bearing seeds using GA antibody based ELISA kit after removing the valves. (B) Quantification of embryo axis size differences in siliques S11-S12. Embryo axis from SAM to RAM was measured after treatments of developing inflorescence without and with $20 \mu \mathrm{M} \mathrm{GA}_{3}$ in wild type (Col-0) and Atcrk5-1 mutant plants. Without the exogenous $\mathrm{GA}_{3}$ supply, the Atcrk5-1 embryo shows significantly shorter embryo axis compairing with wild type Col-0 embryo axis. Asterisks $(*)$ depict significant differences between the wild type and mutant embryos (Student's t-test: * $\mathrm{p} \leq 0.05, \mathrm{n}=\min .30$ ). The data presented are the means of two biological repeats. (C) Recovery of the delayed embryo bending phenotype in S11 of the Atcrk5-1 mutant embryos by exogenous $\mathrm{GA}_{3}$ treatment $(\mathrm{n}=\min .30)$. The experiments were repeated for two times with the same results (Scale bars $=100 \mu \mathrm{m}$ ).

Various genes have been known to be involved in development of embryos, so we further investigated the expression levels of the selected genes during embryo development. Polar auxin transporter protein coding genes (PAT) PIN1, PIN4 and PIN7 that are important in auxin efflux transport, the genes of transcription factors $L E C 1, L E C 2$, FUS3 and $A B I 3$ involved in maturation of embryo and its dormancy regulation processes were investigated. Also the genes encoding the family of five homologs of the DELLA proteins that are reported to play a prominent role in GA signaling during embryogenesis (Hu et al., 2018, Salanenka et al., 2018) were selected for gene expression studies. Additionaly to the above selected genes, the expression of the gibberellin 20-oxidases 
(AtGA20ox2 and AtGA20ox3) which are GA biosynthesis enzymes highly expressed during dry and imbibed seeds (Rieu et al., 2008), play a committed role in conversion of the inactive intermediates of GA biosynthesis into its bioactive forms (Hu et al., 2018). Expression of the gibberellin deactivation gene GA2ox4 known to display higher expression levels in 4-7 days after pollination (4-7 DAP) of embryo development (Hu et al., 2018) was also analysed in the study. Gene expression studies were performed on the siliques from different position which specify the days after pollination from inflorescences of wild type (Col-0) and mutant (Atcrk5-1) plants. Siliques were categorized and collected according to their stages of development, like small (S1-S2-S3-S4; 3 DAP), medium (S5S6-S7; 5 DAP) ones which corresponds to early (S1-S4) and medium (S5-S7) embryogenesis stages. In accordance with the decreased levels of GA content observed in medium silique stages in the Atcrk5-1 mutant, we could observe out of the five DELLAs a slightly increased gene expression in RGL1 (Figure 38A) and GAI (Figure 38D) genes, but the expression pattern of the other three $R G L 2, R G L 3$ and $R G A$ (Figure 38B, C and E) genes did not change in the Atcrk5-1 medium siliques when compared to that of the wild type Col-0. In addition to this, the levels of GA20ox2/GA20ox3 gene expression were decreased in case of medium siliques of the Atcrk5-1 (Figure 38F/G). This decreased expression levels indicate that the decline in the GA level during the medium silique stage as described may be the consequence of insufficient conversion of the inactive GA intermediates into their bioactive forms which results in the insufficient GA levels from the embryos arising from medium siliques. Expression levels of the GA2ox4 gene was also found decreased (Figure 38H) in the Atcrk5-1 medium siliques which reports the decreased effect of GA biosynthetic feed back mechanism.

Furthermore the expression patterns of PIN genes encoding polar auxin transporters were analysed. We found PIN1 to be slightly down regulated in the Atcrk5-1 mutant during small and medium size silique stages, inclusive torpedo stage (from S1 till S7, Figure $38 \mathrm{~K})$. Regarding the expression level of PIN4 and PIN7 genes we evidence them to be slightly reduced in case of small silique stage of the Atcrk5-1 mutant (S1-S4, Figure 38I and Figure 38J). So this goes in coordination with the fact that their activity on embryo polarity determination starts from proembryo stages (S1-S4). Furthermore we did not notice any changes in the expression levels at the small silique stage of the transcription factors LEC1, LEC2 and FUS3 which display their main role during late stages of embryogenesis. However in the Atcrk5-1 mutant at the medium size siliques (from S5-S7, Figure 38L,M,N) the expression level of these genes were down regulated. In addition to 
this, the expression level of the other transcription factor gene $A B I 3$ was decreased at the level of medium silique stage in the Atcrk5-1 mutant (Figure 380). As it was reported that the genes like ABI3, FUS3 and LEC2 are known to be the important regulators of the seed maturation processes. The ABI3, FUS3 expression decrease probably can be the consequence of the delayed embryogenesis in Atcrk5-1 mutant.
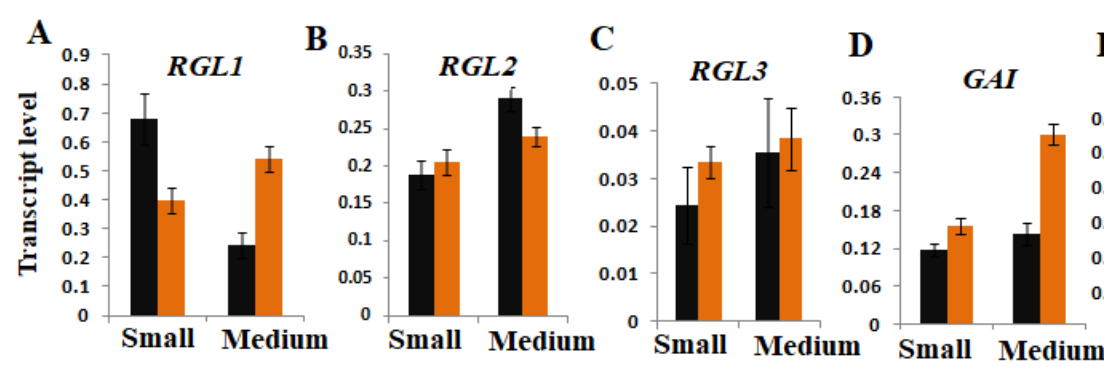

\section{$\mathbf{E}$}
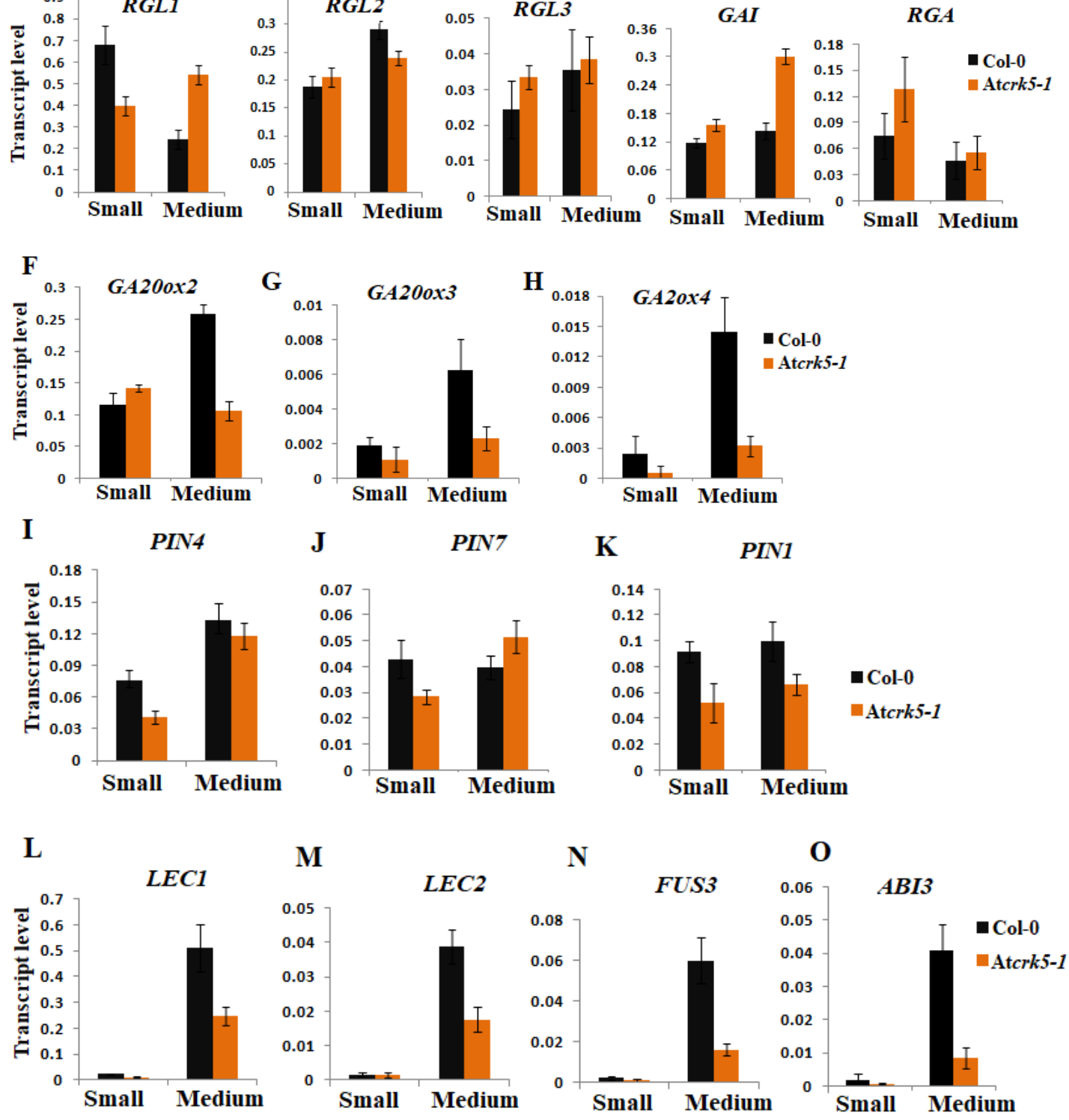

Figure 38: Expression of various genes during embryo development. Expression level of various genes involved in morphogenesis and maturation of embryo at different siliques stages. Transcript levels were determined in small siliques (S; S1-S4, 2DAP), medium siliques (M, S5-S7, 3DAP). The expression of DELLA genes $R G L 1$ (A), RGL2 (B), RGL3 (C), GAI (D) and RGA (E), the gibberellin biosynthesis gene GA20ox2 (F) and GA20ox3 (G), the GA catabolism enzyme coding gene GA2ox4 (H), the polar auxin transport (PAT) protein coding genes PIN4 (I), PIN7 (J) and PIN1 (K) and the LEC1 (L) and LEC2 (M), FUS3 $(\mathrm{N})$ and $A B I 3(\mathrm{O})$ were measured. Ubiquitin $1(U B Q-1)$ is used as an internal control. The values are means $( \pm)$ SD of two independent biological repeats. Primers used are listed in the Supplementary Figure S1. 
It is already explained that a fine crosstalk between auxin and GA is important and GA plays a pivotal role in proper auxin transport in Arabidopsis (Willige et al., 2011). In the Atcrk5-1 mutant we could find low levels of GA in embryos during globular/ heart/torpedo embryo stages (S5-S7) and this period of embryo development (from silique S5 till silique S11) is the time when embryos go through its rapid expansion process in the case of wild type plants (Hu et al., 2018).

\subsubsection{Distribution of auxin and its transporters in the Atcrk5-1 mutant}

In addition to GA level measurement we were also interested about the distribution of auxin levels in the Atcrk5-1 mutant and we verified it by using DR5::GFP marker line (Ottenschläger et al., 2003) (a synthetic promoter which responds to auxin presence) introduced into wild type (Figure 39A) and Atcrk5-1 mutant (Figure 39B). We analysed its expression in different stages of embryo development in both of the lines. During confocal laser scanning microscopy (CLSM), we could evidence decreased intensity of DR5::GFP signal in the Atcrk5-1 embryos from 8-cell stage until walking stick stages of the embryos (Figure 39B).

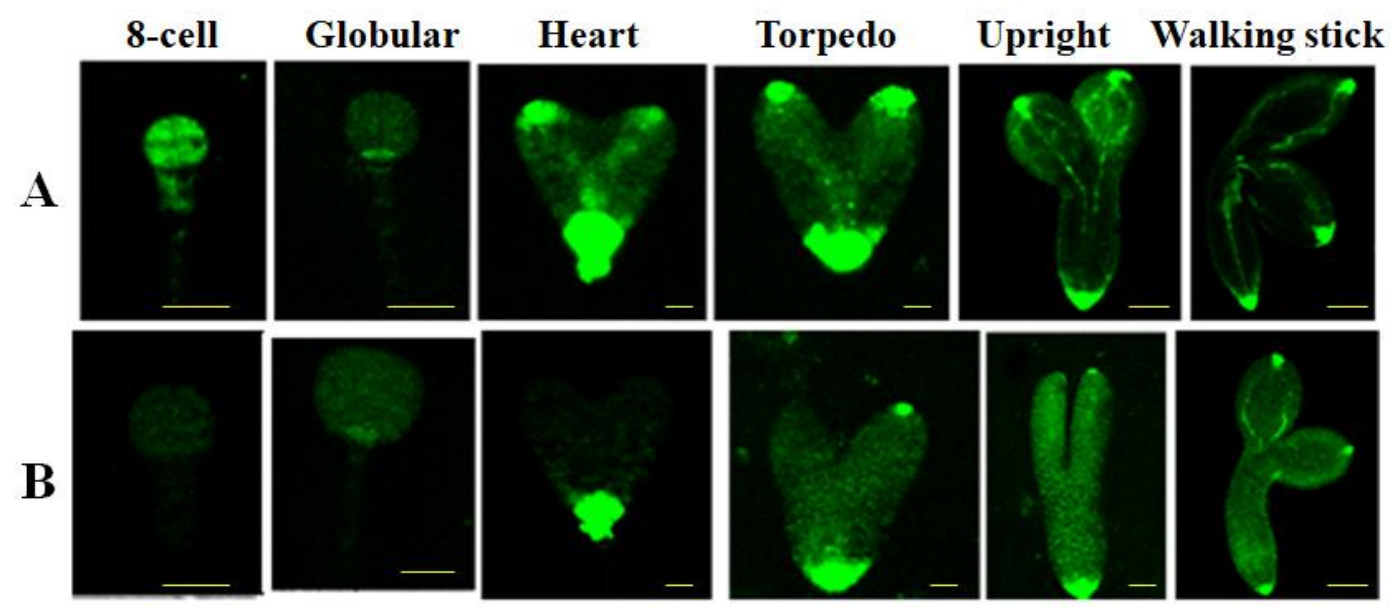

Figure 39: DR5::GFP as an auxin sensor for measurement of auxin levels in wild type Arabidopsis and mutant Atcrk5-1 embryos at various developmental stages. Embryo development in Col-0 (A) and in the Atcrk5-1 (B) mutant. Decreased auxin levels can be noticed in every embryo developmental stages of Atcrk5-1 mutant. (Scale bars: 8-cell to Torpedo= $15 \mu \mathrm{m}$; Upright/Walking stick= $100 \mu \mathrm{m}$ ).

In the case of embryo development, expression of various PIN genes occurs and the auxin efflux transporter proteins in the embryos display the asymmetrical plasma membrane localization determining the further role in directional flow of the cell-to-cell auxin transport in plant cells (Wisniewska et al., 2006, Adamowski and Friml 2015). In order to rule out the localization pattern of the auxin efflux transporters like PIN1, PIN4 and PIN7 during embryogenesis in Atcrk5-1 mutant, we analyzed their GFP-tagged version 
(PIN1-GFP, PIN4-GFP, and PIN7-GFP) distribution in various developmental stages of embryos. During the early stages of embryogenesis auxin transporters PIN1 and PIN7 play role in the developmental process (Friml et al., 2003). When comparing the PIN1-GFP localization patterns in case of Atcrk5-1 mutant with wild type Col-0 embryos (Figure 40) from 8-cell stage untill the walking stick embryo stage, we could observe significant decrease of the GFP signal intensity in Atcrk5-1 mutant (see also heat maps, Supplementary Figure S9). On the heat maps, the intensity of the GFP signal correlates with the coloration where red colour represents the highest and black colour the lowest signal intensity.

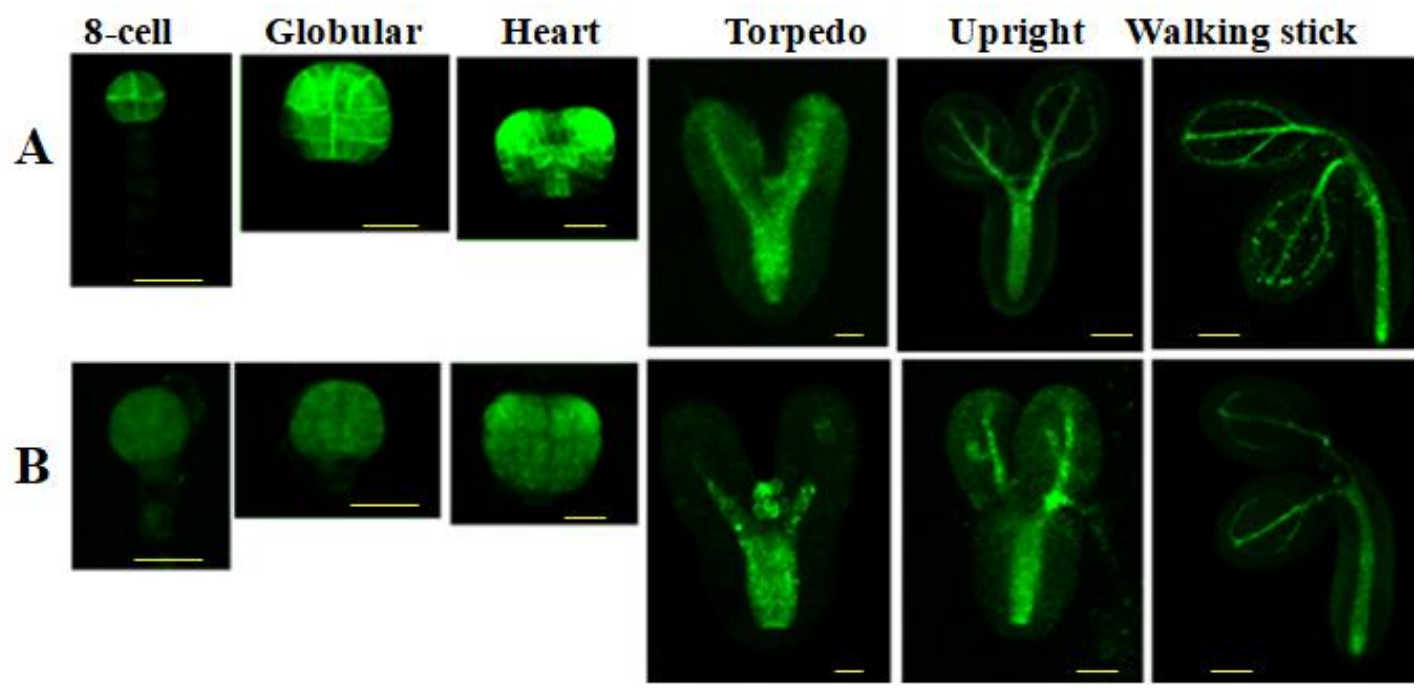

Figure 40: Abundance of PIN1-GFP transporter in wild type (A) and in Atcrk5-1 mutant (B) embryos during embryogenesis. Images show the GFP signal location of PIN1-GFP, suggesting the less abundance of PIN1-GFP transporter in Atcrk5-1 mutant than the control Col-0 embryos. (Scale bars: 8-cell to Torpedo= $15 \mu \mathrm{m}$; Upright/Walking stick= $100 \mu \mathrm{m})$.

Other member of PIN transporter family like PIN7 is known to initially possess defined localization towards the apical side of suspensor cells and also at the 32-cell stage (globular embryo stage) (Friml et al., 2003). So we analysed the PIN7-GFP lines to observe its distribution in Atcrk5-1 mutant during embryogenesis. We could notice the strong decrease of PIN7-GFP signal intensity in the Atcrk5-1 embryos at every analysed embryo stages (Figure 41). 


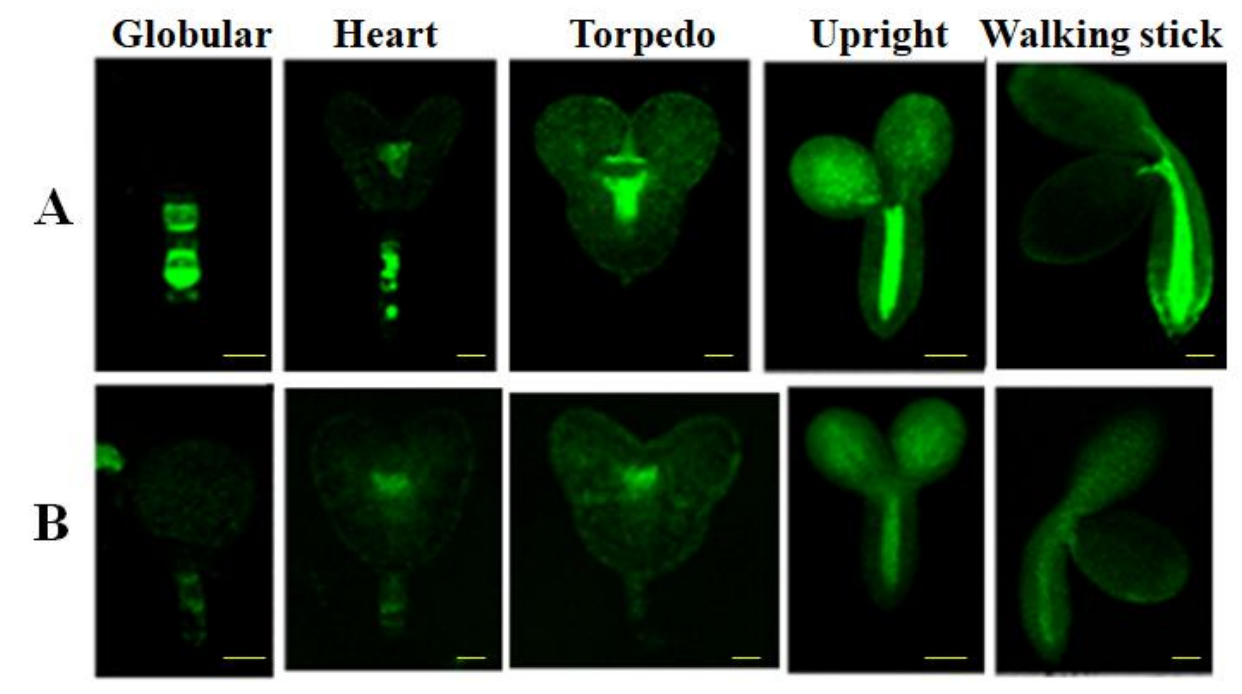

Figure 41: Abundance of the PIN7-GFP transporter in Arabidopsis embryos at various develomental stages. (A) PIN7-GFP intensity during embryo development in wild type Col-0 background and (B) in the mutant Atcrk5-1. Note decreased abundance of PIN7-GFP in Atcrk5-1 mutant. (Scale bars: Globular to Torpedo $=15 \mu \mathrm{m}$; Upright $/$ Walking stick $=100 \mu \mathrm{m}$ ).

The other important PIN proteins expressed during the particular stages of embryogenesis, PIN4 protein usually appears at the globular stage of the embryogenesis specifically located at the descendants of hypophysis and to provascular initials of root meristem (Friml et al., 2003). On proceeding with the localization of PIN4-GFP pattern in case of wild type and Atcrk5-1 embryos, we could report the similar changes in the abundance of PIN4-GFP likewise we observed in case of PIN1-GFP and PIN7-GFP in Atcrk5-1 embryos (Figure 42).

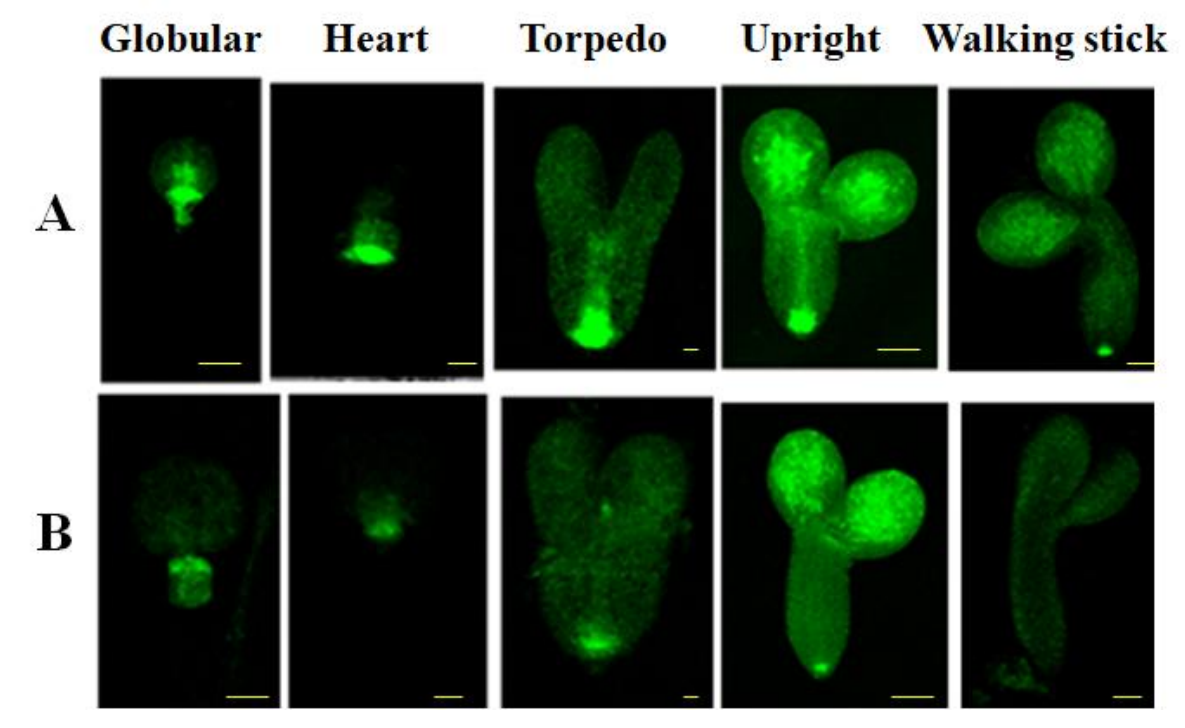

Figure 42: Abundance of PIN4-GFP transporter in wild type Arabidopsis and mutant Atcrk5-1 embryos at several develomental stages. PIN4-GFP intensity during embryo development in wild type Col-0 (A) and (B) in the mutant Atcrk5-1. Note the decreased PIN4-GFP abundance in every embryo developmental stage of Atcrk5-1 mutant. (Scale bars: Globular to torpedo= $15 \mu \mathrm{m}$; Upright/Walking stick= $100 \mu \mathrm{m}$ ). 
After observing the differences in the abundance of PIN1-GFP, PIN4-GFP and PIN7-GFP in the case of Atcrk5-1 mutant, we further evaluated the levels of PIN3 proteins from the various embryo stages. So we were interested in investigating PIN3 transporter abundance in the wild type and Atcrk5-1 embryos during embryogenesis. But in the case of PIN3-GFP we could not notice any considerable prominent differences in GFP intensity pattern and abundance in analysed embryos of the Atcrk5-1 mutant when we compared with wild type embryos (Supplementary Figure S10).

Besides auxin efflux transporters various influx carriers working in coordination with PIN transporters have also been observed to play an important role during embryogenesis. Further we compared the GFP pattern of one of the main influx transporter AUX1 tagged with Yellow fluorescent protein (YFP) and we recorded the AUX1::YFP signal in the wild type background (Col-0) and mutant (Atcrk5-1) embryos at various selected developmental stages of embryogenesis (Figure 43). In accordance with the PIN1, PIN4 and PIN7 transporters, here we also noticed a promisable decrease and change in abundance patterns of AUX1::YFP signal in the Atcrk5-1 mutant during various embryo developmental stages. In Atcrk5-1 as we observed the reduction in the SAM-RAM axis distance conferring to the decreased sizes mostly prominent in late stages of embryogenesis. So we can corroborate this reduction in axis can be a cooperated consequence of impaired levels of auxin efflux and influx carriers PINs/AUX1 in the absence of the AtCRK5 protein kinase.

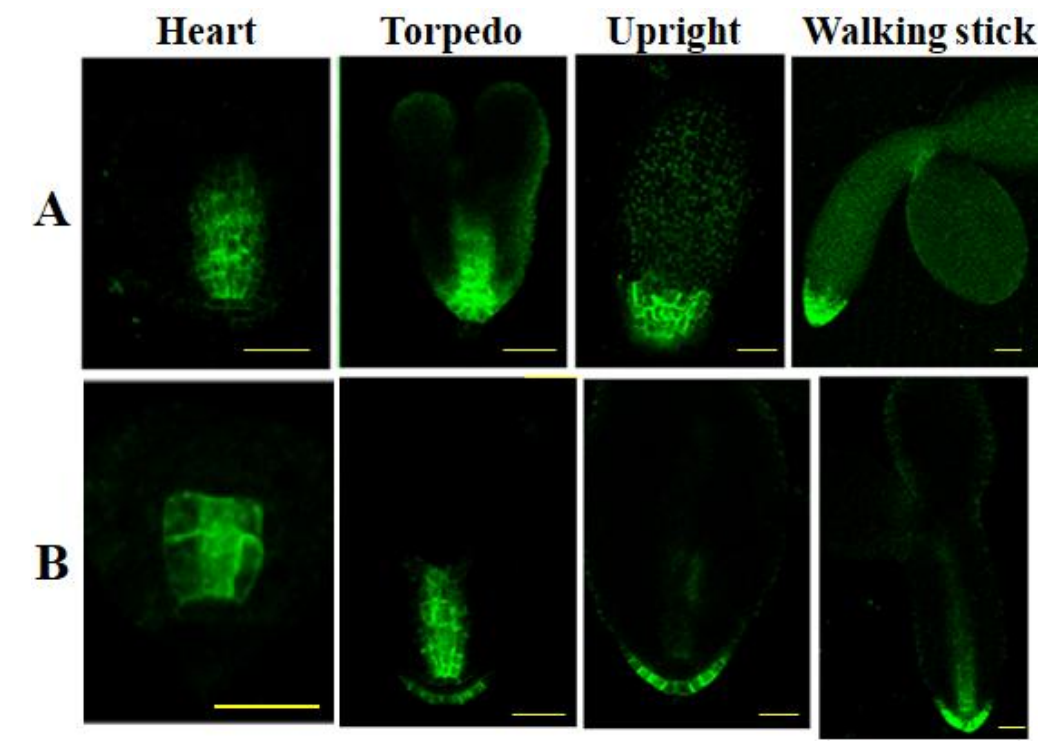

Figure 43: Expression of AUX1::YFP from heart till walking stick stages of embryo development. (A) Abundance of AUX1::YFP during embryo development in wild type (AUX1::YFP + Col-0) and (B) in the mutant (AUX1::YFP + Atcrk5-1). In several embryo developmental stages there is a decrease in abundance of AUX1::YFP expression in the Atcrk5-1 mutant (Scale bars $=100 \mu \mathrm{m})$. 
As previously predicted that the AtCRK protein kinase family displayed highly conserved $\mathrm{N}$-terminal domains including their myristoylation/palmitoylation sites (Lu et al., 2013; Rigó et al., 2013) and later the confirmation of PM localization of the most of AtCRK proteins ( $\mathrm{Lu}$ et al., 2013; Rigó et al., 2013; Baba et al., 2018). We also were interested to find the localization pattern of this protein kinase during the various developmental stages of embryo. On observation of the AtCRK-GFP construct we noticed the GFP signal intensity appeared at every embryo stage and indicated plasma membrane localization of AtCRK5 (see Supplementary Figure S11). This coordinated with our earlier study in agreement with the plasma membrane localization of the AtCRK5 protein kinase (Rigó et al., 2013) and with the similar localization patterns of almost all Arabidopsis AtCRK family members (Baba et al., 2018).

The marked potential feature of PIN transporters is their central hydrophilic loop that separates two hydrophobic domains of about five transmembrane regions (Křeček et al., 2009). The localization and the activation of these various PIN proteins are known to be affected by their phosphorylation events (Křeček et al., 2009; Offringa and Huang, 2013; Luschnig and Vert, 2014; Barbosa et al., 2018). It has previously been reported that AtCRK5 phosphorylates PIN2, the main auxin efflux transporter responsible for controlling the gravitropic response of the Arabidopsis roots upon gravistimulation (Rigó et al., 2013). Also the in vitro phosphorylation of PIN1, PIN2 and PIN3 transporter hydrophilic loop region was already shown to be phosphorylated by AtCRK5 kinase (Lilla Koczka, MSc thesis., 2016; Baba et al., 2019). So in accordance to this we could also notice the decreased abundance of PIN4-GFP and PIN7-GFP in the case of various embryo stages of Atcrk5-1 mutant, which could be correlated with the potential defect in PIN protein stability and localization pattern too. We speculated that based on the high sequence homology between different PIN hydrophilic loops region these two PIN proteins also might be the potent targets of phosphorylation by AtCRK5 kinase. So we tested whether PIN4 and/or PIN7 were also the potential substrates of the AtCRK5 and posses the sites for phosphorylation targets. In order to rule it out we cloned these hydrophilic loop domain regions into the protein expression vector. After transformation and final expression into Escherichia coli cells, we purified the MBP (Maltose Binding Protein) tagged MBP-PIN4HL and MBP-PIN7HL proteins (Figure 44A). We used the first two fraction MBP-PIN4HL/1-2 and MBP-PIN7HL/1-2 for dialysis. After over-night dialysis for the buffer exchange we tested again the protein amount and integrity by using various amount of protein on SDS-PAGE (Figure 44B). 


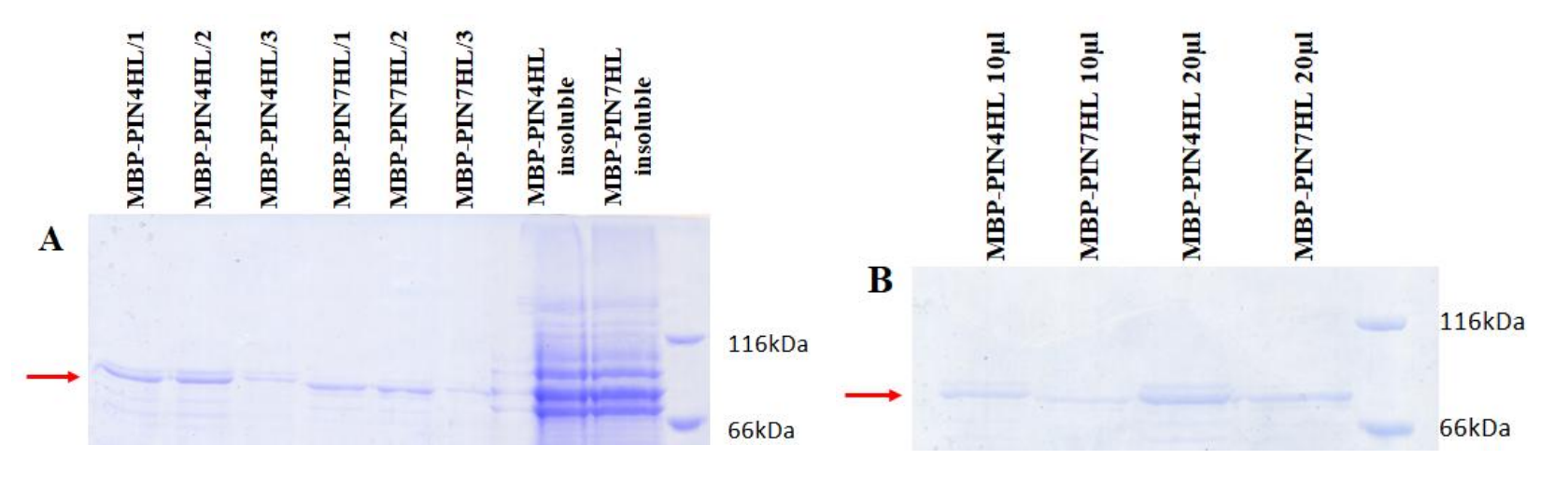

Figure 44: Analysis of the purified MBP-PIN4HL and MBP-PIN7HL proteins. (A) After 10mM Maltose elution, we analyzed 10-10 $\mathrm{l}$ l of protein sample by SDS page, and stained with Coomassie blue. Red arrow shows the purified protein bands. (B) Purified MBP-PIN4HL and MBP-PIN7HL proteins after the dialysis. The red arrow indicate the purified dialyzed protein bands.

Furthermore a non-radioactive kinase assay was performed (by Dr. Gabor Rigo) to determine whether the AtCRK5 kinase could phosphorylate the PIN4HL and/or the PIN7HL loop region. The protein band was visualized by Coomassie staining for the both of the PIN-HL fusions (Figure 45). Using a sterile surgical blade the bands were sliced out and send to the Laboratory of Proteomics Research (http://www.brc.hu/core_proteomics_research.php) to identify the possible in vitro phosphorylation sites.

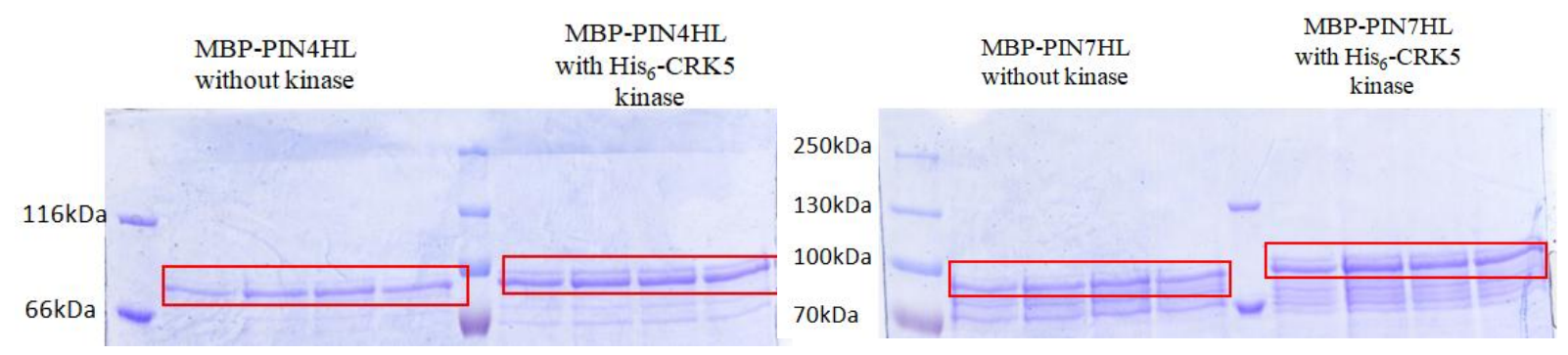

Figure 45: Non-radioactive kinase assay. Figures represent the MBP-PIN4HL and MBP-PIN7HL phosphorylation results. The red rectangle symbolizes the region what was sliced out from the gel for the determination of phosphorylation sites.

We could identify some potential phosphorylation sites also in the case of PIN4 and PIN7 hydrophilic loop. The phosphorylation site in the fusion protein of PIN4 is Ser536, which corresponds to Ser-271 in the database sequence (Figure 46). In case of PIN7 two phosphopeptides were detected in the digest. In one case the site of the modification could be clearly identified, which is Ser-699 in the fusion protein and corresponds to Ser431 in the database sequence. In the other case phosphorylation is on Ser-545 or Ser-546 in 
the fusion protein which corresponds to Ser-277 or Ser-278 in the database sequence (Figure 46). In this study, we could also compare the already identified sites on PIN1HL loop with that of the newly identifed PIN4HL and PIN7HL loop ruling out that these PIN transporters posses specific residues as the potential targets for the phosphorylation by AtCRK5 kinase. Based on these target sites of phosphorylation we suppose that these events are important in the stabilization of the PIN1, PIN4 and PIN7 auxin efflux proteins and maintenance of the their stability. The Atcrk5-1 mutation lead to the decreased abundance of these transporters which can be corelated possibly by the improper phosphorylation event by the AtCRK5 kinase on the transporters.

\begin{tabular}{|c|c|c|c|c|}
\hline Peptide & Protein Mods & Start & End & Score \\
\hline \multicolumn{5}{|l|}{ PIN4HL } \\
\hline LSNFGPADLYSVQS(Phospho)SR & Phospho@536|537 & 523 & 538 & 26,8 \\
\hline LSNFGPADLYSVQS(Phospho)SR & Phospho@533|536 & 523 & 538 & 30,9 \\
\hline LSNFGPADLYSVQS(Phospho)SRGPTPRPSNFEENNAVK & Phospho@533|536|537|541 & 523 & 554 & 41,3 \\
\hline \multicolumn{5}{|l|}{ PIN7HL } \\
\hline LSNFGPADM(Oxidation)YSVQS(Phospho)SR & Oxidation@540;Phospho@545|546 & 532 & 547 & 40,8 \\
\hline LSNFGPADM(Oxidation)YSVQS(Phospho)SR & Oxidation@540;Phospho@545|546 & 532 & 547 & 38,4 \\
\hline LSNFGPADM(Oxidation)YSVQS(Phospho)SR & Oxidation@540;Phospho@545|546 & 532 & 547 & 42,6 \\
\hline LRC(Methylthio)NS(Phospho)TAELNPK & Phospho@699|700 & 695 & 706 & 28,1 \\
\hline C(Methylthio)NS(Phospho)TAELNPK & Phospho@699=16 & 697 & 706 & 25,3 \\
\hline
\end{tabular}
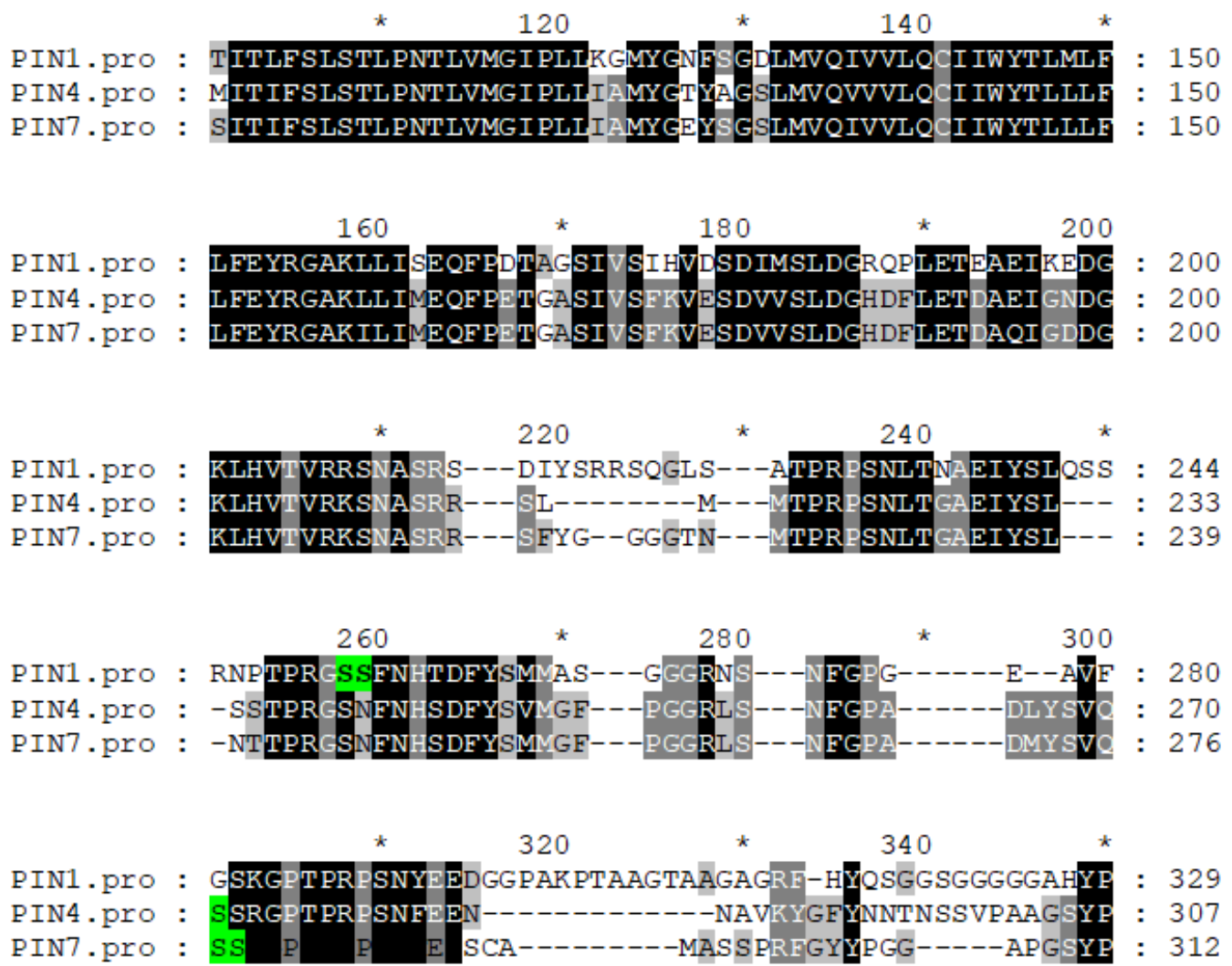
PIN1.pro : APNPGMFS PNTGGGGGTAAKGNAPVV---------GGKRQDGNGRDLHMF : 370 PIN4.pro : APNPEESTGT------GVST KPNKI PKENQQQLQE KDSKASHDAK LHMF : 351 PIN7.pro : APNPEF-----STGNKTGSKAPKENHHH-------VGKSNSNDAKELHMF：350

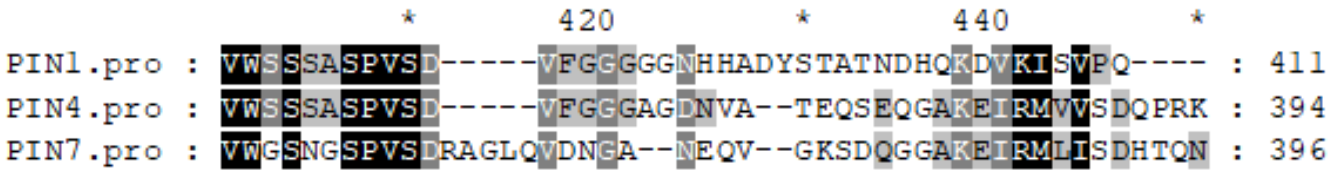

460

480

500

PIN1.pro : ------GNSNDNQYVEREEFSFGNKDDDSKV̄----------TATDGGNNI : 445 PIN4.pro : S-------------GGDDIGGLDSGEGEREIERATA-GLNKMIGSNSTAEL : 430 PIN7.pro : GENRAG--PMNGDYGGE---------EESERVKEV PNGLHKLRCNSTAEL : 435

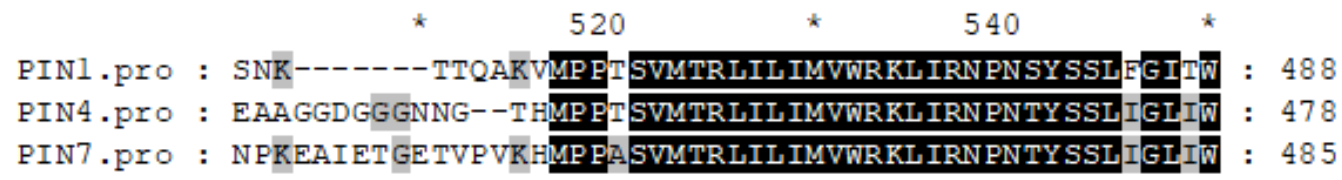

$560 \quad$ * $580 \quad * \quad 600$

PIN1.pro : SLISEKWNIEMPALIAKS IS ILSDAGLGMAMFS LGLEMALNPRIIACGNR : 538

PIN4 .pro : ALVAYRẄHVAMPKILQQS IS ILS DAGLGMAMFS LGLFMALQPKI IACGNS : 528

PIN7 pro : ALVAFRẄDVAMPKIIQQSIS ILSDAGLGMAMFS LGLFMALOPKLIACGNS : 535

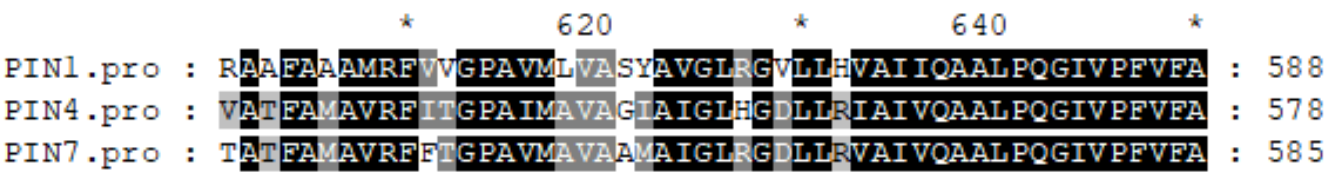

$660 \quad * \quad 680$

PIN1,pro : KEYNVHPDILSTAVI FGMLIALPITLLYYILLGI : 622

PIN4,pro : KEYNVHPTILSTGVI FGMLIALP IT LVYYI LLGL : 612

PIN7.pro : KEYNVHPAILSTGVI FGMLIALP IT LVYYILLGL : 619

Figure 46: The identified AtCRK5 specific phospho-peptide on MBP-PIN4HL and MBP-PIN7HL also its comparison with already detected possible PIN-HL sites, which are highlighted in green. 


\section{DISCUSSION}

\subsection{Functional analysis and subcellular localization of AtCRK protein kinase family}

Protein kinases play a key role in the regulation and modulation of many developmental processes and a series of responses to many environmental or hormonal stimuli. Phosphorylation of proteins is an important regulatory process in signal transduction pathway, which leads to alteration in protein conformation, localization or its stability. Plant protein kinases usually are attributed as the members of certain larger or smaller protein families, possessing the similar primary and secondary protein structure, but can be found variant in their intracellular localization and substrate specificity. Considerable variability can also be observed in the transcriptional regulation of these encoding genes. Despite the similarities, the biological function of these AtCRK proteins can be partially found overlapping, but they can also be different in their function and kinase activity. Hence the characterization of one member of this gene in this protein kinase family does not necessarily provide overall functional information of the whole family of proteins.

CDPK family consist of a group of protein kinases known to represent a large calcium-sensing subfamily of serine/threonine protein kinases reported till date only in the plant kingdom but not in animals (Harper et al., 2004; Simeunovic et al., 2016; Singh et al., 2018). Highly relevant and noteworthy amount of information is available on CDPKs in plants, and are reported to have multiple functions in plants, from the plant growth regulation, development and also in various abiotic and biotic stress responses (Boudsocq and Sheen 2013; Romeis and Herde, 2014). The CDPK-Related Kinase (CRK) protein kinase family is closely related to CDPKs and enough information is available but in contrast to CDPKs very less information is available for AtCRK protein kinases related to their biological functions and subcellular localization (Hrabak et al., 2003; Reddy et al., 2011; Rigó et al., 2013; Wang et al., 2016). Biochemial analysis of phosphorylation status of AtCRKs revealed that various AtCRKs phosphorylate some specific proteins including $\beta$-tubulin (TBB-2), TBB-7 and also some important transcription factors (TFs) such as ethylene response factor 13 (ERF13) (Nemoto et al., 2015). This biochemical characterization of some AtCRKs revealed that they have Tyr-auto/trans-phosphorylation activity and AtCRK2 and AtCRK3, were reported to show high ERF13 Tyrphosphorylation activity. It was shown that specific tyrosine sites Tyr16/Tyr207 in ERF13 
were found to be phosphorylated by AtCRK3 kinase and the Tyr phosphorylation of endogenous TBBs reported in the cells expressing AtCRK2. Mutants Atcrk2 and Atcrk3 displayed a reduction in the Tyr phosphorylation level of TBBs. This biochemical analysis revealed that AtCRKs- additionaly to their Ser/Thr phosphorylation capacity-also posses specific Tyr kinase activity in plants and they are one of the important protein kinases responsible for protein Tyr phosphorylation in Arabidopsis plants. Based on these in vivo studies, these results support the hypothesis that AtCRKs may be involved in protein tyrosine phosphorylation signaling too.

Preliminary subcellular localization study in Arabidopsis protoplasts revealed that AtCRK2 was mainly localized to the plasma membrane and to a lesser degree to nucleus (Nemoto et al., 2015). But no specific marker was used for plasma membrane-localization in this earlier study, thus the possibility of cytoplasmic localization of AtCRK2 could not be excluded. Based on our observations we could rule out that besides the similarities, the biological function of the AtCRK gene family members show variability in their biological function too. In analogy to AtCRK5 the earlier partially characterized protein kinase, we could report that the subcellular localization of most AtCRK proteins plasma membrane localization when analysed either in Arabidopsis protoplasts or in transgenic plants, which overexpress the CRK-eGFPfusions. This plasma membrane localization of AtCRK proteins is shown to be in accordance with the predicted N-terminal myristoylation sites, which is essential for membrane targeting of these proteins (Rigó et al., 2013; Lu and Hrabak, 2013). Further this plasma membrane localization of all AtCRK-eGFP fusion proteins was supported by counterstaining with plasma membrane specific FM4-64 stain and was found to colocalize with GFP fluorescence. Despite the plasma membrane localization of all AtCRK proteins, a peculair endomembrane localization was observed in AtCRK1-eGFP and mild levels in AtCRK7-eGFP, as the GFP-derived fluorescence pattern in root meristematic zone suggests that these AtCRKs might localize at the endomembrane system too. This signal was more prominent and noticeable in case of AtCRK1-eGFP. This was later supported by the cell fractionation and detection of AtCRK1-eGFP in both mitochondria/endomembrane and microsomal fractions. Identical localization pattern was reported for an Arabidopsis sp. choline transporter like1 (CTL1) protein, which is known to regulate the secretory trafficking of auxin transporters PIN1 and PIN3 to control seedling growth (Wang et al., 2017). 
Biologically the functional role of all the AtCRK family members has not been reported much till now, though very limited information is available about this protein kinase family. In this study we also reported the comparative phenotypic analysis of TDNA insertion of Atcrk mutants. We observed that likewise Atcrk5-1 mutant, root geotropic responses of all Atcrk mutants was found to be delayed and also the hypocotyl bending capacities was also observed to have delayed phenotype in these mutants. This delayed root geotropic response can be a associated to be a consequence of altered distribution of PIN2 localization in all Atcrk mutants suggesting that auxin transport is impaired to a certain extent in these mutants as was earlier reported in Atcrk5-1 mutant (Rigó et al., 2013). Many auxin transporters are important to maintain the auxin transport in roots, PIN2 auxin efflux transporter is one of the important transporter and plays a role in basipetal auxin transport in Arabidopsis roots (Müller et al., 1998; Retzer et al., 2017). Earlier it was documented that the delayed gravitropic response in the Atcrk5-1 mutant was the consequence of the improper phosphorylation of the central hydrophilic T-loop of PIN2 in vitro, which is important to maintain its PM association and stability (Rigó et al., 2013). In similarity to impaired phosphorylation of PIN2 in Atcrk5-1, the disturbed localization pattern of PIN2 transporter in other Atcrk mutants can be related to be the consequence of phosphorylation defects (Rigó et al., 2013). During the gravistimulation, all the Atcrk mutants we tested displayed delayed root gravitropic responses upon with variable degrees. We assume that the altered distribution of this PIN2 protein can be a consequence related to the improper phosphorylation of PIN2 protein in the Atcrk mutants, which inturn lead to their decreased stability manifesting the lower PIN2 signal intensities. Future more experimental studies are needed to evaluate the more detailed function of PIN2 phosphorylation by individual AtCRKs.

\subsection{Sensitivity of Atcrk1-1 mutant under continuous illumination}

All of the members of this kinase family might have variable biological functions predicted on their differences in transcriptional regulation, substrate specificity, intracellular localization, protein-protein interactions or both. But in the continuous light conditions most of Atcrk family mutants were found similar to wild type plants in standard growth conditions, except Atcrk1-1 mutant that displayed light hypersensitivity under continuous illumination. Previously the potential role of AtCRK1 in salt tolerance was documented (Tao and Lu, 2013). It was observed that Atcrk1-1 mutant had a decreased tolerance to salt stress during seed germination with higher malondialdehyde (MDA) and 
lower free proline content which indicated its regulatory role in the tolerance to salt stress by changing both MDA and proline levels in Arabidopsis plants (Tao and Lu, 2013). AtCRK1 kinase was also shown to play a regulatory role in the heat tolerance by the interaction and phosphorylation of the heat shock factor A1a (AtHSFA1a) in Arabidopsis sp. (Liu et al., 2008). Based on this previous studies it was suggested that AtCRK1 plays an important role in different environmental stresses including high temperature, extended light and salinity. We could observe that the singlet oxygen $\left({ }^{1} \mathrm{O}_{2}\right)$ content was high in case of Atcrk1-1 mutant, which is a potential indicator of the disturbances in photosynthetic electron transport under continuous light. Oxidative damage can occur during the light stress due to the overreduction of the electron transport chain resulting in the generation of ${ }^{1} \mathrm{O}_{2}$. This increased levels of ${ }^{1} \mathrm{O}_{2}$ generated by the PSII reaction center is shown to interact and further leading damage to the D1 protein of the PSII reaction center that, after the oxidation, has to be replaced by a newly synthesized D1 polypeptide (Vass and Cser, 2009). The increased ${ }^{1} \mathrm{O}_{2}$ content of Atcrk1-1 results in the depleted pigment contents displaying the low carotenoid and chlorophyll content, and also the changes in photosynthetic paprmeters leading to reduced effective PSII quantum yield (ФPSII), lower electron transport rate (ETR) and non-photochemical quenching (NPQ), suggesting that photosynthetic capability in this mutant is decreased. This photosensitivity of Atcrkl-1 was found in similarity to the Arabidopsis thaliana $f l u$ and chlorina(ch1) mutants, which are documented to posses high ${ }^{1} \mathrm{O}_{2}$ content during high light stress.

In analogy to Atcrk1-1, the Arabidopsis mutants $f l u$ and $c h l$ have been reported to have large amounts of ${ }^{1} \mathrm{O}_{2}$ levels without the significant coproduction of other ROS such as hydrogen peroxide (Ramel et al., 2013). Reduction in the parameters of NPQ is characteristic for both the Atcrk1-1 and Atchl mutants (Triantaphylidès et al., 2008). In the case of $f l u$ mutant the necrosis of tissue was noticed during the stress conditions of darklight transition and leading to the activation of a particular stress signaling pathway (op den Camp et al., 2003). We could also observe the tissue necrosis and cell death in the Atcrkl1 mutant in continuous light, which resembles the reaction similar of $f l u$ mutant from dark to light transition or chl mutant to high light stress (Op den Camp et al., 2003; Ramel et al., 2013). Increased ${ }^{1} \mathrm{O}_{2}$ accumulation is reported to have deleterious effect involving the cell death in plants (Mor et al., 2014; Koh et al., 2016). ${ }^{1} \mathrm{O}_{2}$ is not only toxic in nature, but it also acts as a stress signaling molecule in signal transduction network, leading to extensive changes in gene expression, promoting programmed cell death (PCD) or acclimation (Op den Camp et al., 2003; Triantaphylidès et al., 2008; Ramel et al., 2013; 
Koh et al., 2016). In analogy of the light sensitive phenotype of Atcrk1-1 and Atchl mutants and the diminished pigment composition, ROS generation, photoprotective NPQ along with altered photosynthetic parameters suggest the role of AtCRK1 as a suppressor of enhanced ${ }^{1} \mathrm{O}_{2}$ production and also plays role in the the regulation of ${ }^{1} \mathrm{O}_{2}$-triggered cell death and maintenance of cellular redox homeostasis. Further study approach might reveal its in-depth role whether this ${ }^{1} \mathrm{O}_{2}$ generation is a result of decreased chlorophyll, carotenoid biosynthesis or another mechanism that causes the damage to the PSII reaction center.

\subsection{Role of AtCRK5 protein kinase in embryo development}

We also elucidated the other novel role of AtCRK5 protein kinase during embryo development. In the case of Atcrk5-1 mutant we initially noticed the shift in the stages of embryo development at silique stages S5-S11 (starting from globular/early heart embryo stages until the bent cotyledon embryo stage). We related this phenotype of Atcrk5-1 mutant to the consequence of the insufficient levels of bioactive gibberillins. The plant hormone gibberellin is known to play critical role in many forms of plant developmental process, but its elaborated function in the process of embryo development is not much detailed. Bioactive form of gibberellins has been shown to play the role in regulating late embryogenesis in Arabidopsis involving the DELLA proteins in embryos (Hu et al., 2018). During this time the inefficiency of gibberellin biosynthesis or signaling process leads to formation of an abnormal embryo phenotype, characterized by less-developed cotyledons and shortened embryo axis (Hu et al., 2018).

Increased expression patterns peaking during embryo development has been reported to be related to bioactive gibberellins $\mathrm{GA}_{1}$ and $\mathrm{GA}_{3}$ that display enhanced peaks also at the level of late embryo stages. Enhanced synthesis of bioactive gibberellins is found during the late developmental stages of embryos. In coordination this time period is in symmetry with the rapid expansion of the embryos (Hu et al., 2018). The observed decreased levels of bioactive GAs in case of medium sized siliques of Atcrk5-1 mutant can be correlated as a consequence for the shift in the embryo developmental stages in case of Atcrk5-1 mutant. The periodic changes of gibberellic acid involving a two peaks pattern corresponds to particular specific stages of seed development (Locascio et al., 2014). Initial peak in the GA changes correspond to the embryo differentiation when cell growth and expansion occurs (until heart embryo stage, S5-S7), and the second GA maximum peak occurs at the end of the maturation phase of embryos where gibberellins stimulate the proteolytic enzyme activity for mobilizing endosperm resources utilized for germination of matured 
seeds (Locascio et al., 2014). In our results we could observe the insufficiency of Atcrk5-1 mutant embryos to form such first peak of GA involving decreased accumulation which probably exhibited problems with the embryo differentiation in the later embryo developmental stages ( as depicted in Figure 34 and Figure 35). Additionaly to the delayed embryo development in Atcrk5-1 mutant, we observed significant changes in SAM-RAM axis which was shorter in the late stage embryos derived from inflorescence siliques (S1112) compared to wild type embryos, depicting that the ratio of the bioactive GAs was decreased but not completely absent (Figure 37A). When $\mathrm{GA}_{3}$ was supplied exogenously to the growing inflorescence of greenhouse-grown wild type Arabidopsis and Atcrk5-1 mutant plants to further investigate if the phenotype could be rescued during embryogenesis by external $\mathrm{GA}_{3}$ treatment. We observed that it could lead to the restoration of the SAM-RAM axis distance of the mutant embryos to the wild type level at S11-S12 stages (Figure 37B). In addition to this, the delayed bending phenotype of bent cotyledon embryos found in siliques (S10-S12) of Atcrk5-1 mutant could be restored too (Figure 37C).

Previous studies among the hormonal crosstalks have documented a role of fine crosstalk between auxin transport and GA (Frigerio et al., 2006; Willige et al., 2011; Abbas et al., 2013). Also the inefficient auxin transport has been shown to lead to the stabilization of the DELLA repressor RGA (REPRESSOR OF ga1-3) of GA pathway in Arabidopsis (Fu and Harberd, 2003). Auxin is also known to act on GA biosynthesis pathway involving the regulation of the expression of the two key enzymes AtGA20ox1 and AtGA20ox2, which contribute to rate limiting steps of the GA biosynthesis (Frigerio et al., 2006; Hu et al., 2018). This mechanism of auxin signaling has an impact on GA metabolism by the direct up-regulation of GA20ox (metabolic) and GA2ox (catabolic) genes. This regulatory structure is independent from the feedback regulation which is mediated by GAI and RGA DELLA proteins, and includes Aux/IAA and ARF signaling elements (Frigerio et al., 2006, Locascio et al., 2014). So this fact of network signaling and regulation supports the hypothesis of auxin as a potent hormone in promotion of GA biosynthesis. Defect in auxin transport mechanism in the case of one of the GA biosynthesis and signaling mutant gal was reported to be the cause due to the decrease of PIN2 protein auxin efflux facilitator (Willige et al., 2011). GA deficiency has shown to target the PIN2 proteins for vacuolar degradation and reducing the transport of auxin (Willige et al., 2011). PIN protein levels were rescued at wild type level by adding exogenous GA. It is already explained that the auxin transport involving the PIN transporters interacts with GA pathway through embryo 
cotyledon development and root gravitropism (Willige et al., 2011). It was already reported that during the embryo development there is an interaction of DELLA proteins with LEC1 proteins (Hu et al. 2018). At the later stages of embryo development, the highly biosynthesized bioactive GAs initiate the DELLA degradation to resolve their repression on the transcriptional activity of LEC1 to promote further embryo development (Hu et al., 2018). Among the most of DELLA proteins, RGA is considered the most general form involved in all reported gibberellin responses and is known to interact with LEC1 (Hu et al. 2018).

In Atcrk5-1 mutant we could notice decreased levels of DR5::GFP signal in all embryo developmental stages (Figure 39) revealing the insufficient levels of auxin in the different stages of embryo development. On observation of specific auxin efflux transporter PIN proteins PIN1, PIN4 and PIN7 in the Atcrk5-1 embryos, a strong decrease in their abundance was found predicting that impaired auxin transport in the Atcrk5-1 embryos relates to GA biosynthesis pathway resulting in GA deficiency (Willige et al., 2011). This leads to abnormal embryo phenotype in old siliques with a shortened embryo axis and effecting normal bending of cotyledons (Hu et al., 2018). However, the rescue in these phenotypes was seen on exogenous GA treatment (Figure 37) as demonstrated also by ( $\mathrm{Hu}$ et al., 2018). Our results point out the basic role of GA during sequential development of Arabidopsis embryo phases. Besides the PIN transporters, the role of auxin influx carriers AUX1 and LIKE-AUX1 (LAX1) transporters in embryo shoot and root poles formation has been described. In Arabidopsis thaliana this family of proteins such as AUX1/LAX play a prominent role in the cellular organization of radical tip cell in the mature embryos (Ugartechea-Chirino et al., 2010). AUX/LAX-mediated auxin flux is vital for cellular patterning from early embryogenesis onward in Brassica napus (Robert et al., 2015). The model of the controlled auxin transport in embryo development (Robert et al., 2015) reports that the transportation of auxin from suspensor cells to the tips of cotyledon through protoderm involves the role of PIN1 and LAX1 transporter protein. Then further the auxin is transported from the shoot apical meristem (SAM) to the root apical meristem (RAM), by efflux carriers (PIN1 and PIN4) and influx carriers (AUX1 and LAX1) leading activation of auxin signaling.

In the case of mutant Atcrk5-1, the significant decrease in the levels of specific PIN transporters could lead to the decreased levels of expression pattern of AUX1::YFP in different embryo developmental stages. As it was reported already the auxin influx carriers AUX1/LAX1/LAX2 also determine the prominent role to the specification and identity of 
embryonic root and shoot pole (Ugartechea-Chirino et al., 2010; Robert et al., 2015). So a cooperative role of these influx carriers with PIN auxin efflux transporters is important and any misfunctioning of these auxin influx carriers lead to morphological changes in embryo shape (Robert et al., 2015). It was also reported that the changes in the PIN1 and PIN7 polarity change is in connection with the reverse flow of the auxin during the embryogenesis (Friml et al., 2003, Locascio et al., 2014). In our observation we could report reduced distances in the SAM-RAM axis of the Atcrk5-1 embryos at the end stages and reduced bending of their cotyledon (in S11-S12). This decreased axis in Atcrk5-1 mutant can be explained as a consequence of the misfunctional cooperation of the auxin efflux and influx carriers PINs/AUX1, since here the cellular polarization of embryos dependent on auxin obliges the fine balances of efflux and influx auxin transport mechanisms (Robert et al., 2015). Thus on external supply of the one of the bioactive GAs $\left(\mathrm{GA}_{3}\right)$, a rescue in both phenotypes of the Atcrk5-1 mutant could be observed, indicating role of AtCRK5 protein kinase in fine balancing of the auxin-GA homeostasis during embryogenesis (Locascio et al., 2014).

The asymmetrical distribution of PIN proteins in the plasma membrane is determined by phosphorylation status of these transporters which inturn effects their polar trafficking (Ganguly et al., 2012). Various kinases have been known to control the distribution of the auxin through phosphorylation of PIN-FORMED efflux carriers or of ABC transporters (Barbaso and Schwechheimer, 2014). The D6PROTEIN KINASE and PINOID/WAG AGCVIII kinases are certain types of kinases known to activate the PINmediated auxin efflux transport (Barbosa et al., 2018). Previously it was shown that the AtCRK5 can phosphorylate in vitro the hydrophilic loops of various PIN proteins like PIN1, PIN2 and PIN3 (Lilla Koczka, MSc thesis, 2016; Baba et al., 2019). Based on the peptide analysis data we detected the possible phosphorylation sites in PIN4 and PIN7. We assume that these transporters are also the potent targets of AtCRK5 kinase. This phosphorylation on these PIN proteins leads to their stability and optimal abundance in various embryo stages. Our results suggest that in addition to its regulatory role in root gravitropic responses and hypocotyl bending, AtCRK5 can also govern the embryo development in Arabidopsis through the fine tuning of auxin-GA levels by a coordinative approach and stability of the polar auxin transport (PAT) proteins. 


\section{LIST OF REFERENCES}

Abas L, Luschnig C: Maximum yields of microsomal-type membranes from small amount of plant material without requiring ultracentrifugation. Anal. Biochem., 401:217227(2010)

Abbas M, Alabadí D, Blázquez MA: Differential growth at the apical hook: all roads lead to auxin. Front. Plant Sci., 4:441(2013).

Achard P, Genschik P: Releasing the brakes of plant growth: how GAs shutdown DELLA proteins. J Exp Bot., 60(4):1085-92(2009).

Adamowski M, Friml J: PIN-dependent auxin transport: action, regulation, and evolution. Plant Cell, 27/1, 20-32(2015).

Alonso JM, Stepanova AN, Leisse TJ, Kim CJ, Chen H, Shinn P, Stevenson DK, Zimmerman J, Barajas P, Cheuk R, Gadrinab C, Heller C, Jeske A, Koesema E, et.al., : Genome-wide insertional mutagenesis of Arabidopsis thaliana. Science, 301:653-657(2003).

Alvarado MC, Zsigmond LM, Kovács I, Cséplö A, Koncz C, Szabados LM: Gene trapping with firefly luciferase in Arabidopsis. Tagging of stress-responsive genes. Plant Physiol,.134(1):18-27(2004).

Apel K, Hirt H: Reactive oxygen species: metabolism, oxidative stress, and signal transduction. Annu. Rev. Plant Biol., 55:373-399(2004).

Arabidopsis Genome Initiative: Analysis of the genome sequence of the flowering plant Arabidopsis thaliana. Nature, 408:796-815(2000).

Aro EM, Virgin I, Andersson B: Photoinhibition of Photosystem II. Inactivation, protein damage and turnover. Biochimica et Biophysica Acta (BBA) - Bioenergetics, 1143(2):113-134(1993).

Asada K: Production and scavenging of reactive oxygen species in chloroplasts and their functions. Plant Physiol., 141:391-396(2006).

Baba AI, Andrási N, Valkai I, Gorcsa T, Koczka L, Darula Z, Medzihradszky KF, Szabados L, Fehér A, Rigó G, Cséplő Á: AtCRK5 Protein Kinase Exhibits a Regulatory Role in Hypocotyl Hook Development during Skotomorphogenesis. Int. J. Mol. Sci., 20, 3432(2019).

Baba AI, Rigó G, Andrási N, Tietz O, Palme K, Szabados L, Cséplő A: Striving Towards Abiotic Stresses: Role of the Plant CDPK Superfamily Members. In: Palocz-Andresen M., Szalay D., Gosztom A., Sípos L., Taligás T. (eds) International Climate Protection. Springer, Cham., 99-105(2019).

Baba AI, Rigó G, Ayaydin F, Rehman AU, Andrási N, Zsigmond L, Valkai I, Urbancsok J, Vass I, Pasternak T, Palme K, Szabados L, Cséplő Á: Functional Analysis of the Arabidopsis thaliana CDPK-Related Kinase Family: AtCRK1 Regulates Responses to Continuous Light. Int. J. Mol. Sci.,19:1282(2018).

Barbez E, Kubeš M, Rolčík J, Béziat C, Pěnčík A, Wang B, Rosquete MR, Zhu J, Dobrev PI, Lee Y, Zažímalovà E, Petrášek J, Geisler M, Friml J, Kleine-Vehn J: A novel 
putative auxin carrier family regulates intracellular auxin homeostasis in plants. Nature, 485(7396):119-22(2012).

Barbosa ICR, Hammes UZ, Schwechheimer C: Activation and polarity control of PINFORMED auxin transporters by phosphorylation. Trends in Plant Science, 23(6):523-538(2018).

Barbosa ICR, Schwechheimer C :Dynamic control of auxin transport-dependent growth by AGCVIII protein kinases. Curr Opin Plant Biol., 22:108-115(2014).

Batt S, Wilkens MB, Venis MA: Auxin binding to corn coleoptile membranes: Kinetics and specificity. Planta, 130:7-13(1976).

Bechtold N, Ellis J, Pelletier G: In planta Agrobacterium mediated gene transfer by infiltration of adult Arabidopsis thaliana plants. C. R. Acad. Sci. Paris, Life Sci. 316:1194-1199(1993).

Benková E, Ivanchenko MG, Friml J, Shishkova S, Dubrovsky JG: A morphogenetic trigger: is there an emerging concept in plant developmental biology? Trends Plant Sci., 14(4):189-93(2009).

Bennett M, Marchant A, Green H, May S, Ward S, Millner P, Walker A, Schultz B, Feldmann K: Arabidopsis AUX1 gene: a permease-like regulator of root gravitropism. Science, 273:948-950(1996).

Bhalerao RP, Bennett MJ: The case for morphogens in plants. Nat Cell Biol., 5(11):93943(2003).

Bienert GP, Schjoerring JK, Jahn TP: Membrane transport of hydrogen peroxide. Biochim Biophys Acta, 1758:994-1003(2006).

Biljana Stangeland and Zhian Salehian: An improved clearing method for GUS assay in Arabidopsis endosperm and seeds. Plant Molecular Biology Reporter, 20(2):107114(2002).

Blancaflor E, Masson PH: Plant gravitropism. Unraveling the ups and downs of a complex process. Plant Physiology, 133:1677-1690(2003).

Boudsocq M, Sheen J: CDPKs in immune and stress signaling. Trends Plant Sci., 18:3040(2012).

Braybrook SA, Harada JJ: LECs go crazy in embryo development. Trends Plant Sci., 13:624-30 (2008).

Chen JG, Ullah H, Young JC, Sussman MR, Jones AM: ABP1 is required for organized cell elongation and division in Arabidopsis embryogenesis. Genes Dev., 15(7):90211(2001).

Chen T, Fluhr R: Singlet Oxygen Plays an Essential Role in the Root's Response to Osmotic Stress. Plant Physiol., 177(4):1717-1727(2018). 
Cheng S, Willmann M, Chen H, Sheen J: Calcium Signaling through Protein Kinases. The Arabidopsis Calcium-Dependent Protein Kinase Gene Family. Plant Physiology, 129(2):469-485(2002).

Choudhury S, Panda P, Sahoo L, Panda SK: Reactive oxygen species signaling in plants under abiotic stress. Plant Signal Behav., 8:e23681(2013)

Coego A, Brizuela E, Castillejo P, Ruíz S, Koncz C, del Pozo JC, Piñeiro M, Jarillo JA, Paz-Ares J, León J; TRANSPLANTA Consortium: The TRANSPLANTA collection of Arabidopsis lines: a resource for functional analysis of transcription factors based on their conditional overexpression. Plant J., 77(6):944-53(2014).

Czechowski T, Stitt M, Altmann T, Udvardi MK, Scheible WR: Genome-Wide Identification and Testing of Superior Reference Genes for Transcript Normalization in Arabidopsis. Plant Physiol., 139:5-17(2005).

Darwin C: The power of movement in plants. London: John Murray Publishers(1880).

David W, Meinke J, Michael C, Caroline D, Steven D, Rounsley MK: Arabidopsis thaliana: A Model Plant for Genome Analysis. SCIENCE, 282(5389):662-682(1998).

Daviere JM, Achard P: A pivotal role of DELLAs in regulating multiple hormone signals. Mol. Plant. 9: 10-20(2016).

Davies PJ: Plant hormones: their nature, occurrence, and functions. In: Davies PJ (ed) Plant hormones. Springer, Netherlands, pp 1-15(2010).

Dharmasiri N, Dharmasiri S, Estelle, M: The F-box protein TIR1 is an auxin receptor. Nature 435: 441-445(2005).

Ditengou FA, Gomes D, Nziengui H, Kochersperger P, Lasok H, Medeiros V, Paponov A, Nagy SK, Nádai TV, Mészáros T, Barnabás B, Ditengou BI, Rapp K, Qi L, Li X, Becker C, Li C, Dóczi R, Palme K: Characterization of auxin transporter PIN6 plasma membrane targeting reveals a function for PIN6 in plant bolting. New Phytol., 217(4):1610-1624(2018).

Dorcey E, Urbez C, Blazquez MA, Carbonell J, Perez-Amador MA: Fertilizationdependent auxin response in ovules triggers fruit development through the modulation of gibberellin metabolism in Arabidopsis. Plant J., 58:318(2009).

Driscoll SP, Prins A, Olmos E, Kunert KJ, Foyer CH: Specification of adaxial and abaxial stomata, epidermal structure and photosynthesis to $\mathrm{CO} 2$ enrichment in maize leaves. J Exp Bot., 57:381-390(2006).

Eberhard S, Finazzi G, Wollman FA: The dynamics of photosynthesis. Annu. Rev. Genet., 42:463-515(2008).

Effendi Y, Jones AM, Scherer GFE: AUXIN-BINDING-PROTEIN1 (ABP1) in phytochrome-B-controlled responses, Journal of Experimental Botany, 64(16):50655074(2013)

Farmer PK, Choi JH: Calcium and phospholipid activation of a recombinant calciumdependent protein kinase (DcCPK1) from carrot (Daucus carota L.). Biochim. Biophys. Acta, 1434:6-17(1999). 
Feraru E and Friml J: PIN Polar Targeting. Plant Physiology, 147(4):1553-1559(2008).

Fleet CM, Sun TP: A DELLAcate balance: the role of gibberellin in plant morphogenesis. Curr Opin Plant Biol., 8(1):77-85(2005).

Flors C, Fryer MJ, Waring J, Reeder B, Bechtold U, Mullineaux P, Nonell S, Wilson MT, Baker NR: Imaging the production of singlet oxygen in vivo using a new fluorescent sensor, Singlet Oxygen Sensor Green. J. Exp.Bot., 57:1725-1734(2006).

Frigerio M, Alabadi D, Perez-Gomez J, Garcia-Carcel L, Phillips AF, Hedden P, Blazquez MA: Transcriptional Regulation of Gibberellin Metabolism Genes by Auxin Signaling in Arabidopsis. Plant Physiol., 142:553-563(2006).

Friml J, Wieten A, Sauer M, Weijers D, Schwarz H, Hamann T, Offringa R, Jürgens G: Efflux-dependent auxin gradients establish the apical-basal axis of Arabidopsis. Nature, 426:13(2003).

Friml J, Wiśniewska J, Benková E, Mendgen K, Palme K: Lateral relocation of auxin efflux regulator PIN3 mediates tropism in Arabidopsis. Nature, 415:806-809(2002b).

Friml J: Auxin transport - shaping the plant. Curr Opin Plant Biol., 6(1):7-12(2003).

Friml J: Subcellular trafficking of PIN auxin efflux carriers in auxin transport. European Journal of Cell biolog, 89(2-3):231-235(2010).

$\mathrm{Fu} \mathrm{X}$ and Harberd N P: Auxin promotes Arabidopsis root growth by modulating gibberellin response. Nature, 421:740-743(2003).

Furumoto T, Ogawa N, Hata S, Izui K: Plant calcium-dependent protein kinase-related kinases (CRKs) do not require calcium for their activities. FEBS Lett, 396:147151(1996).

Ganguly A, Sasayama D, Cho HT: Regulation of the polarity of protein trafficking by phosphorylation. Mol Cells., 33(5):423-30(2012).

Geldner N, Friml J, Stierhof YD, Jürgens G, Palme K: Auxin transport inhibitors block PIN1 cycling and vesicle trafficking. Nature, 413(6854):425-8(2001).

Genty B, Briantais JM, Baker NR: The relationship between the quantum yield of photosynthetic electron transport and quenching of chlorophyll fluorescence. Biochim. Biophys. Acta, 990:87-92(1988).

Gill SS, Tutejs N: Reactive oxygen species and antioxidant machinery in abiotic stress tolerance in crop plants. Plant Physiology and Biochemistry, 48(12):909-930(2008).

Goto N, Starke M, and Kranz, A: Effect of gibberellins on flower development of the pinformed mutant of Arabidopsis thaliana. Arabidopsis Information Service, 23:6671(1987).

Halliwell B: Reactive species and antioxidants. Redox biology is a fundamental theme of aerobic life. Plant Physiol., 141(2):312-22(2006).

Hamel LP, Sheen J, Séguin A: Ancient signals: comparative genomics of green plant CDPKs. Trends Plant Sci, 19(2):79-89(2013). 
Harmon AC, Gribskov M, and Harper JF: CDPKs - a kinase for every Ca2+ signal. Trends Plant Sci., 5: 154-159(2000).

Harmon AC, Yoo BC, McCaffery C: Pseudosubstrate inhibition of CDPK, a protein kinase with a calmodulin-like domain. Biochemistry, 14;33(23):7278-87(1994).

Harper JF, Breton G, Harmon A: Decoding $\mathrm{Ca}^{2+}$ signals through plant protein kinases. Anпи Rev Plant Biol, 55:263-288(2004).

Harper JF, Harmon A: Plants, symbiosis and parasites: a calcium signaling connection. Nature Reviews Molecular Cell Biology, 6:555-566(2005).

Harrison BR and Masson PH: ARL2, ARG1 and PIN3 define a gravity signal transduction pathway in root statocytes. Plant J., 53:380-392(2008).

Hegeman AD, Rodriguez M, Han BW, Uno Y, Phillips GN. Jr, Hrabak EM, Cushman JC, Harper JF, Harmon AC, Sussman MR: A phyloproteomic characterization of in vitro autophosphorylation in calcium-dependent protein kinases. Proteomics, 6:36493664(2006).

Hideg E: A comparative study of fluorescent singlet oxygen probes in plant leaves. Cent. Eur. J.Biol., 3: 273-284(2008).

Hohmann-Marriott MF, Blankenship RE: Evolution of Photosynthesis. Annu. Rev. Plant Biol., 62:515-48(2011).

Hrabak EM, Chan C WM, Gribskov M, Harper JF, Choi JH, Halford N, Kudla J, Luan S, Nimmo HG, Sussman MR, Thomas M, Walker-Simmons K, Zhu J, Harmon AC: The Arabidopsis CDPK-SnRK Superfamily of Protein Kinases. Plant Physiology, 132(2):666-680(2003).

Hu Y, Zhou L, Huang M, He X, Yang Y, Liu X, Li Y, Hou X: Gibberellins play an essential role in late embryogenesis of Arabidopsis. Nature Plants, 4:289-298(2018).

Jaakola L, Pirttilä A, Halonen M, Hohtola A: Isolation of high quality RNA from bilberry (Vaccinium myrtillus L.) fruit. Mol Biotechnol., 19:201-203(2001).

Jones AM, Venis MA: Photoaffinity labeling of auxin-binding proteins in maize. Proc. Natl. Acad. Sci., 86:6153-6156(1989).

Karimi M, Inzé D, Depicker A: GATEWAY vectors for Agrobacterium-mediated plant transformation. Trends Plant Sci., 7:193-195(2002).

Kepinski S, Leyser O: (2005). The Arabidopsis F-box protein TIR1 is an auxin receptor. Nature 435: 446-451(2005).

Kleine VJ, Friml J: Polar targeting and endocytic recycling in auxin-dependent plant development. Annu. Rev. Cell Dev. Biol., 24:447-73(2008).

Klepikova AV, Kasianov AS, Gerasimov ES, Logacheva MD and Penin, AA (2016), A high resolution map of the Arabidopsis thaliana developmental transcriptome based on RNA-seq profiling. Plant J, 88:1058-1070(2016). 
Klughammer C, Schreiber U: Complementary PS II quantum yields calculated from simple fluorescence parameters measured by PAM fluorometry and the Saturation Pulse method. PAM Application, 1:27-35(2018).

Koczka L: Determination of the Arabidopsis thaliana CRK5 protein kinase phosphorylation sites on the PIN1, PIN2 and PIN3 hydrophilic loop region. MSc thesis, Szegedi Tudományegyetem(2016) http://diploma.bibl.u-szeged.hu/65945/

Koh E, Carmieli R, Mor A, Fluhr R: Singlet Oxygen-Induced Membrane Disruption and Serpin-Protease Balance in Vacuolar-Driven Cell Death. Plant Physiol., 171(3):161625(2016).

Koncz C, Martini N, Szabados L, Hrouda M, Bachmair A, Schell J: Specialized vectors for gene tagging and expression studies. In Plant Molecular Biology Manual, Gelvin, S., Schilperoort, B., Eds., Springer, Dordrecht, B2, pp. 1-22(1994).

Koncz C, Németh K, Rédei GP, Schell J: T-DNA insertional mutagenesis in Arabidopsis. Plant Molecular Biology, 20(5):963-976(1992).

Koornneef M, Jorna ML, Brinkhorst-Van Der Swan DL, Karssen CM: The isolation of abscisic acid (ABA) deficient mutants by selection of induced revertants in nongerminating gibberellin sensitive lines of Arabidopsis thaliana (L.) heynh. Theor. Appl. Genet., 61:385-393(1982).

Křeček P, Skůpa P, Libus J, Naramoto S, Tejos R, Friml J, Zažímalová E: The PINFORMED (PIN) protein family of auxin transporters. Genome Biology, 10(12):249(2009).

Krieger-LA: Singlet oxygen production in photosynthesis. J. Exp. Bot., 56:337-346(2005).

Laloi C and Havaux M: Key players of singlet oxygen-induced cell death in plants. Frontiers in Plant Science. 6:39(2015).

Leclercq J, Ranty B, Sanchez-Ballesta M, Li Z, Jones B, Jauneau A, Pech J, Latché A, 1 Ranjeva R, Bouzayen M: Molecular and biochemical characterization of LeCRK1, a ripening asso-240 ciated tomato CDPK-related kinase. J. Exp. Bot., 56:25-35(2005).

Lee S, Cheng H, King KE, Wang W, He Y, Hussain A, Lo J, Harberd NP, Peng J: Gibberellin regulates Arabidopsis seed germination via RGL2, a GAI/RGA-like gene whose expression is up-regulated following imbibition. Genes Dev., 16(5):64658(2002).

Leyser HM, Lincoln CA, Timpte, Lammer C, Turner J, Estelle M: Arabidopsis auxinresistance gene AXR1encodes a protein related to ubiquitin-activating enzyme E1, Nature, 364, pp. 161-164(1993).

Li RJ, Hua W, Lu YT: Arabidopsis cytosolic glutamine synthetase AtGLN1;1 is a potential substrate of AtCRK3 involved in leaf senescence. Biochem Biophys Res Commun., 342:119-126(2006).

Li Z, Wakao S, Fischer BB, Niyogi KK: Sensing and responding to excess light. Annu. Rev. Plant Biol., 60, 239-260(2009). 
Lichtenthaler HK, Buschmann C: Chlorophylls and carotenoids: measurement and characterization by UV.VIS spectroscopy. Current Protocols in Food Analytical Chemistry ed. New York, NY: John Wiley \& Sons(2001).

Lindzen E, Choi JH: A carrot cDNA encoding an atypical protein kinase homologous to plant calcium-dependent protein kinases. Plant Mol Biol., 28:785-797(1995).

Liu HT, Gao F, Li GL, Han JL, Liu DL, Sun DY, Zhou RG: The calmodulin-binding protein kinase 3 is part of heat-shock signal transduction in Arabidopsis thaliana. Plant J., 55:760-773(2008).

Liu X, Zhang H, Zhao Y, Feng Z, Li Q, Yang HQ, Luan S, Li J, He ZH: Auxin controls seed dormancy through stimulation of abscisic acid signaling by inducing ARFmediated ABI3 activation in Arabidopsis. Proc. Natl. Acad. Sci., 110:1548515490(2013).

Liu Y, Wei H.: Genome-wide identification and evolution of the PIN-FORMED (PIN) gene family in Glycine max. Genome, 60:564-571(2017).

Ljung K: Auxin metabolism and homeostasis during plant development. Development, 140:943-950(2013).

Locascio A, Roig-Villanova I, Bernardi J, Varotto S: Current perspectives on the hormonal control of seed development in Arabidopsis and maize: a focus on auxin. Frontiers in Plant Sciences, 5: 412/ 1(2014).

Löfke C, Luschnig C, Kleine-Vehn J: Posttranslational modification and trafficking of PIN auxin efflux carriers. Mech. Dev., 130:82-94(2013).

Löfke C, Zwiewka M, Heilmann I, Van Montagu MC, Teichmann T, Friml J: Asymmetric gibberellin signaling regulates vacuolar trafficking of PIN auxin transporters during root gravitropism. Proc Natl Acad Sci U S A, 110(9):3627-32(2013).

Lu SX, Hrabak EM: The myristoylated amino-terminus of an Arabidopsis calcium dependent protein kinase mediates plasma membrane localization. Plant Mol. Biol., 82:267-278(2013).

Luerssen H, Kirik V, Herrmann P, Misera S: FUSCA3 encodes a protein with a conserved VP1/ABI3-like B3 domain which is of functional importance for the regulation of seed maturation in Arabidopsis thaliana. Plant J., 15:755-764 (1998).

Luschnig C, Vert G: The dynamics of plant plasma membrane proteins: PINs and beyond. Development, 141(15):2924-38(2014).

Martin MV, Distéfano AM, Zabaleta EJ, Pagnussat GC: New insights into the functional roles of reactive oxygen species during embryo sac development and fertilization in Arabidopsis thaliana. Plant Signal Behav., 8(10): e25714(2013).

Maxwell K, Johnson GN: Chlorophyll fluorescence - a practical guide. J. Exp. Bot., 51/345:659-668(2000).

Meskauskiene R and Apel K: Interaction of FLU, a negative regulator of tetrapyrrole biosynthesis, with the glutamyl-tRNA reductase requires the tetratricopeptide repeat domain of FLU. FEBS Lett., 532:27-30(2002). 
Meskauskiene R. Nater M, Goslings D, Kessler F, op den CR, and Apel K: FLU: a negative regulator of chlorophyll biosynthesis in Arabidopsis thaliana. Proc. Natl. Acad. Sci. U.S.A., 98:12826-12831(2001).

Meyerowitz EM and Somerville CR: Arabidopsis. Cold Spring Harbor Laboratory Press, Cold Spring Harbor, New York 27(1994).

Mittler R, Vanderauwera S, Gollery M, Van BF: Reactive oxygen gene network of plants.Trends Plant Sci., 9(10):490-8(2004).

Möller B, Weijers D: Auxin control of embryo patterning. Cold Spring Harb Perspect Biol., 1(5):a001545(2009).

Mor A, Koh E, Weiner L, Rosenwasser S, Sibony-Benyamini H, Fluhr R: Singlet oxygen signatures are detected independent of light or chloroplasts in response to multiple stresses. Plant Physiol., 165(1):249-61(2014).

Morris DA, Friml J, Zažímalová E: The Transport of Auxins. In Plant Hormones: Biosynthesis, signal transduction, Action! (Davies, P.J., ed.) Kluwer Academic Publishers, pp. 437-470(2010).

Mravec J, Skůpa P, Bailly A, Hoyerová K, Křeček P, Bielach A, Petrášek J, Zhang J, Gaykova V, Stierhof Y-D, Dobrev PI, Schwarzerová K, Rolčík J, Seifertová D, Luschnig C, Benková E, Zažímalová E, Geisler M, Friml J: Subcellular homeostasis of phytohormone auxin is mediated by the ER-localized PIN5 transporter. Nature, 459: 1136-1140(2009).

Müller A, Guan C, Galweiler L, Tanzler P, Huijser P, Marchant A, Parry G, Bennett M, Wisman E, Palme K: AtPIN2 defines a locus of Arabidopsis for root gravitropism control. EMBO J., 17: 6903-6911(1998).

Nemoto K, Takemori N, Seki M, Shinozaki K, Sawasaki T: Members of the Plant CRK Superfamily Are Capable of Trans- and Autophosphorylation of Tyrosine Residues. J. Biol. Chem, 290: 16665-16677(2015).

Noh B, Murphy AS, Spalding EP: Multidrug resistance-like genes of Arabidopsis required for auxin transport and auxin-mediated development. Plant Cell, 13(11):244154(2001).

Normanly J, Slovin JP and Cohen JD: Auxin biosynthesis and metabolism. In: Plant Hormones: Biosynthesis, Transduction, Action! Davies, P. J. (ed.) Kluwer Academic Publishers Ithaca, NY. 2 pp. 36-62(2004).

Offringa R, Huang F: Phosphorylation-dependent trafficking of plasma membrane proteins in animal and plant cells. J Integr Plant Biol., 55(9):789-808(2013).

Oh SA, Lee SY, Chung IK, Lee CH, Nam HG: A senescence-associated gene of Arabidopsis thaliana is distinctively regulated during natural and artificially induced leaf senescence. Plant. Mol. Biol., 30:739-754(1996).

Op den Camp RGL, Przybyla D, Ochsenbein C, Laloi C, Kim C, Danon A, Wagner D, Hideg E, Göbel C, Feussner I, Nater M, Apel K: Rapid induction of distinct stress responses after the release of singlet oxygen in Arabidopsis. Plant Cell, 15:2320 2332(2003). 
Osmond B, Förster B: Photoinhibition: then and now. In Demmig-Adams B, Adams WW III, Mattoo AK., eds. Photoprotection, photoinhibition, gene regulation, and environment. Dordrecht: Springer, 11-22(2008).

Ottenschläger I, Wolff P, Wolverton C, Bhalerao RP, Sandberg G, Ishikawa H, Evans M, Palme K: Gravity-regulated differential auxin transport from columella to lateral root cap cells. Proceedings of the National Academy of Sciences, 100(5):2987-299(2003).

Pan X, Chen J, Yang Z: Auxin regulation of cell polarity in plants. Current opinion in Plant Biology, 28: 144-153(2015).

Peng J, Carol P, Richards DE, King KE, Cowling RJ, Murphy GP, Harberd NP: The Arabidopsis GAI gene defines a signaling pathway that negatively regulates gibberellin responses. Genes Dev., 11(23):3194-205(1997).

Péret B, Swarup K, Ferguson A, et al. AUX/LAX genes encode a family of auxin influx transporters that perform distinct functions during Arabidopsis development. Plant Cell. (7):2874-85(2012).

Petrásek J, Friml J: Auxin transport routes in plant development. Development, 136:26752688(2009).

Pitzschke A, Forzani C, Hirt H: Reactive oxygen species signaling in plants. Antioxid Redox Signal, 8: 1757-1764(2006)

Podell S, Gribskov M: Predicting N-terminal myristoylation sites in plant proteins. BMC Genomics. 5(1):37(2004).

Powles SB: Photoinhibition of photosynthesis induced by visible light. Annual Review of Plant Physiology, 35:15-44(1984).

Primer3Plus. Available online: http://www.bioinformatics.nl/cgibin/primer3plus/primer3plus.cgi (accessed on 20 October 2017).

Przybyla D, Göbel C, Imboden A, Hamberg M, Feussner I, and Apel K: Enzymatic, but not non-enzymatic, ${ }^{1} \mathrm{O}_{2}$-mediated peroxidation of polyunsaturated fatty acids forms part of the EXECUTER1-dependent stress response program in the flu mutant of Arabidopsis thaliana. Plant J., 54:236-248(2008).

Pucciariello C, Banti V, Perata P: ROS signaling as common element in low oxygen and heat stresses. Plant Physiol Biochem., 59:3-10(2012).

Quint M, Gray WM: Auxin signaling. Curr Opin Plant Biol., 9:448-453(2006).

Ramel F, Ksas B, Akkari E, Mialoundama AS, Monnet F, Krieger-LA, Ravanat JL, Mueller MJ, Bouvier F, Havaux M: Light-Induced Acclimation of the Arabidopsis chlorinal Mutant to Singlet Oxygen. Plant Cell, 25:1445-1462(2013).

Ray PM, Dohrmann U, Hertel R: Specificity of auxin-binding sites on maize coleoptile membranes as possible receptor sites for auxin action. Plant Physiol., 60:585591(1977).

Reddy ASN, Ali GS, Celesnik H, Day IS: Coping with stresses: Roles of calcium- and calcium/ calmodulin-regulated gene expression. Plant Cell, 23:2010-2032(2011). 
Rehman AU, Szabó M, Deak Z, Sass L, Larkum LA, Ralph P, Vass I: Symbiodinium sp. cells produce light-induced intra and extracellular singlet oxygen, which mediates photodamage of the photosynthetic apparatus and has the potential to interact with the animal host in coral symbiosis. New Phytol., 212:472-484(2016).

Renna L, Stefano G, Majeran W, Micalella C, Meinnel T, Giglione C, Brandizzi F: Golgi traffic and integrity depend on N-myristoyl transferase-1 in Arabidopsis. Plant Cell, 25(5):1756-73(2013).

Retzer K, Lacek J, Skokan R, del Genio CI, Vosolsobe S, Lanková M, Malínská K, Konstantinova N, Zažímalová E, Napier RM, Petrášek J, Luschnig C: Evolutionary Conserved Cysteines Function as cis-Acting Regulators of Arabidopsis PINFORMED2 Distribution. Int. J. Mol. Sci., 18: 2274(2017)

Rieu I, Ruiz-Rivero O, Fernandez-Garcia N, Griffiths J, Powers SJ, Gong F, Linhartova T, Eriksson S, Nilsson O, Thomas SG, Phillips AL, Hedden P: The gibberellin biosynthetic genes AtGA20ox1 and AtGA20ox2 act, partially redundantly, to promote growth and development throughout the Arabidopsis life cycle. Plant J., 53(3):488-504(2008).

Rigó G, Ayadin F, Szabados L, Koncz Cs, Cséplő Á: Suspension protoplasts as useful experimental tool to study localization of GFP-tagged proteins in Arabidopsis thaliana. Acta Biol. Szeged, 52:59-61(2008).

Rigó G, Ayaydin F, Tietz O, Zsigmond L, Kovács H, Páy A, Salchert K, Darula Zs, Medzihradszky KF., Szabados L, Palme K, Koncz Cs, Cséplő Á: Inactivation of Plasma Membrane-Localized CDPK-RELATED KINASE5 Decelerates PIN2 Exocytosis and Root Gravitropic Response in Arabidopsis. The Plant Cell, 25:15921608(2013).

Ríos G, Lossow A, Hertel B, Breuer F, Schaefer S, Broich M., Kleinow T, Jásik J, Winter J, Ferrando A, Farrás R, Panicot M, Henriques R, Mariaux JB, Oberschall A, Molnar G, Berendzen K, Shukla V, Lafos M, Koncz Z, Rédei GP, Schell J, Koncz C: Rapid identification of Arabidopsis insertion mutants by non-radioactive detection of T-DNA tagged genes. Plant J., 32:243-253(2012).

Robert HS, Chulmin Park C, Gutierrez CL, Wójcikowska B, Pěnčík A, Novák O, Chen J, Grunewald W, Dresselhaus T, Friml J, Laux T: Maternal auxin supply contributes to early embryo patterning in Arabidopsis. Nature Plants, 4:548-553(2018).

Robert HS, Grunewald W, Sauer M, Cannoot B, Soriano M, Swarup R, Weijers D, Bennett M, Kim Boutilier K, Friml J: Plant embryogenesis requires AUX/LAX-mediated auxin influx. Development, 142:702-711(2015).

Robyn MP, Li-Sen Y, Narayana M, Benjamin RH, Yan W, Jessica LW, Patrick HM: Gravity Signal Transduction in Primary Roots. Annals of Botany, 96(5):737743(2005).

Romeis T, Herde M: From local to global: CDPKs in systemic defense signaling upon microbial and herbivore attack. Curr Opin Plant Biol., 20:1-10(2014) 
Ron M, Kajala K, Pauluzzi G, Wang D, Reynoso MA, Zumstein K, Garcha J, Winte S, Masson H, Inagaki S, Federici F, Sinha N, Deal RB, Bailey-Serres J, Brady SM: Hairy Root Transformation Using Agrobacterium rhizogenes as a Tool for Exploring Cell Type-Specific Gene Expression and Function Using Tomato as a Model. Plant Physiol., 166:455-469(2014).

Saini S, Sharma I, Kaur N, Pati PK. Auxin: a master regulator in plant root development, Plant Cell Rep., 32:741-757(2013).

Salanenka Y, Verstraeten I, Löfke C, Tabata K, Naramoto S, Glanc M, Friml J: Gibberellin DELLA signaling targets the retromer complex to redirect protein trafficking to the plasma membrane. PNAS, 115(14):3716-3721(2018).

Salehin M, Bagchi R, Estelle M: SCFTIR1/AFB-Based Auxin Perception: Mechanism and Role in Plant Growth and Development. The Plant Cell, 27(1) 9-19(2015).

Sato EM, Hijazi H, Bennett MJ, Vissenberg K, Swarup R: New insights into root gravitropic signaling. Ann Bot., 96(5):737-43(2005).

Sauer M, Kleine-Vehn J: AUXIN BINDING PROTEIN1: the outsider. Plant Cell, 23: 2033-2043(2011).

Scherer GFE: AUXIN-BINDING-PROTEIN1, the second auxin receptor: what is the significance of a two-receptor concept in plant signal transduction?. Journal of Experimental Botany, 62:3339-3357(2011).

Schulz P, Herde M, Romeis T: Calcium-Dependent Protein Kinases: Hubs in Plant Stress Signaling and Development. Plant Physiol., 163, 523-530(2013).

Schwechheimer C: Gibberellin signaling in plants -the extended version. Frontiers in Plant Sci., 2: 107(2012).

Shu K, Zhou W, Chen F, Luo X, Yang W: Abscisic Acid and Gibberellins Antagonistically Mediate Plant Development and Abiotic Stress Responses. Front. Plant Sci., 9:416(2018).

Shumbe L, Chevalier A, Legeret B, Taconnat, L, Monnet F, Havaux M: Cell Death in Arabidopsis under High-Light Stress Is Controlled by OXI1 Kinase. Plant Physiol., 170:1757-1771(2016).

Simeunovic A, Mair A, Wurzinger B, Teige M: Know where your clients are: subcellular localization and targets of calcium-dependent protein kinases. J. Exp. Botany, 67/13:855-3872(2016).

Simon S, Skůpa P, Viaene T, Zwiewka M, Tejos R, Klíma P, Čarná M, Rolčík J, De Rycke R, Moreno I, Dobrev PI, Orellana A, Zažímalová E, Friml J: PIN6 auxin transporter at endoplasmic reticulum and plasma membrane mediates auxin homeostasis and organogenesis in Arabidopsis. New Phytol., 211(1):65-74(2016).

Singh A, Sagar S, Biswas DK: Calcium Dependent Protein Kinase, a Versatile Player in Plant Stress Management and Development. Crit. Rev. Plant Sci., 36:335-352(2018) 
Sreenivasulu N, Wobus U: Seed-development programs: a systems biology-based comparison between dicots and monocots. Annu. Rev. Plant Biol., 64:189-217 (2013).

Stone SL, Kwong LW, Yee KM, Pelletier J, Lepiniec L, Fischer RL, Goldberg RB, Harada JJ: LEAFY COTYLEDON2 encodes a B3 domain transcription factor that induces embryo development. Proc. Natl Acad. Sci., 98:11806-11811 (2001).

Swarup R, Friml J, Marchant A, Ljung K, Sandberg G, Palme K, Bennett M: Localization of the auxin permease AUX1 suggests two functionally distinct hormone transport pathways operate in the Arabidopsis root apex. Genes Dev, 15(20):2648-53(2001).

Swarup R. and Péret B: AUX/LAX family of auxin influx carriers - an overview. Front. Plant Sci, 3:225(2012).

TAIR-stock-CD96https://www.arabidopsis.org/servlet/TairObject?id=3001623306\&type= stock.

Taiz L, Zeiger E: Plant physiology, Fifth edition. Sinauer Associates Inc, 19:546-577, 22:650-658, A3(2010).

Tanaka H, Dhonuske P, Brewer PB and Friml J: Spatiotemporal asymmetric auxin distribution: a means to coordinate plant development. Cell Mol. Life Sci., 63(23):2738-54(2006).

Tao XC, Lu YT: Loss of AtCRK1 gene function in Arabidopsis thaliana decreases tolerance to salt. J Plant Biol., 56:306-314(2013).

Tian Y, Sacharz J, Ware MA, Zhang H, Ruban AV: Effects of periodic photoinhibitory light exposure on physiology and productivity of Arabidopsis plants grown under low light. J Exp Bot., 68(15):4249-4262(2017).

Triantaphylidès C, Krischke M, Hoeberichts FA, Ksas B, Gresser G, Havaux M, Van Breusegem F, Mueller MJ: Singlet oxygen is the major reactive oxygen species involved in photooxidative damage to plants. Plant Physiol., 148:960-968(2008).

Ugartechea-Chirino Y, Swarup YR, Swarup K, Peret B, Morag Whitworth M, Malcolm Bennett M, Bougourd S: The AUX1 LAX family of auxin influx carriers is required for the establishment of embryonic root cell organization in Arabidopsis thaliana. Annals of Botany, 105:277-289(2010)

Valmonte GR, Arthur K, Higgins CM, MacDiarmid RM. Calcium-dependent protein kinases in plants: evolution, expression and function. Plant Cell Physiol., 55:551569(2014).

Vanneste S, Friml J: Auxin: a trigger for change in plant development. Cell, 136(6):100516(2009).

Vass I, Cser K: Janus-faced charge recombination in photosystem II photoinhibition. Trends Plant Sci., 14:200-205(2009).

Viaene T, Delwiche CF, Rensing SA, Friml J: Trends: Origin and evolution of PIN auxin transporters in the green lineage. Plant Sci., 18(1):5-10(2013). 
Vieten A, Sauer M, Brewer PB, Friml J: Molecular and cellular aspects of auxin-transportmediated development. Trends in plant science, 12(4):160-8(2007).

Vieten A, Vanneste S, Wiśniewska J, Benková E, Benjamins E, Beeckman E, Luschnig C, Friml J: Functional redundancy of PIN proteins is accompanied by auxin-dependent cross-regulation of PIN expression. Development, 132:4521-453(2005).

Wabnik K, Govaerts W, Friml J, Kleine-Vehn J: Feedback models for polarized auxin transport: an emerging trend. Mol. BioSyst., 7:2352-2359(2011).

Wagner D, Przybyla D, Op den CR, Kim C, Landgraf F, Lee KP, Würsch M, Laloi C, Mena Nater, Hideg E, Apel K: The genetic basis of singlet oxygen-induced stress responses of Arabidopsis thaliana. Science, 306:1183-1185(2004).

Wang JP, Xu YP, Munyampundu JP, Liu TY, Cai XZ: Calcium dependent protein kinase (CDPK) and CDPK related kinase (CRK) gene families in tomato: genome wide identification and functional analyses in disease resistance. Mol. Genet. Genomics, 291(2):661-676(2016).

Wang Y, Chai C, Valliyodan B, Maupin C, Annen B, Nguyen HT: Genome-wide analysis and expression profiling of the PIN auxin transporter gene family in soybean (Glycine max). BMC Genom., 16:951(2015).

Wang Y, Liang S, Xie QG, Lu YT: Characterization of a calmodulin-regulated $\mathrm{Ca}^{2+}$ dependent-protein-kinase-related protein kinase, AtCRK1, from Arabidopsis. Biochem J., 383(Pt 1):73-81(2004).

Wang Y, Yang L, Tang Y, Tang R, Jing Y, Zhang C, Zhang B, Li X, Cui Y, Zhang C, Shi J, Zhao F, Lan W, Luan S: Arabidopsis choline transporter-like 1 (CTL1) regulates secretory trafficking of auxin transporters to control seedling growth. PLoS Biol,. 28:15(12):e2004310(2017).

Weigel D: Natural Variation in Arabidopsis: From Molecular Genetics to Ecological Genomics. Plant Physiology., 158 (1):2-22(2012).

Weiss D, Ori N: Mechanisms of cross talk between gibberellin and other hormones. Plant Physiol., 144:1240-1246(2007).

Went FW: Reflections and speculations. Annu Rev Plant Physiol., 25:1-26(1974).

West MAL, Yee KM, Danao J, Zimmerman JL, Fischer RL, Goldberg RB, Harada JJ: LEAFY COTYLEDON1 is an essential regulator of late embryogenesis and cotyledon identity in Arabidopsis. Plant Cell, 6:1731-1745 (1994).

Willige BC, Ghosh S, Nill C, Zourelidou M, Dohmann EM, Maier A, Schwechheimer C: The DELLA domain of GA INSENSITIVE mediates the interaction with the GA INSENSITIVE DWARF1A gibberellin receptor of Arabidopsis. Plant Cell, 19:12091220(2007)

Willige BC, Isono E, Richter R, Zourelidou M, Schwechheimer C: Gibberellin Regulates PIN-FORMED Abundance and Is Required for Auxin Transport-Dependent Growth and Development in Arabidopsis thaliana. The Plant Cell, 23:2184-2195(2011). 
Winter D, Vinegar B, Nahal H, Ammar R, Wilson GV, Provant NJ: An "Electronic Fluorescent Pictograph" Browser for Exploring and Analyzing Large-Scale Biological Data Sets. PLoS ONE, 2, E718(2007).

Wisniewska J, Xu J, Seifertova D, Brewer PB, Ruzicka K, Blilou I, Rouquie D, Benkova E, Scheres B, Friml: Polar PIN localization directs auxin flow in plants. Science, 312:883 (2006).

Wolverton C, Ishikawa H, Evans M: The kinetics of root gravitropism: dual motor and sensors. Journal of Plant Growth Regulation, 21:102-112(2002).

Woodward AW and Bartel B: Auxin: regulation, action, and Interaction. Ann. Bot. (Lond.) 95:707-735(2005).

Wu G, Otegui MS, Spalding EP: The ER-localized TWD1 immunophilin is necessary for localization of multidrug resistance-like proteins required for polar auxin transport in Arabidopsis roots. Plant Cell, 22(10):3295-304(2010).

Yamaguchi S: Gibberellin metabolism and its regulation. Annu. Rev. Plant Biol., 59:225251 (2008).

Ye N, Zhang J: Antagonism between abscisic acid and gibberellins is partially mediated by ascorbic acid during seed germination in rice. Plant Signaling and Behavior, 7-5:563565(2012).

Zádníková P, Petrášek J, Marhavý P, Raz V, Vandenbussche F, Ding Z, Schwarzerová K, Morita MT, Tasaka M, Hejátko J, Van-Der SD, Friml J, Benková E: Role of PINmediated auxin efflux in apical hook development of Arabidopsis thaliana. Development, 137:607-617(2010).

Zádnikova P, Smet D, Zhu Q, Van-der SD, Benková E: Strategies of seedlings to overcome their sessile nature: auxin in mobility control. Front. Plant Sci., $6: 218(2015)$.

Zádnikova P, Wabnik K, Abuzeineh A, Gallemi M, Van-Der SD, Smith RS, Inze D, Friml J, Prusinkiewicz P, Benkova E: A model of differential growth-guided apical hook formation in plants. Plant Cell, 28:2464-2477(2016).

Zažímalová E, Křeček P, Skůpa $\mathrm{P}$, Hoyerová K, Petrášek J: Polar transport of the plant hormone auxin-The role of PIN-FORMED (PIN) proteins. Cell.Mol. Life Sci., 64:1621-1637(2007).

Zazímalová E, Murphy AS, Yang H, Hoyerová K, Hosek P. Auxin transporters--why so many?. Cold Spring Harb Perspect Biol., 2(3):a001552(2010).

Zhang H, Wei C, Yang X, Chen H, Yang Y, Mo Y, Li H, Zhang Y, Ma J, Yang J and Zhang X: Genome-wide identification and expression analysis of calcium-dependent protein kinase and its related kinase gene families in melon (Cucumis melo L.). PLoS One, 12(4):e0176352(2017). 
Zhang L, Liu BF, Liang S, Jones RL, Lu YT: Molecular and biochemical characterization of a calcium/ calmodulin-binding protein kinase from rice. Biochem J., 15;368(Pt 1):145-57(2002).

Zhou J and Luo J: The PIN-FORMED Auxin Efflux Carriers in Plants, Int. J. Mol. Sci., 19(9):2759(2018). 


\section{ACKNOWLEDGEMENT}

First and foremost I would like to express my deep sense of regards to my supervisors Dr. Ágnes Cséplö and Dr. Gábor Rigó for providing me an opportunity to work under their benevolent supervision, a mere short word of thanks seems grossly inadequate for the guidance provided by them in this overall project. Then I would like to express my words of gratitude to Dr. László Szabados for his constant encouragement and careful inspection of works, providing me an opportunity to pursue my $\mathrm{PhD}$ in his wonderful laboratory. I also would like to take it as an opportunity to convey my heartfelt acknowledgement to my laboratory colleagues Norbert Andrási, Dr. Laura Zsigmond, Anna Mária Király, Dóra Faragó for their help and support.

Further I would like to convey my sincere appreciation to Dr. Ildikó Valkai, Dr. Ateeq Ur Rehman, Dr. Nitin Labhane, and Dr. Ferhan Ayaydin for their assistance during the part of my $\mathrm{PhD}$ and also for the Dr. Éva Klement for the Mass spectrometry. I would like to express my deep sense of regards to Dr. Csaba Koncz, Prof. Klaus Palme, Dr. Attila Fehér and Dr. Imre Vass for their critical suggestions and reviews. I would also like to thank Dr. Taras Pasternak, Dr. János Urbancsok and Lilla Koczka for their contribution to the project.

I specially would like to thank my all my International friends community here in Szeged, ITC batchmates and my family members for promoting me to do my best. I wish to express my sincere appreciation for their immense support and concern provided to me by my family, my brother and sister for they blessed me to get success in my work. I would like to thank my beloved Mother and Father to whom I would like to dedicate this thesis. I sincerely thank all my past teachers and classmates for giving me wonderful moments during the whole tenure of my education.

Support: This research was supported by OTKA project No. PD115502 and No. PD128055, NKFI project No. NN118089, OTKA grant No. 124828 and GINOP-2.3.2-152016-00001. I would also like to acknowledge for my $\mathrm{PhD}$ funding from the $\mathrm{C} 1$ Ministerial PhD Fellowship of Tempus Public Foundation, Hungary. 


\section{SUMMARY}

This study reports a comparative analysis of the Arabidopsis thaliana CRK genes, including transcription regulation, intracellular localization, and their biological function. Our results show that besides the similarities, there are certain differences in the biological function of the AtCRK gene family members. In analogy with the AtCRK5, most AtCRK proteins displayed plasma membrane localization in protoplast transient expression or in transgenic plants, overexpressing the AtCRK-eGFP fusions. This plasmamembrane localization of AtCRKs is in accordance with their predicted N-terminal myristoylation sites, present in each AtCRK proteins, which is essential for membrane targeting (Rigó et al., 2013; Lu and Hrabak, 2013). Moreover, its two members AtCRK1-eGFP and AtCRK7GFP displayed a peculiar localization pattern as their GFP-derived fluorescence pattern in root meristematic zone suggests that these AtCRKs might localize at the endomembrane system too. This was further supported by cell fractionation and detection of AtCRK1eGFP in both mitochondria/endomembrane and microsomal fractions. Limited information is available for the biological function of the AtCRK protein kinase family. Therefore we performed the analytic study of various T-DNA insertion mutants of this kinase family and it indicated that similarly to the Atcrk5-1 mutant, root and hypocotyl geotropic responses of almost all Atcrk mutants were reduced, few of the members also displayed delay in germination. Altered distribution of the auxin transporter PIN2 in all Atcrk mutants was observed suggesting that auxin transport is impaired in these mutants like the Atcrk5-1 mutant (Rigó et al., 2013). The auxin efflux transporter PIN2 is one of the most important carriers of the basipetal auxin transport in Arabidopsis roots (Müller et al., 1998; Rigó et al., 2013; Zádnikova et al., 2015). All the Atcrk mutants displayed delayed root gravitropic responses of varying degrees upon gravistimulation. The AtCRK5 protein kinase is able to phosphorylate the PIN2 auxin efflux protein in vitro (Rigó et al., 2013). However, further studies are needed to elucidate the precise function of PIN2 phosphorylation by individual AtCRKs.

Most of Atcrk family mutants were similar to wild type plants in standard growth conditions, but Atcrkl-1 displayed light hypersensitivity under continuous illumination. Enhanced singlet oxygen $\left({ }^{1} \mathrm{O}_{2}\right)$ content in case of Atcrkl-1 mutant indicated the potential disturbances in photosynthetic electron transport under continuous light. Changes in the specific photosynthetic parameters suggested that photosynthetic fitness in this mutant is highly impaired. The photosensible phenotype of Atcrkl-1 was found to be analogous to 
Arabidopsis thaliana flu and chlorina (chl) mutants, which are characterized by high ${ }^{1} \mathrm{O}_{2}$ content. Similarity of the Atcrkl-1 and Atchl mutants in light sensitivity, pigment composition, ROS generation, photoprotective NPQ and changed important photosynthetic parameters suggest that AtCRK1 is a suppressor of enhanced ${ }^{1} \mathrm{O}_{2}$ production and can function as a regulator of ${ }^{1} \mathrm{O}_{2}$-triggered cell death.

Embryogenesis in plants requires a fine cooperative hormonal crosstalk and the fine tuning of signals between various hormones like ethylene, gibberellin, brassinosteroid and auxin is important. The directional flow of auxin is important for the embryo development and is maintained by a coordinated effect of various auxin specific transporters such as the PIN efflux, the AUX1/LAX1 influx transporters. In the embryo polarity determination both the efflux and influx auxin transporters are reported to be important in embryo polarity determination. So the important pattern formation during embryo development in Arabidopsis depends on the auxin biosynthesis, transport, and response in the embryo. So this defined embryo patterning in Arabidopsis involve auxin activity and the molecular mechanisms of auxin action during this phase of plant development (Möller and Weijers, 2009). As specified the cellular and tissue specificity of PIN proteins in the process of embryo development, these PIN transporters like PIN1, PIN4, and PIN7 display polar localization and act in coordination to specify the apical-basal axis of the embryo. In AtCRK5 we could evidence its implication in the maintenance of the local auxin concentration during embryo formation and mediate the auxin-GA crosstalk in embryo development. Embryogenesis in the Atcrk5-1 mutant was delayed with decreased SAMRAM axis in late embryo stages accompained with reduced gibberellic acid (GA) content. We could observe the decreased level of auxin using the DR5::GFP construct, as well as the decreased abundance of the polar auxin transport (PAT) proteins PIN1, PIN4 and PIN7 in different developmental stages of Atcrk5-1 mutant embryos. Moreover, AtCRK5 was previously known to phosphorylate in vitro the hydrophylic loops of PIN1, PIN2 and PIN3 which are involved in auxin transport (Lilla Koczka, MSc thesis, 2016, Baba et al., 2019) and we also report here that it also in vitro phosphorylates the two other transporters PIN4 and PIN7. This status of phosphorylation on these specific PIN transporters contributes in the maintenance of PIN protein stability and abundance in different embryo stages. Our recent findings suggest that in addition to regulatory roles in root gravitropic responses and hypocotyl hook bending, AtCRK5 can also govern the embryo development in Arabidopsis through fine tuning of auxin-GA levels by involvement of various polar auxin transport (PAT) proteins. 


\section{ÖSSZEFOGLALÁS}

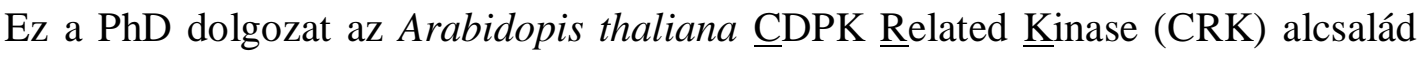
(AtCRK) génjeinek összehasonlitó analizisét mutatja be. A dolgozat magában foglalja az AtCRK gének transcripciós regulációjának, sejten belüli lokalizációjának és biológiai funkciójának vizsgálatát. Eredményeink azt mutatják, hogy - biológiai funkciójukat tekintve - a hasonlóságok mellett bizonyos különbségek is léteznek az AtCRK géncsalád tagjai között. Hasonlóan a korábban már vizsgált AtCRK5 protein kináz intracelluláris localizációjához, az AtCRK család legtöbb tagja szintén plazmamembrán (PM) lokalizációt mutatott mind a protoplast tranziens expressziós kisérletekben, mind a CRKeGFP overexpresszáló transzgénikus növényekben. Az AtCRK protein kináz család tagjainak ez a PM lokalizációja jól magyarázható a fehérjék N-terminális részén található mirisztilációs hely jelenlétével, mivel ez a poszttranszlációs módositási hely megléte teszi lehetővé az AtCRK fehérjék számára a membránokhoz történő kapcsolódását (Rigó et al., 2013, Lu and Hrabak, 2013). Érdekesség, hogy az AtCRK protein kináz család két tagja, az AtCRK1-GFP és az AtCRK7-GFP, a PM lokalizáción kivül még egyéb endomembrán lokalizációt is mutatott a gyökerük merisztématikus zónájában. Az AtCRK1-GFP esetében a differenciális centrifugálási kisérletek tovább támogatták azt a feltevést, hogy ez a protein kináz előfordulhat még a mitokondriális/endomembrán, ill. a mikroszómális frakciókban is.

Az AtCRK protein kináz tagjainak biológiai funkciójáról csak kevés információ elérhető. Ezért elhatároztuk, hogy az AtCRK család tagjainak T-DNS inszerciós mutánsait felhasználva elvégzünk egy összehasonltó funkcionális analizist. Kideritettük, hogy hasonlóan az Atcrk5-1 mutánshoz, az összes többi Atcrk mutáns is késleltetett gravitropikus válasszal rendelkezett a gyökér és hipokotil tesztekben, valamint az Atcrk mutánsok többsége később csirázott, mint a vad tipusú Arabidopsis mag. Az Atcrk mutánsok gyökerében megváltozott az auxin transzporter PIN2 eloszlása, ami az auxin transzport nem megfelelő müködésére utal. Hasonló fenotipust találtunk korábban az Atcrk5-1 mutánsnál (Rigó et al., 2013). Arabidopsis gyökerekben az auxin efflux transzporter PIN2 az egyik legfontosabb tagja a bazipetális auxin transzportnak (Müller et al., 1998; Rigó et al., 2013; Zádnikova et al., 2015). Az AtCRK5 protein kináz in vitro foszforilálta a PIN2 auxin efflux proteint (Rigó et al., 2013). A legtöbb Atcrk mutáns normális fenotipussal rendelkezett a standard növekedési feltételek mellett, azonban az Atcrk1-1 mutáns hiperszenzitivnek bizonyult folyamatos megvilágitási körülmények 
között. A mutánsban megemelkedett szinglet oxigén $\left({ }^{1} \mathrm{O}_{2}\right)$ szintet mértünk, ami a fotoszintetikus elektron transzport zavarára utalt a folyamatos megvilágitás alatt. A specifikus fotoszintetikus paraméterek megváltozása azt sugallta, hogy az Atcrkl-1 mutáns fotoszintetikus fittnessze jelentősen megrendült. Az Atcrk1-1 mutáns fotoszenzitiv fenotipusa nagyon hasonlitott az Arabidopsis thaliana flu és chlorine (chl) mutánsok fenotipusához. Ezek a mutánsok szintén magas ${ }^{1} \mathrm{O}_{2}$ szinttel rendelkeznek. Az Atcrk1-1 és az Atchlmutánsok nagyon hasonlóak, és a fényérzékenységük, a pigment összetételük, a ROS $\left({ }^{1} \mathrm{O}_{2}\right)$ generálásuk, a fotoprotektiv NPQ és más fontos fotoszintetikus paraméterük megváltozása azt sugallja, hogy az AtCRK1 protein kináz egy szupresszora a megemelkedett ${ }^{1} \mathrm{O}_{2}$ produkciónak és ezért feltehetőleg egy regulátora a ${ }^{1} \mathrm{O}_{2}$ indukálta sejthalál folyamatának.

Az Arabidopsis embriók normális fejlődése alatt számos növényi hormon - mint pl. az auxin, etilén, gibberelinek, brasszinoszteroidok - finom kooperativ összjátékát figyelhetjük meg. Az embriók fejlődése szempontjából az auxin irányitott áramlása nagyon fontos szerepet játszik az embriók polaritásának meghatározásában. Ezt az irányitott auxin transzportot a különböző specifikus auxin transzporterek - mint pl. a PIN efflux, az AUX/LAX influx transzporterek - egymással összefüggő müködése tartja fenn az embriógenezis folyamata alatt. Az auxin efflux és influx transzportereknek kitüntetett szerepük van az embrió polaritásának kialakitásában. Tehát az Arabidopsis embrió mintázatának (embryo patterning) kialakulása nagyban függ az auxin bioszintézis, auxin transzport és auxin választól az embriogenezis alatt (Möller and Weijers 2009). Az Arabidopsis embriófejlődés során a PIN1, PIN4 és PIN7 auxin efflux és az AUX1/LAX auxin influx transzporterek játszanak nagy szerepet. Ezek a transzporterek intracellulárisan poláris lokalitációt mutatnak és egymással együtt működve meghatározzák/kialakitják az embrió apikális-bazalis tengelyét. Kimutattuk, hogy az embriófejlődés alatt az AtCRK5 protein kináz működése összefügg a sejten belüli auxin koncentráció fenntartásával, valamint, hogy ez a protein kináz szabályozó szerepet játszik az auxin-gibberellin szint fenntartásában. Az Atcrk5-1 mutáns embriók korai fejlődése során felfedeztünk egy fázis eltolódást a vadtipusú embriófejlődéshez képest, ami a mutáns embriók késői embriógenezisében az embriók SAM-RAM tengelyhosszának lecsökkenését eredményezte. Ezt a fenotipust összefüggésbe tudtuk hozni az embriógenezis alatt létrejövő első gibberellinsav (GA) akkumulációs csúcs nagymértékü lecsökkenésével. Ezenkivül, az Atcrk5-1 mutáns embriók különböző embrionális fejlődési stádiumaiban 
megfigyeltük a poláris auxin transzporter (PAT) PIN1, PIN4, PIN7 és AUX1 proteinek abundanciájának nagymértékü lecsökkenését. Azt is bebizonyitottuk, hogy az AtCRK5 protein kináz - a PIN2 fehérjén kivül - képes in vitro foszforilálni t a PIN4 és PIN7 auxin transzport proteinek hidrofil hurkában levő foszforilációs helyeket is. Eredményeik azt sugallják, hogy az AtCRK5 protein kináz azon felül, hogy reguláló szerepet játszik az Arabidopsis gyökér /szár gravitropikus válaszában, valamint a hipokotil kampó görbülésének szabályozásában, feltehetőleg meghatározó szerepet játszik még az Arabidopsi thaliana embriófejlödésben is, melynek során az auxin-GA szint finom befolyásolásával a poláris auxin transzporter (PAT) proteinek abundancia szintjét határozza meg a PIN proteinek foszforilációja révén. 


\section{PUBLICATION LIST (MTMT ID: 10053163)}

\section{Mandatory peer-reviewed international publications for the fulfilment of doctoral process and on which this thesis is based:}

Baba AI, Rigó G, Ayaydin F, Rehman AU, Andrási N, Zsigmond L, Valkai I, Urbancsok J, Vass I, Pasternak T, Palme K, Szabados L, Cséplö Á: Functional Analysis of the Arabidopsis thaliana CDPK-Related Kinase Family: AtCRK1 Regulates Responses to Continuous Light. Int. J. Mol. Sci.,19, 1282(2018) (I.F: 4.183).

Baba AI, Valkai I, Labhane N, Andrási N, Szabados L, Fehér A, Rigó G and Cséplő A: AtCRK5 protein kinase displays an essential role in embryogenesis of Arabidopsis thaliana. (Manuscript in preparation).

Baba AI, Andrási N, Valkai I, Gorcsa T, Koczka L, Darula Z, Medzihradszky KF, Szabados L, Fehér A, Rigó G, and Cséplő Á: AtCRK5 Protein Kinase Exhibits a Regulatory Role in Hypocotyl Hook Development during Skotomorphogenesis. Int. J. Mol. Sci., 20, 3432(2019) (I.F: 4.183).

\section{Other peer-reviewed international publications}

Andrási N, Rigó G, Zsigmond L, Pérez-Salamó I, Papdi C, Klement E, Pettkó-Szandtner A, Baba AI, Ayaydin F, Dasari R, Cséplö A, and Szabados L: The Arabidopsis Heat Shock Factor A4A is target of MPK4 and regulates responses to combined stresses. Journal of Experimental Botany, erz217(2019) (I.F: 5.360).

Kovács H, Aleksza D, Baba AI, Hajdu A, Király A, Zsigmond L, Toth SZ, Kozma Bognar L, Szabados L: Light control of salt-induced proline accumulation is mediated by ELONGATED HYPOCOTYL 5 in Arabidopsis. Frontiers in Plant Science (2019) (Under revision).

\section{Book chapter:}

Baba AI, Rigó G, Andrási N, Tietz O, Palme K, Szabados L, Cséplö A: Striving Towards Abiotic Stresses: Role of the Plant CDPK Superfamily Members. In: PaloczAndresen M., Szalay D., Gosztom A., Sípos L., Taligás T. (eds) International Climate Protection. Springer, Cham., 99-105(2019). 


\section{Conflict of interest}

We as the corresponding and/or contributing first authors of the below mentioned peer reviewed publication and the unpublished part of this thesis declare that the authors have no conflict of interest related to this study. We also declare that the $\mathrm{PhD}$ candidate Abu Imran Baba worked under our supervision and had a great contribution to the overall results evidenced in this thesis.

Baba AI, Rigó G, Ayaydin F, Rehman AU, Andrási N, Zsigmond L, Valkai I, Urbancsok J, Vass I, Pasternak T, Palme K, Szabados L, Cséplő Á: Functional Analysis of the Arabidopsis thaliana CDPK-Related Kinase Family: AtCRK1 Regulates Responses to Continuous Light. Int. J. Mol. Sci., 19,1282(2018).

Baba AI, Valkai I, Labhane N, Andrási N, Szabados L, Fehér A, Rigó G and Cséplő A: AtCRK5 protein kinase displays an essential role in embryogenesis of Arabidopsis thaliana. (Manuscript in preparation).

Baba AI, Andrási N, Valkai I, Gorcsa T, Koczka L, Darula Z, Medzihradszky KF, Szabados L, Fehér A, Rigó G, and Cséplő Á: AtCRK5 Protein Kinase Exhibits a Regulatory Role in Hypocotyl Hook Development during Skotomorphogenesis. Int. J. Mol. Sci., 20, 3432(2019).

29 August, 2019, Szeged

Dr. Ágnes Cséplő

Sr. Research associate
Dr. Gábor Rigó

Research associate

(Biological Research Centre, HAS, Szeged) 


\section{SUPPLEMENTARY DATA}

Primer for cloning of the CRK genes cDNA's

\begin{tabular}{|l|l|}
\hline CRK1_5'_BAmHI & ACATCggatcctATGGGGATCTGTCATGGAAAACCC \\
\hline CRK1_3'_EcoRV & GATGTgatatccagcAGCTTTTTGTAAGGTCCGAGAAGACACGCC \\
\hline CRK2_5'_Kpnl & ACATCggtacctATGGGAGGCTGTACTTCCAAACCTTC \\
\hline CRK2_3'_Xhol & GATGTctcgaggcTCGCGTTTTCGCTAAAGATTGTCGAGTCGAG \\
\hline CRK3_5'_BAmHI & ACATCggatcctATGGGGCAATGTTACGGGAAGGTTA \\
\hline CRK3_3'_EcoRV & GATGTgatatccagcGCGAGGTCTTGCGTGAGCAGCACGA \\
\hline CRK4_5'_EcoRI & ACATCgaattctATGGGTCATTGTTACAGCCGGAACATC \\
\hline CRK4_3'_EcoRV & GATGTgatatccagcCCTAGGTCTCGAGCTTGAGCTTCGT \\
\hline CRK6_5'_Kpnl & ACATCggtacctATGGGCCACTGTTACAGCCGGA \\
\hline CRK6_3'_EcoRI & GATGTgaattcgcTCTCATTGGTCTCGAGCTCGAGCTTCG \\
\hline CRK7_5'Sall & ACATCgtcgactcATGGGGCTGTGTCATGGGAACC \\
\hline CRK7_3'_EcoRV & GTAAGATATCCTGCCGCTTTCTGCAATGTAC \\
\hline CRK8_5'_Sall & ACATCgtcgactcATGGGAGGCTGTACTTCCAAACCCT \\
\hline CRK8_3'_Xhol & GATGTctcgaggcCCGGGTAGTTTTCCCCGAGGCTCGA \\
\hline
\end{tabular}

qPCR Primer for checking the expression of the CRK genes

\begin{tabular}{|c|c|c|}
\hline CRK1FW & AGTAACGCCATTGTTAGATGAGAA & \\
\hline CRK1REV & AGTGGAGAGGAAAGAAGAAACCA & \\
\hline CRK2FW & GTATCCAGAAAAATCCGACAGG & \\
\hline CRK2REV & CAGCCAAATGAAAAGAGAAAGG & \\
\hline CRK3FW & TCTTCATCAGAACAGAGCAAATAGA & \\
\hline CRK3REV & GAACAAAACAAACGGACGAAA & \\
\hline CRK4FW & CTTGAAATGGAAGTGAAGTCAGG & \\
\hline CRK4REV & CCAGAAGAACAACACCTCTCCA & \\
\hline CRK5-F5 & CAACGAACAATGAAGGCAAAA & Rigó et. al (2013) \\
\hline CRK5-R6 & GATCTCGCCGGAGTCTTCTT & Rigó et. al (2013) \\
\hline CRK6FW & CAAGACAAAAACATCGACTGAAAA & \\
\hline CRK6REV & GGTTCCTCTGAAAACAAAATGCT & \\
\hline CRK7FW & TAAGACATTCTGATGGGAAGCTAAGT & \\
\hline CRK7REV & ATCTAATTCTCTTCTACGCTTTCTGC & \\
\hline CRK8FW & CGGTGAAGATAACGAAAACAAAC & \\
\hline CRK8REV & ACATTTCCAATACCAATCCAAGA & \\
\hline GAPDH2 -FW & AATGGAAAATTGACCGGAATGT & \\
\hline GAPDH2 -Rev & CGGTGAGATCAACAACTGAGACA & \\
\hline
\end{tabular}

qPCR Primer for test the expression of the CRK genes in T-DNA mutants

\begin{tabular}{l|l|}
\hline CRK1_T-DNA_qPCR_5' & CGCATGAACTAAAGATTCCATCTG \\
\hline CRK1_T-DNA_qPCR_3' & GCTCCCGAAGATACGCTAAC \\
\hline CRK2_T-DNA_qPCR_5' & TGGGTATACTTGCTCTGCTAAG \\
\hline CRK2_T-DNA_qPCR_3' & TTCACTTCTCTTCTCACATCCTC \\
\hline CRK3_T-DNA_qPCR_5' & GGATCATCCTATTGCTGTCAAGA \\
\hline CRK3_T-DNA_qPCR_3' & CTCCCTGCGAACATCTTCAA \\
\hline CRK4_T-DNA_qPCR_5' & ACCGGCATGCTTCGATTC \\
\hline CRK4_T-DNA_qPCR_3' & GCTTTGGAAAGGGCTAATGTTATATG \\
\hline CRK8_T-DNA_qPCR_5' & ACAGGCTTTATCTGGACATAAGAA \\
\hline CRK8_T-DNA_gPCR_3' & AAGCTCACCACCTTCACATAG \\
\hline
\end{tabular}


Primers used for embryo studies

\begin{tabular}{|l|l|l|l|l|}
\hline GAI_F & CACCTCGGCTTGGAAACTCTC & ABI3_F & GGATTGTGGAATACTCGGTG \\
\hline GAI_R & TGACAAAGGGAAAACAGTAGGATTT & ABI3_R & TCAGGAAGCGTTGGGAGA \\
\hline RGA_F & AATAGTGGCCAAGGTTATCGTG & PIN1-F & TGGAAGACAACCTTTGGAAACT \\
\hline RGA_R & AGTGTGCCAACCCAACATC & PIN1-R & TGAAGCATTAGAACGACGAACA \\
\hline RGL1_F & CAAGCATGTTGTTGGCACTT & PIN4-F & GGCAACGGAACAATCTGAAC \\
\hline RGL1_R & GCAACAAACAACCTTCATTCTCT & PIN4-R & TCACCACCACCTCTAGCATTAC \\
\hline RGL2_F & CTGCGTTTCCAAAGGAAGAG & PIN7-F & AAGGCGGTGCAAAAGAGATT \\
\hline RGL2_R & GTCGGATCCTCTTGCTGCTA & PIN7-R & CATCGGACCAGCTTTGTTTT \\
\hline RGL3_F & TTATCTGGCGGTGGAGATG & LEC1_F & GTTATGGTATGTTGGACCAATCC \\
\hline RGL3_R & GTTTGCCACGCAAGCATTA & LEC1_R & TTCATCTTGACCCGACGAC \\
\hline GA20ox2_F & ACCGAGACTATTTCCGAGGATT & LEC2_F & CGCCATGAATGGAAATTCGG \\
\hline GA20ox2_R & TGTTTGGCATGGAGGATAATG & LEC2_R & CCACGTACGCGTGATCCTGA \\
\hline GA20ox3_F & AGGAGAAGCTGAGCCGTTT & FUS3_F & TGATACTCCCGAAGAAAGCC \\
\hline GA200x3_R & TTCTCCGGGTAATGGTTCAT & FUS3_R & CTATACTTGAAGGTCCAAACGTG \\
\hline GA20x4_F & GGCTCCAAGTGTCCAATTCA & UBQ1_F & CGCAAGTGCTATGCTCGTCTT \\
\hline GA20x4_R & TCCTACATTGACGCAGAAAGC & UBQ1_R & GCCTCAACTGGTTGCTGTGA \\
\hline
\end{tabular}

Primer set to amplify the PIN4 and PIN7 HL loop from cDNA library

P7HL_F_EcoRI_pMALp2 tttgaattcTACAGAGGAGCTAAGATCTTGATCATGGAA

P7HL_R_SaLI_pMALp2 aaaagtcgactatcaACCAATGAGACTAGAGTAA

P4HL_F_EcoRI_pMALp2 tttgaattcTACCGTGGCGCTAAGCTTCTTATT

P4HL_R_SaLI_pMALp2 aaaagtcgactatcaACCGATTAGACTGGAGTAC

Primers used to eliminate the intron sequence from PIN7 in the final clone:

pin7genomi_5': TACTCAAAATGGTGAAAACAAAGCTGGTCCGATGAACGGGGACTATGGCG

pin7genomi_3' CGCCATAGTCCCCGTTCATCGGACCAGCTTTGTTTTCACCATTTTGAGTA

Figure S1: Primer pairs used in this overall study designed by Primer3Plus online software.

TACAGAGGAGCTAAGATCTTGATCATGGAACAGTTTCCAGAGACTGGTGCTTCGATTGTATCGTTCAAAGTTGAGTCCGA TGTTGTTTCTTTAGATGGACAT GATTTTCTT GAAACTGATGCTCAAATCGGT GACGATGGTAAGCTTCATGTTACGGTGA GAAAAT CAAACGCTTCTCGGAGATCTTTTTACGGTGGT GGTGGTACTAATAT GACTCCTCGTCCGTCTAATCTCACCGGA GCTGAGATTTATAGTCTTAACACTACTCCAAGAGGATCTAACTTCAATCATTCTGATTTTTACTCTATGATGGGGTTTCC CGGTGGCCGGCTTTCGAACTTTGGTCCGGCGGATAT GTACTCTGTTCAGTCCTCGAGAGGACCTACTCCTAGACCTTCGA ATTTTGAGGAGAGTTGTGCTATGGCTTCTTCGCCAAGATTCGGGTATTACCCGGGAGGAGCTCCCGGGTCGTACCCAGCT CCTAATCCGGAGTTTTCTACCGGTAATAAAACCGGTAGTAAAGCTCCAAAAGAGAATCATCATCATGTAGGAAAATCAAA TAGTAATGAT GCTAAGGAGCTTCATATGTTTGTTTGGGGATCCAACGGATCACCCGTTTCGGACCGAGCTGGTCTTCAAG TTGATAAT GGAGCCAAT GAACAAGTCGGAAAATCCGATCAAGGCGGTGCAAAAGAGATTCGAAT GTTGATCTCTGATCAT ACTCAAAATGGTGAAAACAARGgttttaactt taaattgtattttttttgtaacatatggacacaaatct caaactg tatttgct taaatttgtagCTGGTCCGATGA.ACGGGGACTATGGCGGGGA_GAAGAGTCGGAGAGGGTAAAGGAAGTGCC TAACGGACTACACAAGCTTCGGTGTAACTCCACAGCAGAGCTAAACCCTAAAGAAGCTATAGAAACGGGTGAAACTGTAC CGGTAAAACATATGCCACCAGCGAGTGTGATGACTCGGCT GATATTGATAATGGTGTGGAGGAAACTCATAAGAAACCCA AACACTTACTCTAGTCTCATTGGTCTCATT

Figure S2: Genomic region of PIN7 containing a small intron sequence (highlighted in yellow) 


\begin{tabular}{|c|c|c|c|c|c|c|c|c|c|c|c|c|c|c|c|}
\hline Tissue & $\begin{array}{c}\text { CRK1 } \\
\text { Expression }\end{array}$ & $\begin{array}{l}\text { SD1 } \\
\text { SD }\end{array}$ & $\begin{array}{c}\text { CRK2 } \\
\text { Expression }\end{array}$ & $\begin{array}{l}\text { SD2 } \\
\text { SD }\end{array}$ & $\begin{array}{c}\text { CRK3 } \\
\text { Expression }\end{array}$ & $\begin{array}{l}\text { SD3 } \\
\text { SD }\end{array}$ & $\begin{array}{c}\text { CRK4 } \\
\text { Expression }\end{array}$ & $\begin{array}{l}\text { SD4 } \\
\text { SD }\end{array}$ & $\begin{array}{c}\text { CRK5 } \\
\text { Expression }\end{array}$ & $\begin{array}{l}\text { SD5 } \\
\text { SD }\end{array}$ & $\begin{array}{c}\text { CRK6 } \\
\text { Expression }\end{array}$ & $\begin{array}{l}\text { SD6 } \\
\text { SD }\end{array}$ & $\begin{array}{c}\text { CRK7 } \\
\text { Expression }\end{array}$ & $\begin{array}{l}\text { SD7 } \\
\text { SD }\end{array}$ & $\begin{array}{c}\text { CRK8 } \\
\text { Expression }\end{array}$ \\
\hline Dry seed & 62.0 & 5.3 & 68.3 & 4.5 & 21.7 & 6.6 & 42.9 & 5.1 & 31.5 & 1.8 & 9.5 & 1.8 & 12.1 & 2.3 & 12.3 \\
\hline Imbibed seed, $24 \mathrm{~h}$ & 52.1 & 16.8 & 194.3 & 11.0 & 46.4 & 3.7 & 101.6 & 5.7 & 91.5 & 4.6 & 15.7 & 3.9 & 52.0 & 10.6 & 77.9 \\
\hline 1st Node & 112.3 & 2.4 & 91.1 & 0.7 & 71.4 & 4.3 & 154.1 & 9.8 & 289.0 & 15.3 & 16.5 & 1.0 & 90.0 & 21.9 & 51.5 \\
\hline Flower Stage 12, Stamens & 130.5 & 8.5 & 140.1 & 7.4 & 71.8 & 3.6 & 61.5 & 4.6 & 307.4 & 11.1 & 12.9 & 1.6 & 64.2 & 12.4 & 25.6 \\
\hline Cauline Leaf & 199.3 & 13.2 & 62.5 & 5.3 & 55.0 & 4.3 & 155.6 & 5.9 & 232.1 & 35.5 & 33.7 & 2.0 & 53.6 & 8.3 & 21.7 \\
\hline Cotyledon & 99.2 & 14.6 & 72.5 & 3.8 & 37.6 & 3.4 & 130.3 & 3.4 & 183.3 & 5.7 & 32.5 & 5.1 & 47.1 & 7.3 & 32.6 \\
\hline Root & 72.3 & 17.6 & 154.9 & 9.7 & 67.2 & 5.9 & 152.8 & 7.8 & 243.9 & 15.2 & 37.7 & 3.7 & 73.2 & 10.6 & 96.1 \\
\hline Entire Rosette After Transition to Flov & 72.6 & 13.5 & 99.4 & 10.4 & 42.7 & 5.4 & 68.4 & 5.8 & 207.9 & 6.3 & 30.2 & 3.8 & 55.2 & 12.9 & 56.2 \\
\hline Flower Stage 9 & 200.0 & 20.6 & 146.8 & 3.0 & 71.9 & 3.4 & 130.8 & 3.3 & 323.7 & 14.2 & 25.6 & 3.5 & 96.1 & 11.5 & 113.9 \\
\hline Flower Stage 10/11 & 178.9 & 11.2 & 172.6 & 10.3 & 71.5 & 3.2 & 133.7 & 12.9 & 366.3 & 24.4 & 23.0 & 1.7 & 80.1 & 13.2 & 105.8 \\
\hline Flower Stage 12 & 126.7 & 8.9 & 164.9 & 8.5 & 71.4 & 3.2 & 112.6 & 2.1 & 388.7 & 36.7 & 23.0 & 0.8 & 79.4 & 3.1 & 106.3 \\
\hline Flower Stage 15 & 102.4 & 10.5 & 157.5 & 14.3 & 61.7 & 2.5 & 141.9 & 10.5 & 588.1 & 27.4 & 28.2 & 2.5 & 66.9 & 6.8 & 60.3 \\
\hline Flower Stage 12, Carpels & 81.4 & 3.5 & 177.0 & 10.4 & 78.3 & 6.3 & 154.0 & 8.6 & 331.1 & 8.6 & 22.0 & 2.0 & 86.9 & 10.6 & 132.2 \\
\hline Flower Stage 12 , Petals & 138.5 & 14.8 & 232.0 & 12.3 & 115.5 & 6.0 & 207.9 & 3.2 & 678.3 & 10.2 & 18.3 & 1.3 & 82.4 & 12.4 & 99.4 \\
\hline Flower Stage 12, Sepals & 119.8 & 3.3 & 114.4 & 9.9 & 60.7 & 6.5 & 123.7 & 4.6 & 417.8 & 10.9 & 29.9 & 2.4 & 59.4 & 11.5 & 33.6 \\
\hline Flower Stage 15, Carpels & 78.6 & 2.6 & 142.9 & 3.2 & 56.7 & 1.5 & 138.4 & 5.2 & 316.8 & 8.9 & 22.1 & 5.1 & 87.5 & 1.9 & 105.3 \\
\hline Flower Stage 15 , Petals & 166.7 & 25.7 & 262.0 & 7.3 & 117.7 & 3.7 & 169.4 & 16.6 & 1213.1 & 36.5 & 32.2 & 2.0 & 74.8 & 6.2 & 39.2 \\
\hline Flower Stage 15, Sepals & 80.4 & 8.9 & 108.4 & 7.4 & 73.3 & 6.2 & 196.7 & 5.2 & 357.2 & 17.3 & 34.6 & 4.1 & 44.3 & 5.0 & 16.0 \\
\hline Flower Stage 15, Stamen & 130.0 & 21.3 & 273.5 & 13.6 & 41.5 & 1.5 & 125.9 & 18.2 & 559.3 & 8.6 & 17.5 & 1.3 & 47.7 & 2.1 & 26.9 \\
\hline Flowers Stage 15, Pedicels & 71.9 & 3.2 & 126.9 & 4.9 & 39.0 & 3.9 & 76.3 & 11.8 & 212.0 & 22.5 & 38.1 & 3.9 & 65.0 & 13.2 & 76.6 \\
\hline Leaf $1+2$ & 80.4 & 12.8 & 122.5 & 8.1 & 45.2 & 3.4 & 67.0 & 9.7 & 137.4 & 14.1 & 31.5 & 3.8 & 64.5 & 6.1 & 52.5 \\
\hline Leaf 7 , Petiole & 72.4 & 19.3 & 86.2 & 4.9 & 42.5 & 2.4 & 107.7 & 10.4 & 206.7 & 19.9 & 19.8 & 3.0 & 68.0 & 9.8 & 40.3 \\
\hline Leaf 7, Distal Half & 74.2 & 21.8 & 85.9 & 9.4 & 43.6 & 3.1 & 139.4 & 4.0 & 125.6 & 12.7 & 28.6 & 3.7 & 61.1 & 8.8 & 22.7 \\
\hline
\end{tabular}

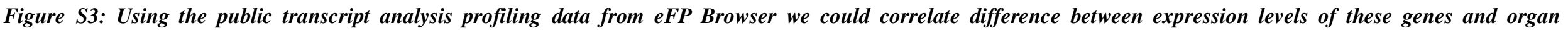
specificities of these genes. 

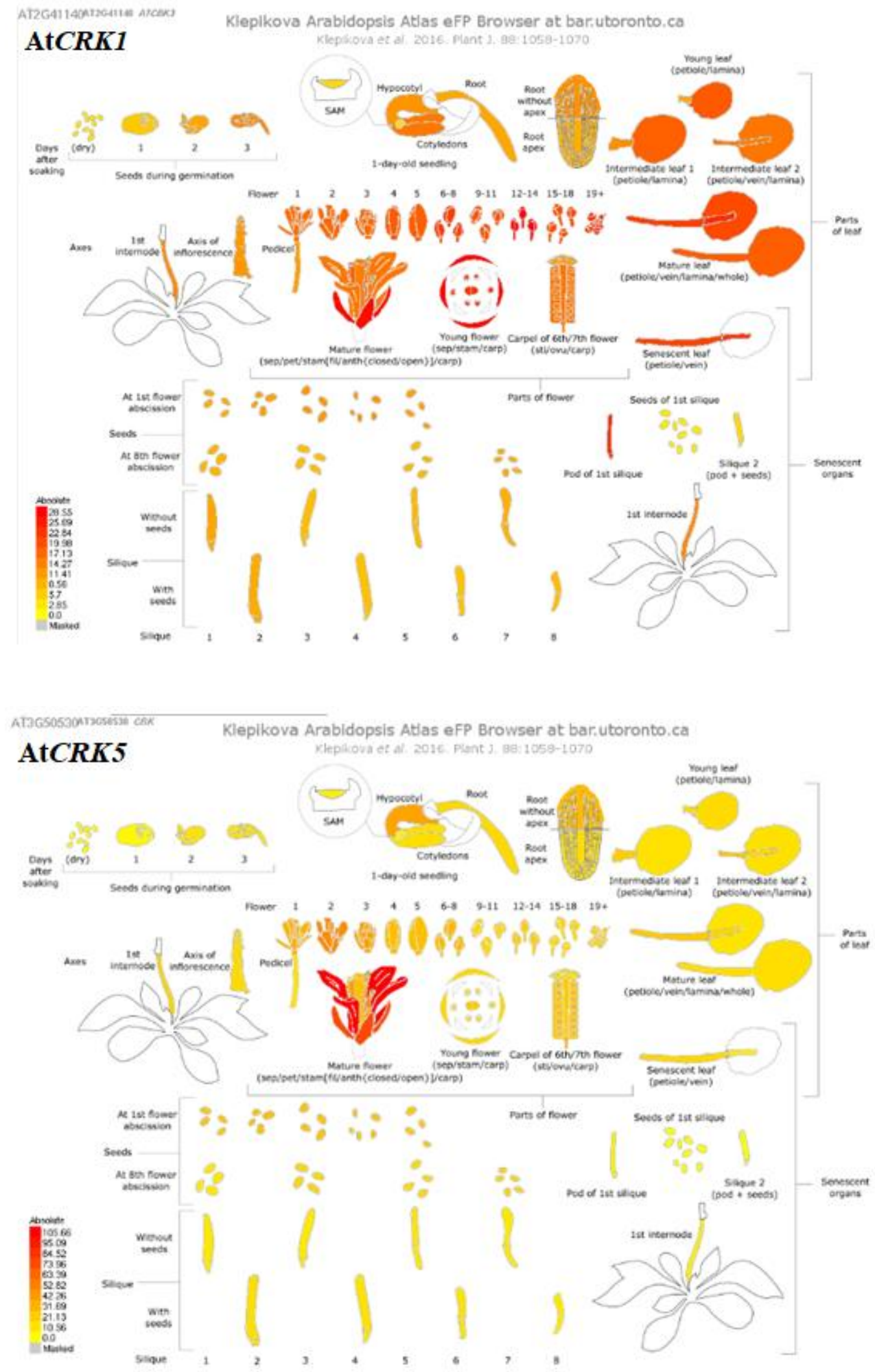

Figure S4: Expression data from a high resolution map of Arabidopsis thaliana developmental transcriptome AtCRK1 and AtCRK5 based on RNA-seq profiling reproduced from eFP Browser (Klepikova et al., 2016) 


\begin{tabular}{|c|c|c|c|}
\hline CRK kinases & AGI & Annotation & SUBA1 \\
\hline CRK1 & AT2G41140 & Encodes CDPK-related kinase 1 (CRK1). & cytosol 0.992 \\
\hline CRK2 & AT3G19100 & Encodes a protein kinase that positively regulates gibberellic acid (GA & cytosol 0.844 , nucleus: 0.129 \\
\hline CRK3 & AT2G46700 & CDPK-related kinase 3 & cytosol 0.971 \\
\hline CRK4 & AT5G24430 & Calcium-dependent protein kinase (CDPK) family protein; & plasma membrane 1.000 \\
\hline CRK5 & AT3G50530 & CDPK-related kinase & plasma membrane 1.000 \\
\hline CRK6 & AT3G49370 & Calcium-dependent protein kinase (CDPK) family protein & plasma membrane 0.773 , cytosol: 0.207 \\
\hline CRK7 & AT3G56760 & Protein kinase superfamily protein & plasma membrane 0.874 , cytosol: 0.125 \\
\hline CRK8 & AT1G49580 & Calcium-dependent protein kinase (CDPK) family protein & cytosol 0.978 \\
\hline
\end{tabular}

Figure S5: Subcellular protein localization database search SUBA (http://suba.live/) for the predicted localization of AtCRK proteins and their annotation.

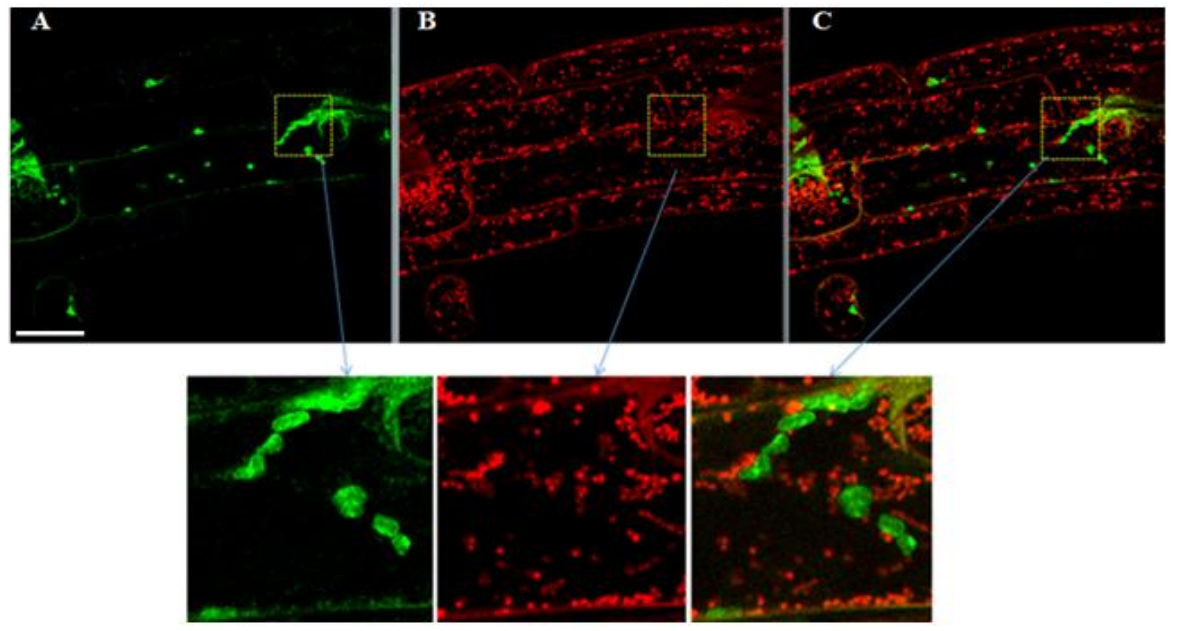

Figure S6: Ruling out the mitochondrial specific marker localization for AtCRK1-eGFP. To rule out the organelle specific localization of AtCRK1-eGFP we tried to check the localization studies with mitochondria specific marker and we noticed that AtCRK1-eGFP does not show any co-localization with MitoTracker Orange dye: GFP fluorescence (A), Mito Tracker Orange (B), merged image(C). The subsets represent enlarged portion of images at specific position (Scale bar: $20 \mu \mathrm{m})$.
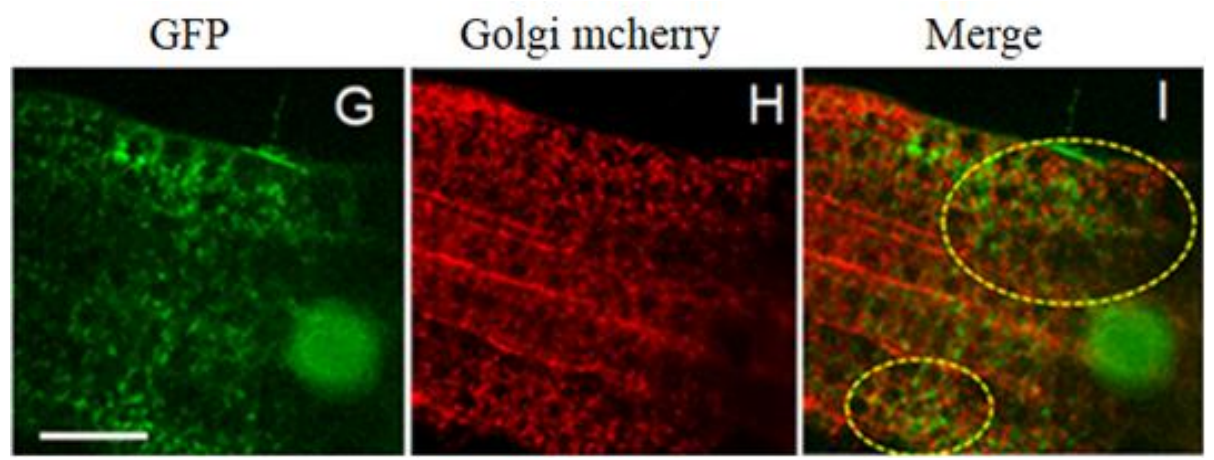

Figure S7: Ruling out the Golgi specific m-cherry marker localization of AtCRK1-GFP. AtCRK1-GFP does not show any co-localization with the Golgi labelled with mCherry: GFP fluorescence $(\mathrm{G})$, mCherry $(\mathrm{H})$, merged (I). Scale bar: $25 \mu \mathrm{m}$ 

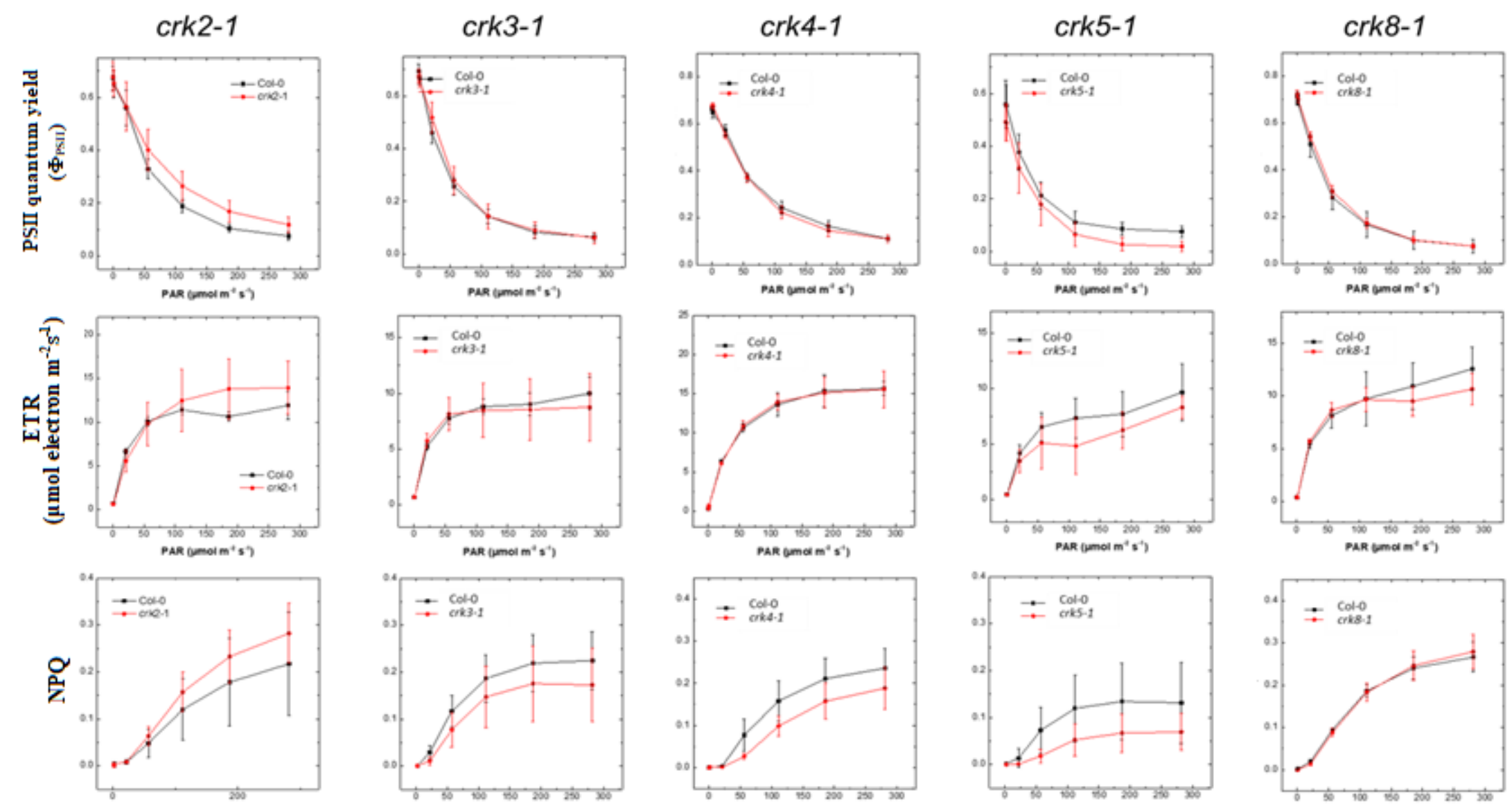

PAR (umol $\left.m^{\prime} s^{\prime}\right)$
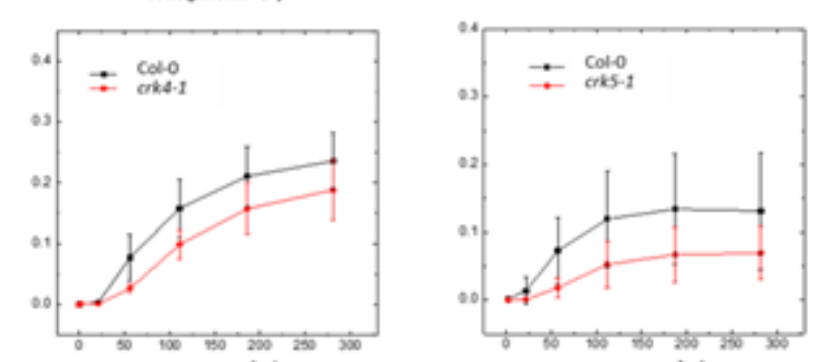

PAR (monol $\left.m^{*} s\right)$

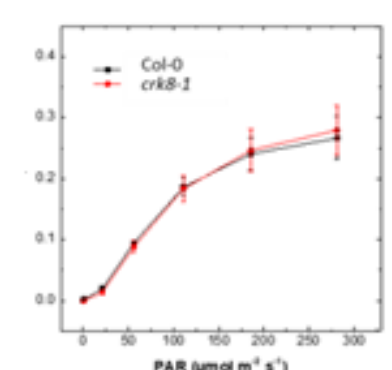

Figure S8: Photosynthetic activities of the five Atcrk mutants in continuous light conditions: Effective PSII quantum yield ( $\left.\Phi_{\mathrm{PSI}}\right)$, Electron transport rates (ETR) and

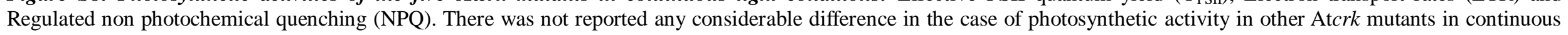
light. 


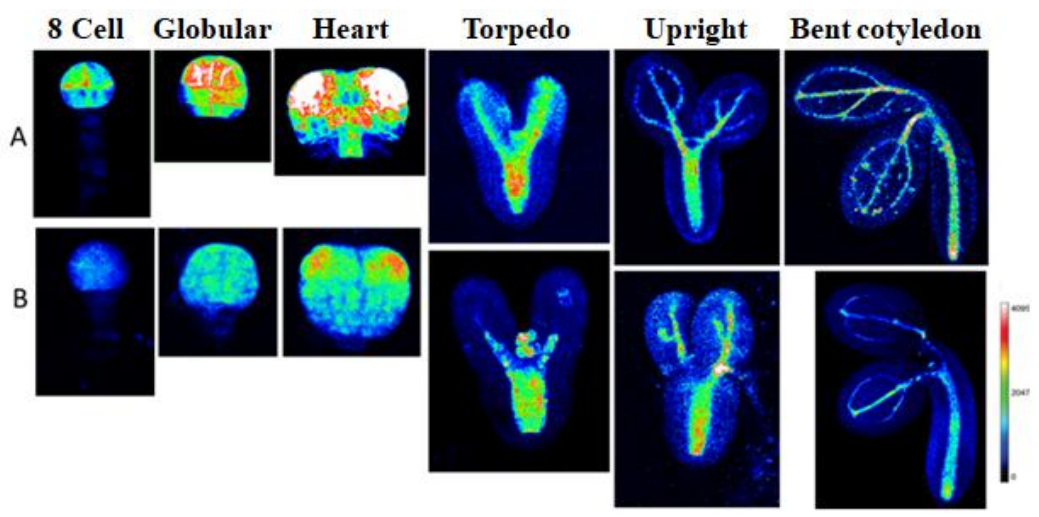

Figure S9: Heat maps of the PIN1-GFP (A) in Col-0 and (B) Atcrk5-1, the signal intensity is proportional with the coloration.

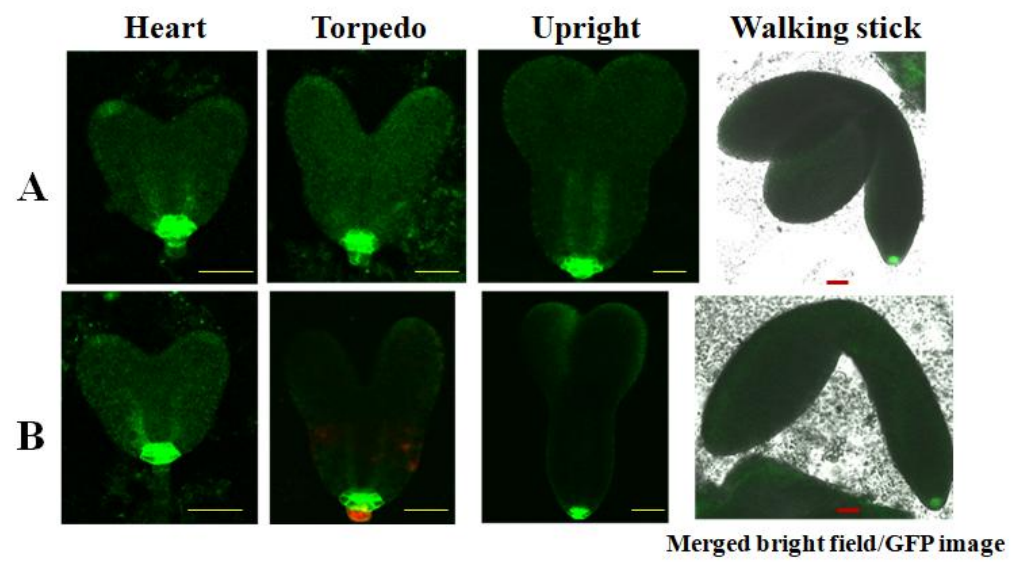

Figure S10: PIN3-GFP abundance during several stages of embryo develoment. (A) During embryo development in wild type (PIN3-GFP in Col-0) and (B) in the mutant (PIN3-GFP in Atcrk5-1). No prominent considerable differences were seen in GFP intensity between them in the investigated stages of embryogenesis. Note merged bright field and GFP image in case of bent cotyledon stage embryo for better visualization of GFP intensity (Scale bars $=100 \mu \mathrm{m})$.
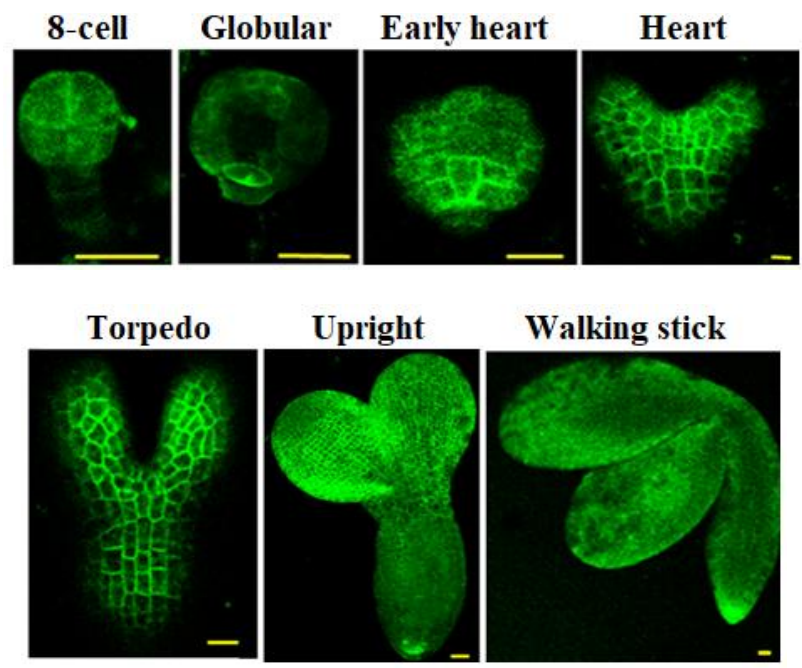

Figure S11: Localization of the AtCRK5-GFP in embryos at several develomental stages. (A) Localization of AtCRK5-GFP in different developing stages of embryo. Note, that AtCRK5 is expressed in all stages of embryo formation. AtCRK5-GFP signal is intensified in the cotyledons and developing root part in upright and walking stick embryo stages. Intracellular localization resembles association to plasma membrane, as it was reported earlier in root (Rigó et al., 2013); (Scale Bars: 8-cell to Heart= $15 \mu \mathrm{m}$; Torpedo to Walking stick= $100 \mu \mathrm{m}$ ). 